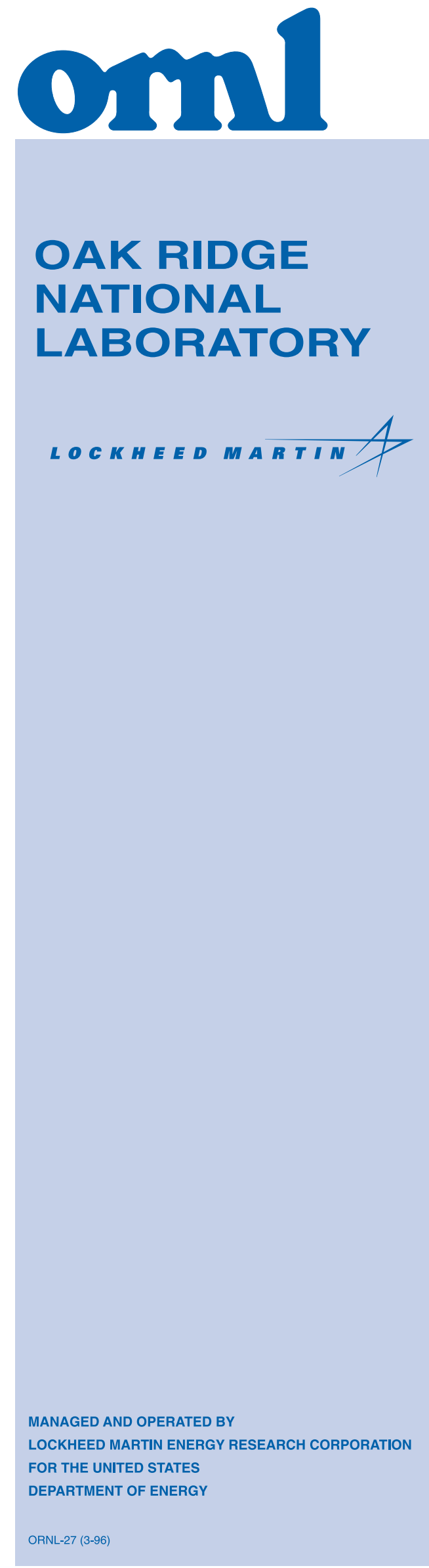

ORNL/TM-1999/207

\title{
Calculational Benchmark Problems for VVER-1000 Mixed Oxide Fuel Cycle
}

\author{
Margaret B. Emmett
}

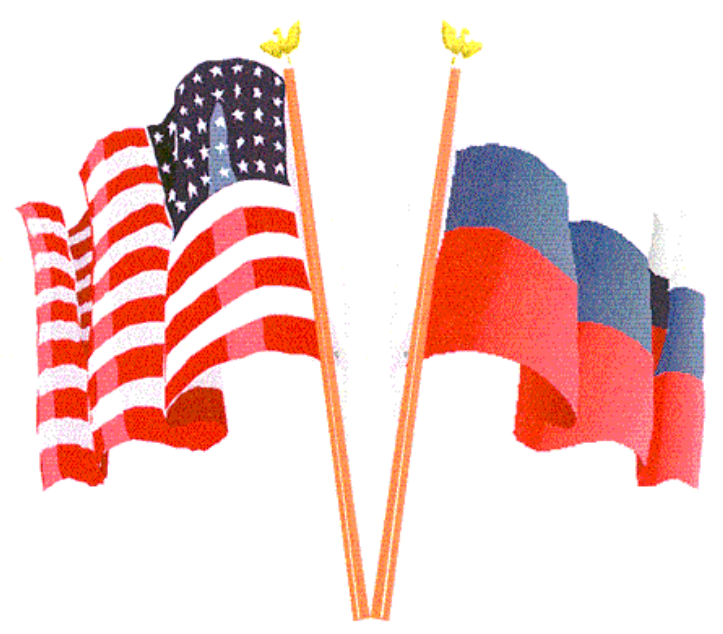

Fissile Materials Disposition Program 
This report has been reproduced from the best available copy.

Reports are available to the public from the following source.

National Technical Information Service

5285 Port Royal Road

Springfield, VA 22161

Telephone 703-605-6000 (1-800-553-6847)

TDD 703-487-4639

Fax 703-605-6900

E-mail orders@ntis.fedworld.gov

Web site http://www.ntis.gov/support

Reports are available to U.S. Department of Energy (DOE) employees, DOE contractors, Energy Technology Data Exchange (ETDE) representatives, and International Nuclear Information System (INIS) representatives from the following source.

Office of Scientific and Technical Information

P.O. Box 62

Oak Ridge, TN 37831

Telephone 865-576-8401

Fax 865-576-5728

E-mail reports@adonis.osti.gov

Web site http://www.osti.gov/products/sources.html

Reports produced after January 1, 1996, are generally available via the DOE Information Bridge.

This report was prepared as an account of work sponsored by an agency of the United States government. Neither the United States government nor any agency thereof, nor any of their employees, makes any warranty, express or implied, or assumes any legal liability or responsibility for the accuracy, completeness, or usefulness of any information, apparatus, product, or process disclosed, or represents that its use would not infringe privately owned rights. Reference herein to any specific commercial product, process, or service by trade name, trademark, manufacturer, or otherwise, does not necessarily constitute or imply its endorsement, recommendation, or favoring by the United States government or any agency thereof. The views and opinions of authors expressed herein do not necessarily state or reflect those of the United States government or any agency thereof. 


\title{
Calculational Benchmark Problems For VVER-1000 Mixed Oxide Fuel Cycle
}

\author{
Margaret B. Emmett
}

Date Published: March 2000

\author{
Prepared by \\ OAK RIDGE NATIONAL LABORATORY \\ P.O. Box 2008 \\ Oak Ridge, Tennessee 37831-6285 \\ managed by \\ LOCKHEED MARTIN ENERGY RESEARCH CORP. \\ for the \\ U.S. DEPARTMENT OF ENERGY \\ under contract DE-AC05-96OR22464
}





\section{CONTENTS}

Page

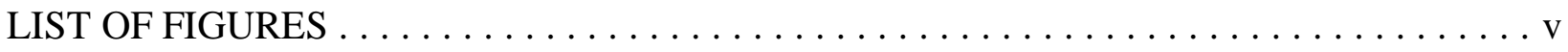

LIST OF TABLES $\ldots \ldots \ldots \ldots \ldots \ldots \ldots \ldots \ldots \ldots \ldots \ldots \ldots$ vii

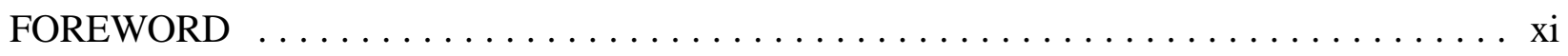

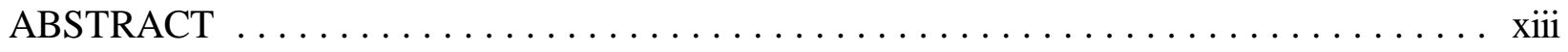

1. INTRODUCTION $\ldots \ldots \ldots \ldots \ldots \ldots \ldots \ldots \ldots \ldots \ldots \ldots \ldots \ldots \ldots$

2. CALCULATIONAL RESULTS FOR TASK I:

CRITICALITY SAFETY STUDY IN STORAGE

3. CALCULATIONAL RESULTS FOR TASK IIa AND IIb:

SHIELDING AND HEAT GENERATION STUDY FOR FRESH FUEL $\ldots \ldots \ldots .7$

4. CALCULATIONAL RESULTS FOR TASK IIc: SHIELDING AND HEAT GENERATION FOR SPENT FUEL

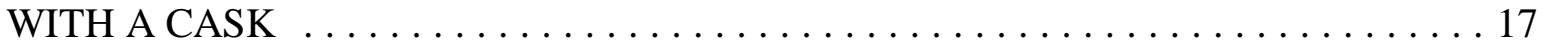

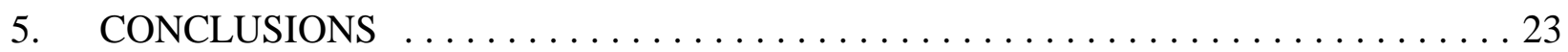

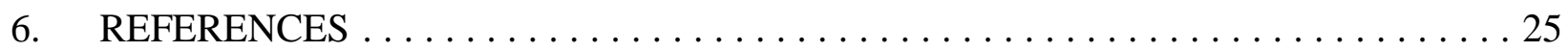

APPENDIX A — DESCRIPTION OF SAFETY ANALYSIS COMPUTATIONAL

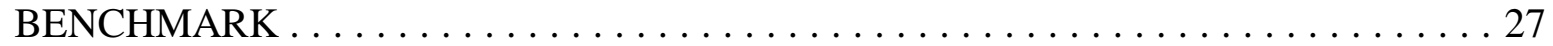

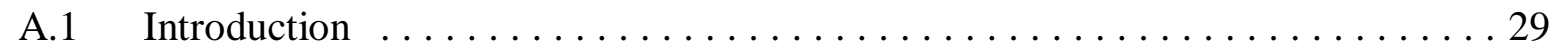

A.2 Task I: Criticality Safety Study in Storage $\ldots \ldots \ldots \ldots \ldots \ldots$

A.3 Task II: Shielding and Heat Generation Study with a Cask . . . . . . . . . . . 30

A.3.1 Task IIa: Study of radioactive characteristics of fresh fuel . . . . . . . 30

A.3.2 Task IIb: Shielding and heat generation study of fresh fuel

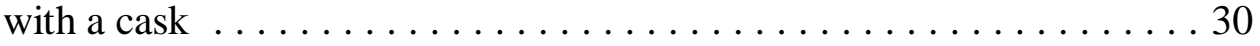

A.3.3 Task IIc: Shielding and heat generation study of spent fuel

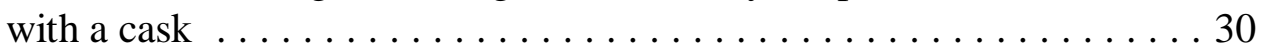

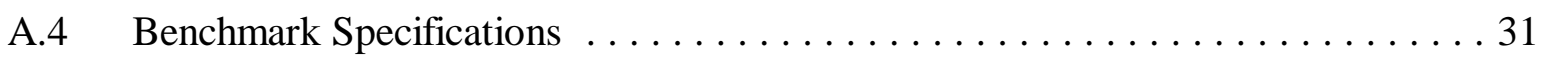

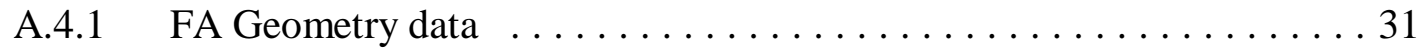

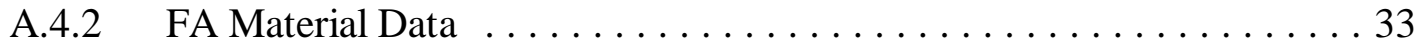

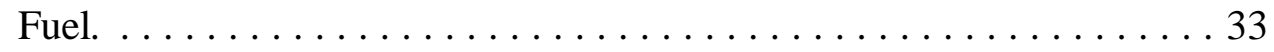

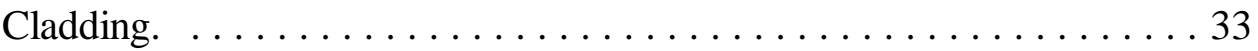

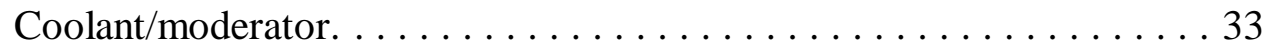




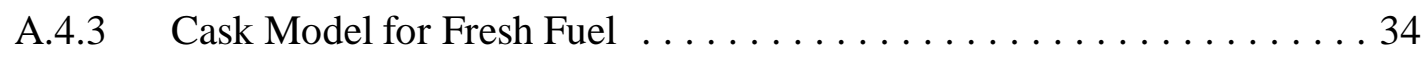

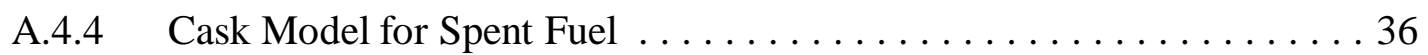

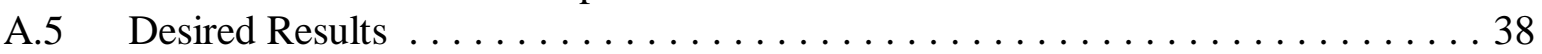

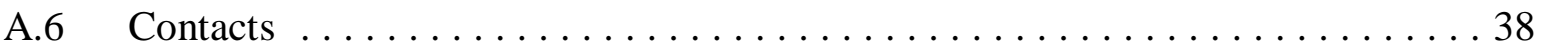

APPENDIX B - CALCULATION RESULTS FOR TASK I:

CRITICALITY SAFETY STUDY IN STORAGE . . . . . . . . . . . . . . 39

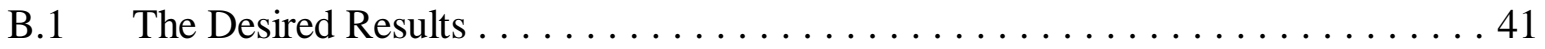

B.2 Short Description of the Used Methods . . . . . . . . . . . . . 43

APPENDIX C - CALCULATION RESULTS FOR TASKS IIa AND IIb . . . . . . . . . 57

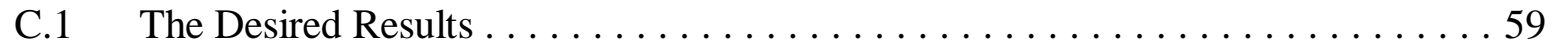

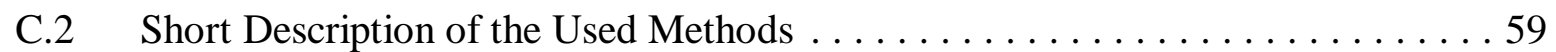

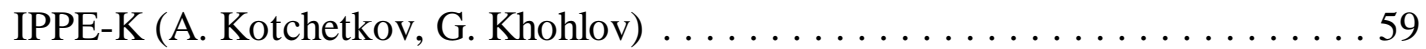

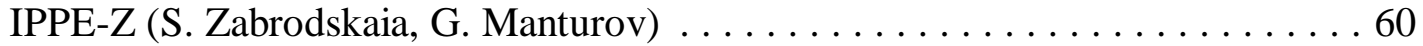

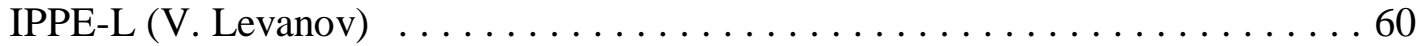

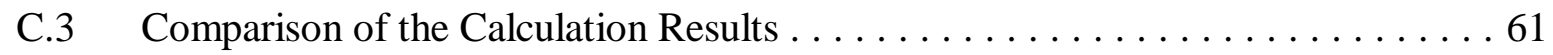

APPENDIX D - CALCULATION RESULTS FOR TASK IIc: SHIELDING AND HEAT

GENERATION STUDY FOR SPENT FUEL WITH A CASK $\ldots \ldots \ldots \ldots \ldots \ldots 77$

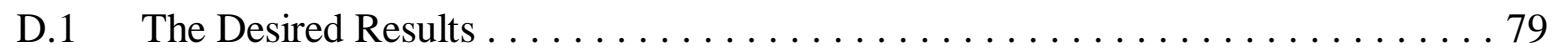

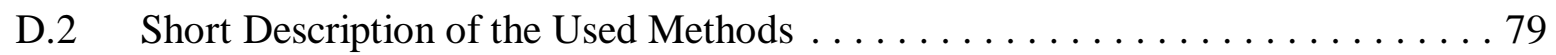

IPPE-K (A. Kotchetkov, G. Khohlov, G.Jerdev) . . . . . . . . . . . . . . . . . . 79

IPPE-Z (S. Zabrodskaia , G. Manturov, A.Tsiboulia) . . . . . . . . . . . . . . 80

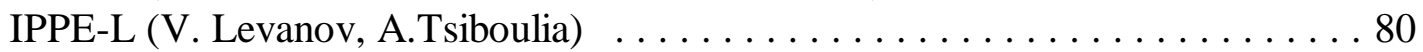

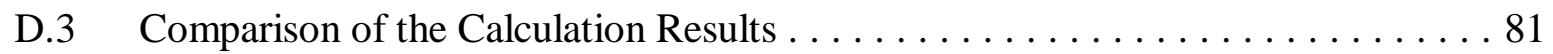

APPENDIX E - RESULTS FOR $K_{\text {eff }}$ WITHOUT WATER POOL $\ldots \ldots \ldots \ldots$ 


\section{LIST OF FIGURES}

Figure

Page

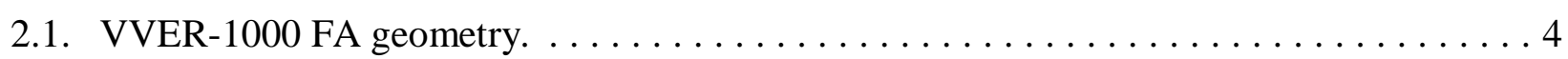

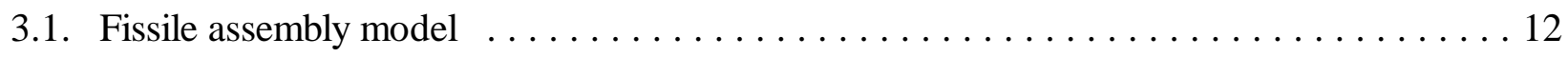

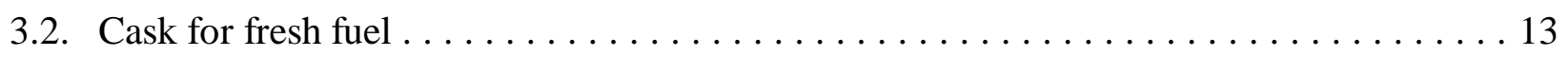

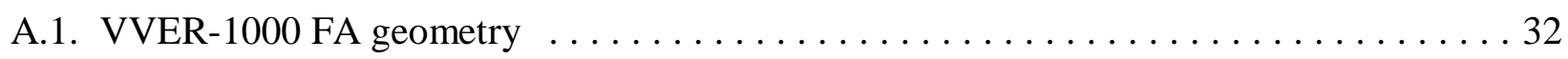

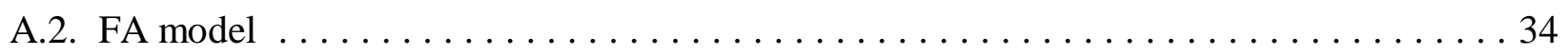

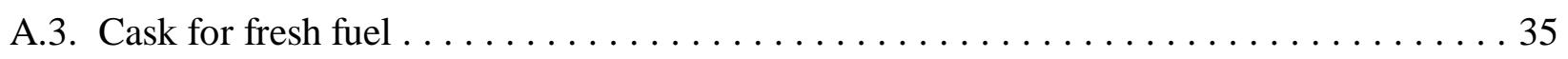

A.4. Cask geometry of spent FA. $\ldots \ldots \ldots \ldots \ldots \ldots \ldots \ldots \ldots \ldots \ldots \ldots \ldots \ldots \ldots \ldots$

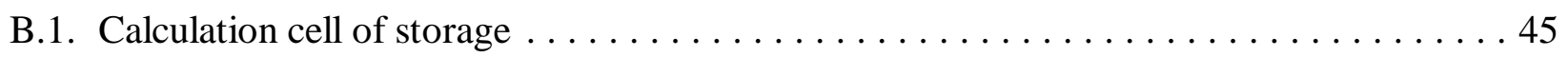

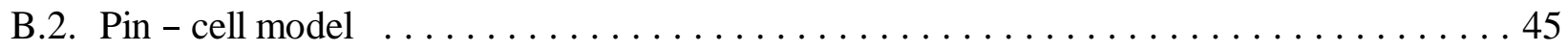

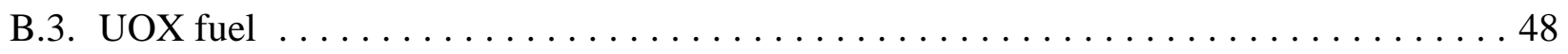

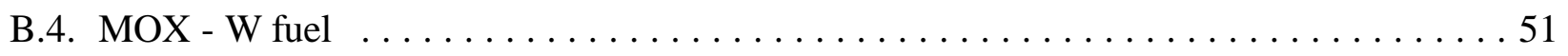

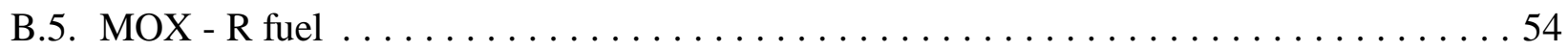

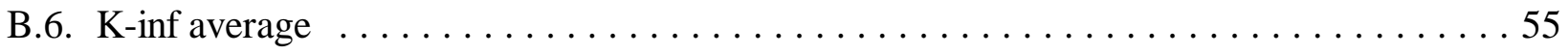





\section{LIST OF TABLES}

Table

Page

2.1. General assembly data $\ldots \ldots \ldots \ldots \ldots \ldots \ldots \ldots \ldots \ldots \ldots \ldots \ldots \ldots$

2.2. MOX and UOX fresh fuel compositions $\ldots \ldots \ldots \ldots \ldots \ldots \ldots \ldots \ldots \ldots$

2.3. $\quad$ K-effective values for fresh fuel for water density study $\ldots \ldots \ldots \ldots \ldots \ldots$

3.1. SAS1 results for UOX fresh fuel single assembly $\ldots \ldots \ldots \ldots \ldots \ldots \ldots \ldots$

3.2. SAS1 results for MOX-W fresh fuel single assembly $\ldots \ldots \ldots \ldots \ldots \ldots \ldots \ldots$

3.3. SAS1 results for MOX-R fresh fuel single assembly $\ldots \ldots \ldots \ldots \ldots \ldots \ldots \ldots$

3.4. Total (alpha-n + spon. fission) neutron source spectrum $\ldots \ldots \ldots \ldots \ldots \ldots$

3.5. Neutron source intensity for fresh fuel $\ldots \ldots \ldots \ldots \ldots \ldots \ldots \ldots \ldots \ldots$

3.6. Gamma source spectrum for fresh fuel $\ldots \ldots \ldots \ldots \ldots \ldots \ldots \ldots \ldots$

3.7. Total neutron and gamma source from SAS2 $\ldots \ldots \ldots \ldots \ldots \ldots \ldots \ldots$

3.8. Masses of actinides for a fissile assembly of fresh fuel $\ldots \ldots \ldots \ldots \ldots \ldots \ldots$

3.9. Atom composition of cylinder model of FA in the cask for fresh fuel $\ldots \ldots \ldots \ldots$

3.10. Atom composition of structure materials in the cask for fresh fuel $\ldots \ldots \ldots \ldots$

3.11. SAS2 results for UOX fresh fuel $\ldots \ldots \ldots \ldots \ldots \ldots \ldots \ldots \ldots \ldots$

3.12. SAS2 results for MOX-W fresh fuel $\ldots \ldots \ldots \ldots \ldots \ldots \ldots \ldots \ldots$

3.13. SAS2 results for MOX-R fresh fuel $\ldots \ldots \ldots \ldots \ldots \ldots \ldots \ldots \ldots \ldots$

3.14. Heat generation for fresh fuel cask $\ldots \ldots \ldots \ldots \ldots \ldots \ldots \ldots \ldots \ldots \ldots$

4.1. Borated-water composition $\ldots \ldots \ldots \ldots \ldots \ldots \ldots \ldots \ldots \ldots \ldots \ldots \ldots \ldots$

4.2. Atom composition of structure materials in the cask for spent fuel $\ldots \ldots \ldots \ldots$

4.3. $\quad$ SAS2 results for UOX spent fuel $\ldots \ldots \ldots \ldots \ldots \ldots \ldots \ldots \ldots \ldots \ldots \ldots$

4.4. $\quad$ SAS2 results for MOX-W spent fuel $\ldots \ldots \ldots \ldots \ldots \ldots \ldots \ldots \ldots \ldots$ 
4.5. $\quad$ SAS2 results for MOX-R spent fuel $\ldots \ldots \ldots \ldots \ldots \ldots \ldots \ldots \ldots \ldots \ldots \ldots \ldots \ldots \ldots \ldots \ldots$

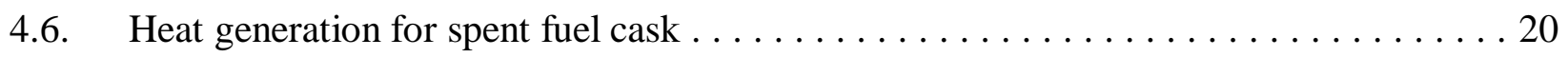

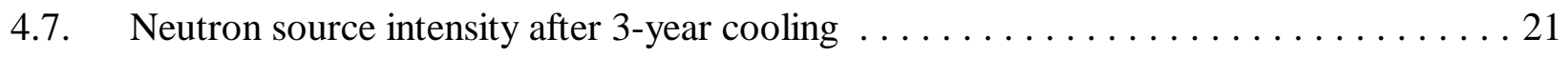

4.8. $\quad$ Total neutron and gamma source from SAS2 $\ldots \ldots \ldots \ldots \ldots \ldots \ldots \ldots \ldots \ldots \ldots \ldots$

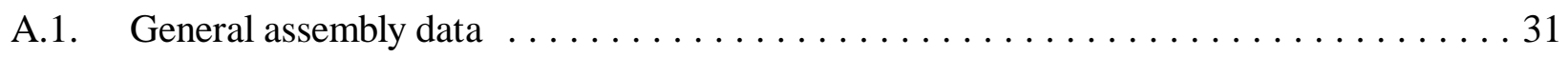

A.2. MOX and UOX fresh fuel compositions $\ldots \ldots \ldots \ldots \ldots \ldots \ldots \ldots \ldots \ldots \ldots \ldots \ldots \ldots \ldots \ldots$

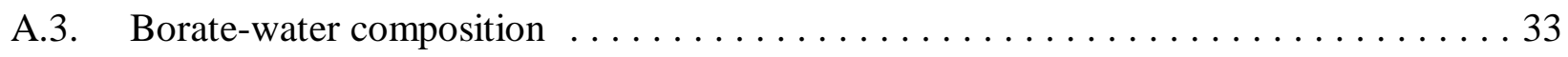

A.4. Atom composition of cylinder model of FA in the cask for fresh fuel ........... 34

A.5. Atom composition of structure materials in the cask for fresh fuel $\ldots \ldots \ldots \ldots 35$

A.6. Atom composition of structure materials in the cask for spent fuel . .......... 36

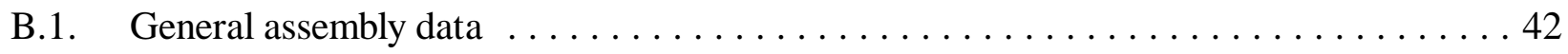

B.2. $\quad$ MOX and UOX fresh fuel compositions $\ldots \ldots \ldots \ldots \ldots \ldots \ldots \ldots \ldots \ldots$

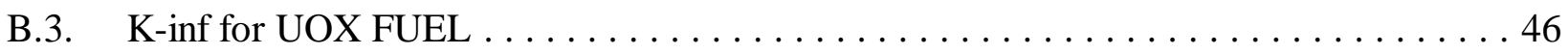

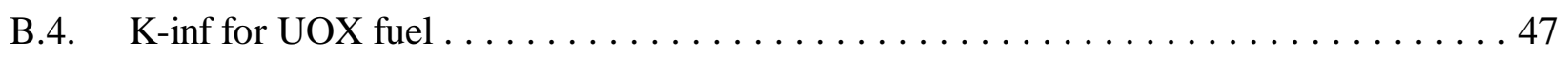

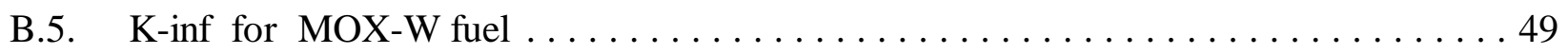

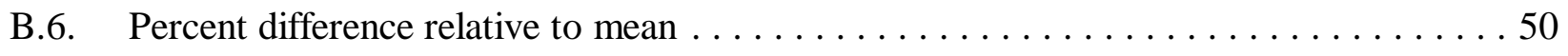

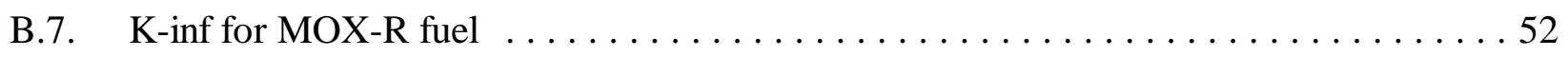

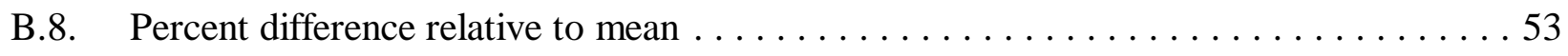

C.1. UO2 - Comparison of dose rates $[\mathrm{mSv} / \mathrm{h}]$ calculated by different methods $\ldots \ldots \ldots 61$

C.2. MOX WEAPON - Comparison of dose rates $[\mathrm{mSv} / \mathrm{h}]$ calculated by different methods . 62

C.3. MOX REACTOR - Comparison of dose rates [mSv/h] calculated by different methods 63

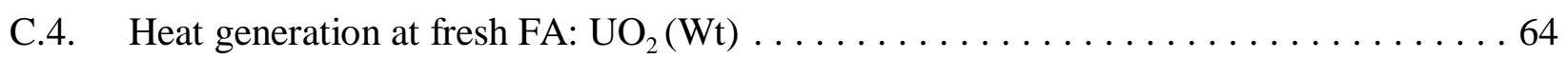

C.5. $\quad$ Heat generation at fresh FA: MOX-W $(4.2 \%)(\mathrm{Wt}) \ldots \ldots \ldots \ldots \ldots \ldots$

C.6. Heat generation at fresh FA: MOX-R (6.1\%) (Wt) $\ldots \ldots \ldots \ldots \ldots \ldots \ldots \ldots$ 
C.7. Comparison of main radioactive characteristics: $\mathrm{T}_{1 / 2}$ (year) $\ldots \ldots \ldots \ldots \ldots \ldots 6$

C.8. Comparison of main radioactive characteristics: Decay energy (MeV) and number of $\alpha$-particles per decay . ........................ 67

C.9. Comparison of main radioactive characteristics: yield of reaction $(\alpha-n)$

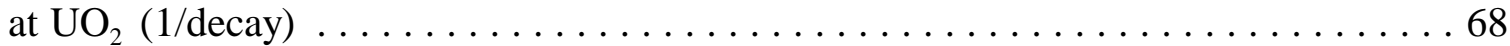

C.10. Comparison of main radioactive characteristics: spontaneous fission (1/decay) . . . . 69

C.11. Masses of actinides at $1 \mathrm{FA}$ of fresh fuel $\mathrm{UO}_{2}(\mathrm{~g}) \ldots \ldots \ldots \ldots \ldots \ldots \ldots \ldots \ldots$

C.12. Masses of actinides at $1 \mathrm{FA}$ of fresh fuel MOX-W (4.2\%) (g) $\ldots \ldots \ldots \ldots \ldots 70$

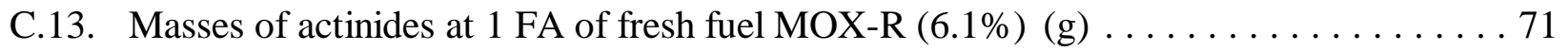

C.14. Neutron source strength at $1 \mathrm{FA}$ of fresh fuel $\mathrm{UO}_{2}(\mathrm{n} / \mathrm{s}) \ldots \ldots \ldots \ldots \ldots \ldots \ldots$.

C.15. Neutron source strength at 1 FA of fresh fuel MOX-W $(4.2 \%)(\mathrm{n} / \mathrm{s}) \ldots \ldots \ldots \ldots \ldots 72$

C.16. Neutron source strength at 1 FA of fresh fuel MOX-R (6.1\%) $(\mathrm{n} / \mathrm{s}) \ldots \ldots \ldots \ldots 72$

C.17. Neutron source spectrum normalized to 1 and total intensity at 1 FA calculated by IPPE-Z using ORIGEN $(\mathrm{n} / \mathrm{s}) \ldots \ldots \ldots \ldots \ldots \ldots \ldots \ldots \ldots \ldots \ldots$

C.18. Gamma source spectrum normalized to 1 and total intensity at $1 \mathrm{FA}$ calculated by IPPE-Z (photons/s) … . . . . . . . . . . . . . . . . . 73

C.19. Neutron source spectrum normalized to 1 and total intensity at $1 \mathrm{FA}$

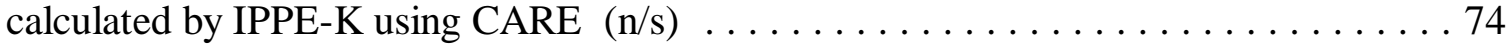

C.20. Gamma source spectrum normalized to 1 and total intensity at $1 \mathrm{FA}$ calculated by IPPE-K for storage time $\mathrm{t}=0$ (photons $/ \mathrm{s}) \ldots \ldots \ldots \ldots \ldots \ldots \ldots$

C.21. Gamma source spectrum normalized to 1 and total intensity at $1 \mathrm{FA}$ calculated by IPPE-K for storage time $\mathrm{t}=10$ years (photons/s) $\ldots \ldots \ldots \ldots \ldots 75$

D.1. $\mathrm{UO}_{2}$ - Comparison of dose rates $[\mathrm{mSv} / \mathrm{h}]$ calculated by different methods $\ldots \ldots \ldots$. 81

D.2. MOX WEAPON - Comparison of dose rates [mSv/h] calculated by different methods . 82

D.3. MOX REACTOR - Comparison of dose rates [mSv/h] calculated by different methods 83

D.4. The masses of actinides in one irradiated FA(g) after 3 years cooling time UOX .................................... 84 
D.5. The masses of actinides in one irradiated $\mathrm{FA}(\mathrm{g})$ after 3 years cooling time MOX (weapon) ................................... 85

D.6. The masses of actinides in one irradiated $\mathrm{FA}(\mathrm{g})$ after 3 years cooling time MOX (reactor) ....................................... . 86

D.7. Heat generation via cooling time for 1 irradiated FA, $\mathrm{Wt} \ldots \ldots \ldots \ldots \ldots \ldots$. $\ldots \ldots$ 


\section{FOREWORD}

This document fulfils milestone 10.2.2.2e in the Fissile Materials Disposition Program Annual Operations Plan for 1999. This report contains the final computational results for the United States. The Russian results which are provided in this report are for the convenience of the reader and are not official documentation. 



\title{
Calculational Benchmark Problems for VVER-1000 Mixed Oxide Fuel Cycle
}

\author{
Margaret B. Emmett
}

\begin{abstract}
Standard problems were created to test the ability of American and Russian computational methods and data regarding the analysis of the storage and handling of Russian pressurized water reactor (VVER) mixed oxide fuel. Criticality safety and radiation shielding problems were analyzed. Analysis of American and Russian multiplication factors for fresh fuel storage for low-enriched uranium (UOX), weapons- (MOX-W) and reactor-grade (MOX-R) MOX differ by less than $2 \%$ for all variations of water density. For shielding calculations for fresh fuel, the ORNL results for the neutron source differ from the Russian results by less than $1 \%$ for UOX and MOX-R and by approximately $3 \%$ for MOX-W. For shielding calculations for fresh fuel assemblies, neutron dose rates at the surface of the assemblies differ from the Russian results by 5\% to $9 \%$; the level of agreement for gamma dose varies depending on the type of fuel, with UOX differing by the largest amount. The use of different gamma group structures and instantaneous versus asymptotic decay assumptions also complicate the comparison. For the calculation of dose rates from spent fuel in a shipping cask, the neutron source for UOX after 3-year cooling is within $1 \%$ and for MOX-W within $5 \%$ of one of the Russian results while the MOX-R difference is the largest at over $10 \%$. These studies are a portion of the documentation required by the Russian nuclear regulatory authority, GAN, in order to certify Russian programs and data as being acceptably accurate for the analysis of mixed oxide fuels.
\end{abstract}





\section{INTRODUCTION}

A goal of the Fissile Materials Disposition Program is to irradiate Russian, weapons-usable plutonium in Russian pressurized water reactors (VVERs). The fuel cycle which must be developed to support the irradiations services will include fresh fuel shipment to the reactor sites, fresh fuel storage at the reactor sites and transportation of spent, mixed oxide (MOX) fuel from the reactors to ultimate disposal. Assessment of the nuclear safety of these procedures will likely require the use of computational methods. The calculation of computational benchmarks (standard problems) is an accepted method of verifying computational methods.

Specifications were provided jointly by the Russian and American participants in the Fissile Materials Disposition Program (FMDP) for a calculational benchmark problem set for fissile material disposition with a VVER-type reactor. Appendix A contains the specifications. The study used the following fuels: mixed oxide (MOX) with weapons-grade plutonium, MOX consisting of civil plutonium fuel (reactor-grade) and the traditional uranium dioxide (UOX) low enriched fuel. Task I was a study of criticality safety in fresh fuel storage for the three types of fuel. Task II is a three-part task studying the shielding and radioactive characteristics when the fissile assembly is transported. Task IIa is a study of the radioactive characteristics of a fissile assembly of fresh fuel without a container. Task IIb is a study of a fissile assembly of fresh fuel within a cask. Task IIc is a study of a fissile assembly with a spent fuel cask. The cask model is typical of those used to transport fissile assemblies of spent fuel. Appendices B-D contain Russian computational results for these Tasks. 



\section{CALCULATIONAL RESULTS FOR TASK I: CRITICALITY SAFETY STUDY IN STORAGE}

Task I was a study of criticality safety in fresh fuel storage for three types of fuel. The geometry specifications for the fissile assembly are given in Table 2.1. The model was typical of a VVER-1000 in an assembly lattice of 312 fuel pins with 18 control rod guide tubes, and a central instrumentation channel. The pool storage model is an infinite lattice with a pitch of $40 \mathrm{~cm}$. Figure 2.1 illustrates the geometry with reflected boundaries on the 6 outermost surfaces. Table 2.2 contains the fresh fuel compositions of the UOX and MOX in units of atoms $/ \mathrm{barn}-\mathrm{cm}$. The cladding is a Zirconium composition with an atom density 0.0423 atoms/barn-cm. This calculation is a parameter study of the change in $k_{\text {eff }}$ due to changes in the water density. The infinite pool storage calculation contains variable density water at $\mathrm{T}=300 \mathrm{~K}$. The $\mathrm{H}_{2} \mathrm{O}$ densities, $\gamma\left(\mathrm{H}_{2} \mathrm{O}\right)$, were 1.0, 0.9, $0.8,0.7,0.6,0.5,0.4,0.3,0.2,0.1,0.05,0.03,0.02$, and $0\left(\mathrm{~g} / \mathrm{cm}^{3}\right)$, where for $\gamma\left(\mathrm{H}_{2} \mathrm{O}\right)=1 \mathrm{~g} / \mathrm{cm}^{3}$, the densities in atoms/(barn-cm) are $\mathrm{H}=.06694$ and $\mathrm{O}=0.03347$. The cross-section library used was the SCALE $^{1}$ 238-group library based on ENDF/B-V. The assembly was modeled in KENO-VI geometry, using mirror reflection as the boundary condition. The results are given in Table 2.3. As the moderator density approaches unity, the single-unit $k_{\text {eff }}$ is approached; e.g., for UOX the singleunit $k_{\text {eff }}$ was calculated to be 0.8858 . In other words, for $\gamma\left(\mathrm{H}_{2} \mathrm{O}\right)=1$ down to $\gamma\left(\mathrm{H}_{2} \mathrm{O}\right)=0.7$, the assembly acts like a single unit because there is enough water in the system to isolate the assemblies from each other. The reactivity decreases up to the point where the assemblies are no longer isolated; and then, it increases until optimal moderation is reached. At that point (approximately $0.1 \mathrm{~g} / \mathrm{cm}^{3}$ ), the $k_{\text {eff }}$ starts to drop off because the assemblies are undermoderated. All cases were run using KENO-VI from the SCALE system for a total of 500,000 particles. The SCALE4.3R version of CSAS6 which processes the cross-section data and executes KENO-VI was used. Appendix E has some additional comments on calculation of the $k_{\text {eff }}$ for the dry case. 
Table 2.1. General assembly data

\begin{tabular}{lc}
\hline \multicolumn{1}{c}{ Parameter } & Value \\
\hline Fuel pins & 312 \\
Number of fuel pins & 18 \\
Number of guide tubes & 1 \\
Number of instrumentation tubes & 1.275 \\
Pin pitch, cm & \\
Fuel rods & 0.772 \\
Pellet diameter, cm & 0.772 \\
Clad inside diameter, cm & 0.910 \\
Clad outside diameter, cm & $\mathrm{Zr}$ \\
Clad material & 353.0 \\
Active fuel length, cm & \\
Guide tubes & 1.090 \\
Inside diameter, cm & 1.265 \\
Outside diameter, cm & $\mathrm{Zr}$ \\
Material & \\
Central instrumentation tube & 0.960 \\
Inside diameter, cm & 1.125 \\
Outside diameter, cm & $\mathrm{Zr}$ \\
Material & \\
\hline
\end{tabular}

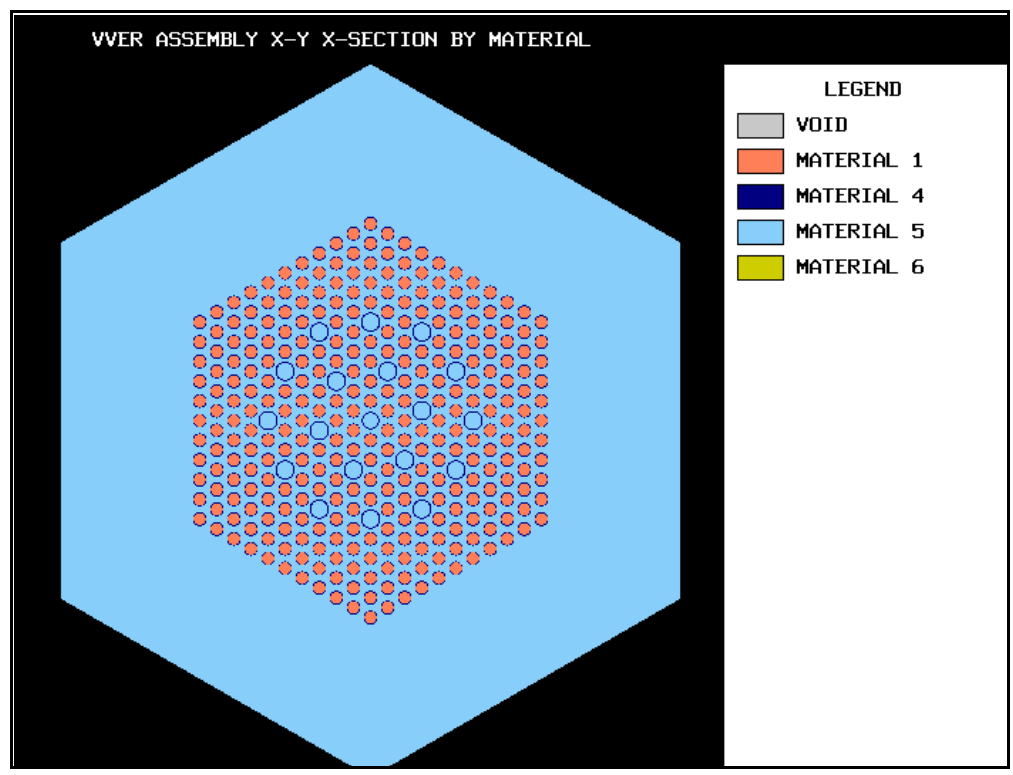

Fig. 2.1. VVER-1000 FA geometry. 
Table 2.2. MOX and UOX fresh fuel compositions [atoms/(b cm)]

\begin{tabular}{cccr}
\hline Nuclides & MOX-W $(4.2 \%)$ & MOX-R $(6.1 \%)$ & UOX (4.4\%) \\
\hline${ }^{16} \mathrm{O}$ & $4.3036 \times 10^{-2}$ & $4.3051 \times 10^{-2}$ & $3.9235 \times 10^{-2}$ \\
${ }^{234} \mathrm{U}$ & $<2.0 \times 10^{-7}$ & $<2.0 \times 10^{-7}$ & $8.0 \times 10^{-6}$ \\
${ }^{235} \mathrm{U}$ & $4.1762 \times 10^{-5}$ & $4.0964 \times 10^{-5}$ & $8.7370 \times 10^{-4}$ \\
${ }^{238} \mathrm{U}$ & $2.0576 \times 10^{-2}$ & $2.0183 \times 10^{-5}$ & $1.8744 \times 10^{-2}$ \\
${ }^{236} \mathrm{Pu}$ & $<1.0 \times 10^{-12}$ & $<1.0 \times 10^{-10}$ & \\
${ }^{238} \mathrm{Pu}$ & $1.8089 \times 10^{-7}$ & $1.9720 \times 10^{-5}$ & \\
${ }^{239} \mathrm{Pu}$ & $8.4610 \times 10^{-4}$ & $7.5671 \times 10^{-4}$ & \\
${ }^{240} \mathrm{Pu}$ & $5.2111 \times 10^{-5}$ & $3.1941 \times 10^{-4}$ & \\
${ }^{241} \mathrm{Pu}$ & $1.6078 \times 10^{-6}$ & $1.2464 \times 10^{-4}$ & \\
${ }^{242} \mathrm{Pu}$ & $2.6685 \times 10^{-7}$ & $6.8527 \times 10^{-5}$ & \\
${ }^{241} \mathrm{Am}$ & $1.7864 \times 10^{-7}$ & $1.6878 \times 10^{-5}$ & \\
\hline
\end{tabular}

Table 2.3. K-effective values for fresh fuel for water density study

\begin{tabular}{cccccc}
\hline $\begin{array}{c}\mathrm{H}_{2} \mathrm{O} \text { Density } \\
\mathrm{g} / \mathrm{cc}\end{array}$ & $\begin{array}{c}\text { Hydrogen } \\
\text { (atoms/b-cm) }\end{array}$ & $\begin{array}{c}\text { Oxygen } \\
\text { (atoms/b-cm) }\end{array}$ & $\mathrm{UO}_{2}$ & MOX-W & MOX-R \\
\hline 1.0 & $6.6940 \times 10^{-2}$ & $3.3470 \times 10^{-2}$ & 0.9254 & 0.9195 & 0.8330 \\
0.9 & $6.0246 \times 10^{-2}$ & $3.0123 \times 10^{-2}$ & 0.9031 & 0.8984 & 0.8125 \\
0.8 & $5.3552 \times 10^{-2}$ & $2.6776 \times 10^{-2}$ & 0.8853 & 0.8798 & 0.8004 \\
0.7 & $4.6858 \times 10^{-2}$ & $2.3429 \times 10^{-2}$ & 0.8795 & 0.8787 & 0.8011 \\
0.6 & $4.0164 \times 10^{-2}$ & $2.0082 \times 10^{-2}$ & 0.8916 & 0.8949 & 0.8176 \\
0.5 & $3.3470 \times 10^{-2}$ & $1.6735 \times 10^{-2}$ & 0.9325 & 0.9373 & 0.8608 \\
0.4 & $2.6776 \times 10^{-2}$ & $1.3388 \times 10^{-2}$ & 1.014 & 1.020 & 0.9404 \\
0.3 & $2.0082 \times 10^{-2}$ & $1.0041 \times 10^{-2}$ & 1.135 & 1.139 & 1.051 \\
0.2 & $1.3388 \times 10^{-2}$ & $6.6940 \times 10^{-3}$ & 1.269 & 1.258 & 1.158 \\
0.1 & $6.6940 \times 10^{-3}$ & $3.3470 \times 10^{-3}$ & 1.287 & 1.238 & 1.136 \\
0.05 & $3.3470 \times 10^{-3}$ & $1.6735 \times 10^{-3}$ & 1.098 & 1.042 & 0.9710 \\
0.03 & $2.0082 \times 10^{-3}$ & $1.0041 \times 10^{-3}$ & 0.9130 & 0.8774 & 0.8460 \\
0.02 & $1.3388 \times 10^{-3}$ & $6.6940 \times 10^{-4}$ & 0.7830 & 0.7714 & 0.7689 \\
\hline $0($ dry $)$ & 0 & 0 & 0.4594 & 0.5244 & 0.5707 \\
\hline
\end{tabular}

*All results are from SCALE 4.3R version of KENO-VI for 250 generations of 2000 particles using the 238 Group Library. 


\section{CALCULATIONAL RESULTS FOR TASK IIa AND IIb: SHIELDING AND HEAT GENERATION STUDY FOR FRESH FUEL}

Task IIa is a study of the radioactive characteristics of fresh fuel in a fissile assembly without a container (cask). A dry assembly of fresh fuel with geometry specifications from Table 2.1 and fresh fuel compositions from Table 2.2 was used. The temperature of the fissile assembly was $300 \mathrm{~K}$.

For Task IIa, calculations of the dose rates at the surface of the assembly and at $0.5,1$, and 2 meters from this surface were calculated using the one-dimensional SCALE module SAS1. The dose rate and flux results for all three types of fuel are given in Tables 3.1-3.3.

Table 3.1. SAS1 results for UOX fresh fuel single assembly

\begin{tabular}{lcccc}
\hline \multirow{2}{*}{ Detector } & $\begin{array}{c}\text { Flux } \\
\left(\mathrm{n} / \mathrm{cm}^{2} / \mathrm{sec}\right)\end{array}$ & $\begin{array}{c}\text { Dose } \\
(\mathrm{rem} / \mathrm{hr})\end{array}$ & $\begin{array}{c}\text { Flux } \\
\left(\mathrm{photons} / \mathrm{cm}^{2} / \mathrm{sec}\right)\end{array}$ & $\begin{array}{c}\text { Dose } \\
(\mathrm{rem} / \mathrm{hr})\end{array}$ \\
\cline { 2 - 5 } & $3.735 \times 10^{-1}$ & $4.252 \times 10^{-5}$ & $5.184 \times 10^{2}$ & $2.233 \times 10^{-4}$ \\
At surface & $5.328 \times 10^{-2}$ & $6.064 \times 10^{-6}$ & $7.294 \times 10^{1}$ & $3.124 \times 10^{-5}$ \\
$0.5 \mathrm{~m}$ from surface & $2.649 \times 10^{-2}$ & $3.008 \times 10^{-6}$ & $3.723 \times 10^{1}$ & $1.592 \times 10^{-5}$ \\
$1 \mathrm{~m}$ from surface & $1.047 \times 10^{-2}$ & $1.185 \times 10^{-6}$ & $1.530 \times 10^{1}$ & $6.533 \times 10^{-6}$ \\
\hline
\end{tabular}

Table 3.2. SAS1 results for MOX-W fresh fuel single assembly

\begin{tabular}{lcccc}
\hline \multirow{2}{*}{ Detector } & \multicolumn{2}{c}{ Neutron } & \multicolumn{2}{c}{ Gamma } \\
\cline { 2 - 5 } & $\begin{array}{c}\text { Flux } \\
\left(\mathrm{n} / \mathrm{cm}^{2} / \mathrm{sec}\right)\end{array}$ & $\begin{array}{c}\text { Dose } \\
(\mathrm{rem} / \mathrm{hr})\end{array}$ & $\begin{array}{c}\text { Flux } \\
\left(\mathrm{photons} / \mathrm{cm}^{2} / \mathrm{sec}\right)\end{array}$ & $\begin{array}{c}\text { Dose } \\
(\mathrm{rem} / \mathrm{hr})\end{array}$ \\
\hline At surface & $1.393 \times 10^{2}$ & $1.602 \times 10^{-2}$ & $1.552 \times 10^{4}$ & $1.115 \times 10^{-2}$ \\
$0.5 \mathrm{~m}$ from surface & $1.989 \times 10^{1}$ & $2.286 \times 10^{-3}$ & $2.217 \times 10^{3}$ & $1.522 \times 10^{-3}$ \\
$1 \mathrm{~m}$ from surface & $9.895 \times 10^{0}$ & $1.135 \times 10^{-3}$ & $1.144 \times 10^{3}$ & $7.725 \times 10^{-4}$ \\
$2 \mathrm{~m}$ from surface & $3.917 \times 10^{0}$ & $4.479 \times 10^{-4}$ & $4.815 \times 10^{2}$ & $3.173 \times 10^{-4}$ \\
\hline
\end{tabular}


Table 3.3. SAS1 results for MOX-R fresh fuel single assembly

\begin{tabular}{lcccc}
\hline \multirow{2}{*}{ Detector } & \multicolumn{2}{c}{ Neutron } & \multicolumn{2}{c}{ Gamma } \\
\cline { 2 - 5 } & $\begin{array}{c}\text { Flux } \\
\left(\mathrm{n} / \mathrm{cm}^{2} / \mathrm{sec}\right)\end{array}$ & $\begin{array}{c}\text { Dose } \\
(\mathrm{rem} / \mathrm{hr})\end{array}$ & $\begin{array}{c}\text { Flux } \\
\left(\mathrm{photons} / \mathrm{cm}^{2} / \mathrm{sec}\right)\end{array}$ & $\begin{array}{c}\text { Dose } \\
(\mathrm{rem} / \mathrm{hr})\end{array}$ \\
\hline At surface & $1.278 \times 10^{3}$ & $1.470 \times 10^{-1}$ & $4.321 \times 10^{5}$ & $1.304 \times 10^{-1}$ \\
$0.5 \mathrm{~m}$ from surface & $1.825 \times 10^{2}$ & $2.097 \times 10^{-2}$ & $7.134 \times 10^{4}$ & $2.106 \times 10^{-2}$ \\
$1 \mathrm{~m}$ from surface & $9.081 \times 10^{1}$ & $1.041 \times 10^{-2}$ & $3.839 \times 10^{4}$ & $1.126 \times 10^{-2}$ \\
$2 \mathrm{~m}$ from surface & $3.595 \times 10^{1}$ & $4.109 \times 10^{-3}$ & $1.719 \times 10^{4}$ & $4.998 \times 10^{-3}$ \\
\hline
\end{tabular}

The neutron and gamma source strengths were calculated using the SAS2 code sequence. Table 3.4 gives the total neutron source spectra in the SCALE 27 neutron-group structure for a single assembly for each of the three fuel types. The neutron source intensity by separate isotope is given in Table 3.5. The gamma source spectra is contained in Table 3.6 in the 18-gamma-group structure from the SCALE 27n-18 gamma-group library. This library is based on data from the Evaluated Nuclear Data File/B, Version IV (ENDF/B-IV). In order to make it easier to compare the data, the total neutron and gamma sources for UOX, MOX-R and MOX-W are presented in Table 3.7.

For the purpose of confirming the SAS2 results, an additional calculation was done using the PC code ORIGEN-ARP? The results were comparable. In order to use ORIGEN-ARP, the masses of the nuclides in the three types of fuel were calculated and used as input; Table 3.8 contains the calculated masses. In each case, all sources were generated corresponding to minimal decay; therefore, no equilibrium assumptions were made for daughter products. 
Table 3.4. Total (alpha-n + spon. fission) neutron source spectrum

\begin{tabular}{|c|c|c|c|c|}
\hline & & \multicolumn{3}{|c|}{ Neutrons/sec } \\
\hline & Boundaries, $\mathrm{MeV}$ & UOX & MOX-R & MOX-W \\
\hline 1 & $6.43 \times 10^{0}-2.00 \times 10^{1}$ & $9.746 \times 10^{1}$ & $1.948 \times 10^{5}$ & $2.135 \times 10^{4}$ \\
\hline 2 & $3.00 \times 10^{0}-6.43 \times 10^{0}$ & $1.225 \times 10^{3}$ & $3.850 \times 10^{6}$ & $4.243 \times 10^{5}$ \\
\hline 3 & $1.85 \times 10^{0}-3.00 \times 10^{0}$ & $1.462 \times 10^{3}$ & $7.106 \times 10^{6}$ & $7.856 \times 10^{5}$ \\
\hline 4 & $1.40 \times 10^{0}-1.85 \times 10^{0}$ & $7.496 \times 10^{2}$ & $2.633 \times 10^{6}$ & $2.903 \times 10^{5}$ \\
\hline 5 & $9.00 \times 10^{-1}-1.40 \times 10^{0}$ & $9.662 \times 10^{2}$ & $2.574 \times 10^{6}$ & $2.830 \times 10^{5}$ \\
\hline 6 & $4.00 \times 10^{-1}-9.00 \times 10^{-1}$ & $1.029 \times 10^{3}$ & $2.244 \times 10^{6}$ & $2.461 \times 10^{5}$ \\
\hline 7 & $1.00 \times 10^{-1}-4.00 \times 10^{-1}$ & $2.012 \times 10^{2}$ & $4.311 \times 10^{5}$ & $4.728 \times 10^{4}$ \\
\hline 8 & $1.70 \times 10^{-2}-1.00 \times 10^{-1}$ & 0.0 & 0.0 & 0.0 \\
\hline 9 & $3.00 \times 10^{-3}-1.70 \times 10^{-2}$ & 0.0 & 0.0 & 0.0 \\
\hline 10 & $5.50 \times 10^{-4}-3.00 \times 10^{-3}$ & 0.0 & 0.0 & 0.0 \\
\hline 11 & $1.00 \times 10^{-4}-5.50 \times 10^{-4}$ & 0.0 & 0.0 & 0.0 \\
\hline 12 & $3.00 \times 10^{-5}-1.00 \times 10^{-4}$ & 0.0 & 0.0 & 0.0 \\
\hline 13 & $1.00 \times 10^{-5}-3.00 \times 10^{-5}$ & 0.0 & 0.0 & 0.0 \\
\hline 14 & $3.05 \times 10^{-6}-1.00 \times 10^{-5}$ & 0.0 & 0.0 & 0.0 \\
\hline 15 & $1.77 \times 10^{-6}-3.05 \times 10^{-6}$ & 0.0 & 0.0 & 0.0 \\
\hline 16 & $1.30 \times 10^{-6}-1.77 \times 10^{-6}$ & 0.0 & 0.0 & 0.0 \\
\hline 17 & $1.13 \times 10^{-6}-1.30 \times 10^{-6}$ & 0.0 & 0.0 & 0.0 \\
\hline 18 & $1.00 \times 10^{-6}-1.13 \times 10^{-6}$ & 0.0 & 0.0 & 0.0 \\
\hline 19 & $8.00 \times 10^{-7}-1.00 \times 10^{-6}$ & 0.0 & 0.0 & 0.0 \\
\hline 20 & $4.00 \times 10^{-7}-8.00 \times 10^{-7}$ & 0.0 & 0.0 & 0.0 \\
\hline 21 & $3.25 \times 10^{-7}-4.00 \times 10^{-7}$ & 0.0 & 0.0 & 0.0 \\
\hline 22 & $2.25 \times 10^{-7}-3.25 \times 10^{-7}$ & 0.0 & 0.0 & 0.0 \\
\hline 23 & $1.00 \times 10^{-7}-2.25 \times 10^{-7}$ & 0.0 & 0.0 & 0.0 \\
\hline 24 & $5.00 \times 10^{-8}-1.00 \times 10^{-7}$ & 0.0 & 0.0 & 0.0 \\
\hline 25 & $3.00 \times 10^{-8}-5.00 \times 10^{-8}$ & 0.0 & 0.0 & 0.0 \\
\hline 26 & $1.00 \times 10^{-8}-3.00 \times 10^{-8}$ & 0.0 & 0.0 & 0.0 \\
\hline \multirow[t]{2}{*}{27} & $1.00 \times 10^{-11}-1.00 \times 10^{-8}$ & 0.0 & 0.0 & 0.0 \\
\hline & & $5.730 \times 10^{3}$ & $1.903 \times 10^{7}$ & $2.098 \times 10^{6}$ \\
\hline
\end{tabular}


Table 3.5. Neutron source intensity for fresh fuel

\begin{tabular}{cclc}
\hline & & neutrons/sec & \\
\hline Nuclide & MOX-R & MOX-W & UOX \\
\hline${ }^{234} \mathrm{U}$ & $1.25 \times 10^{1}$ & $1.25 \times 10^{1}$ & $5.02 \times 10^{2}$ \\
${ }^{235} \mathrm{U}$ & $6.66 \times 10^{-1}$ & $6.78 \times 10^{-1}$ & $1.42 \times 10^{1}$ \\
${ }^{238} \mathrm{U}$ & $5.61 \times 10^{3}$ & $5.72 \times 10^{3}$ & $5.22 \times 10^{3}$ \\
${ }^{236} \mathrm{Pu}$ & $1.21 \times 10^{3}$ & $1.21 \times 10^{1}$ & \\
${ }^{238} \mathrm{Pu}$ & $7.07 \times 10^{6}$ & $6.48 \times 10^{4}$ & \\
${ }^{239} \mathrm{Pu}$ & $6.54 \times 10^{5}$ & $7.31 \times 10^{5}$ & \\
${ }^{240} \mathrm{Pu}$ & $7.81 \times 10^{6}$ & $1.28 \times 10^{6}$ & \\
${ }^{241} \mathrm{Pu}$ & $3.66 \times 10^{3}$ & $4.71 \times 10^{1}$ & \\
${ }^{242} \mathrm{Pu}$ & $2.45 \times 10^{6}$ & $9.55 \times 10^{3}$ & \\
${ }^{241} \mathrm{Am}$ & $1.03 \times 10^{6}$ & $1.09 \times 10^{4}$ & \\
Total & $1.90 \times 10^{7}$ & $2.10 \times 10^{6}$ & $5.73 \times 10^{3}$ \\
\hline
\end{tabular}

Task IIb entitled 'Shielding and Heat Generation Study of Fresh Fuel with a Cask' was a study of a cask model typical of those used to transport fissile assemblies of fresh fuel. The cross-section libraries used were the 27-group burnup library (based on ENDF/B-IV) and the 27 neutron, 18-gamma-group library. The fissile assembly (FA) model for fresh fuel is shown in Figure 3.1, and the homogeneous fissile assembly compositions are given in Table 3.9. The model of the cask for fresh fuel is illustrated in Figure 3.2. The atom composition of the structural materials in the cask is given in Table 3.10. Dose rates at the surface of the cask and at $0.5,1$ and 2 meters from the surface were calculated using the SAS2 module of SCALE 4.3R. All three types of fuel were evaluated. The dose rate results are presented in Tables 3.11-3.13. Although the total sources for MOX-W and MOX-R are about an order of magnitude different, the gamma dose rates for MOX-R are higher by approximately a factor of 3 , and the neutron dose rates are higher by approximately a factor of 9. A cursory inspection of the gamma sources in Table 3.6 indicates some groupwise sources differ by up to a factor of 20, while others are nearly the same. These differences are indicative of isotopic differences, but specific scenarios were not analyzed.

In addition to calculating dose rates, a heat generation study for the fresh fuel cask was done using the SAS2 module from SCALE 4.3R with post processing by ORIGEN. The results for the various actinides are presented in Table 3.14. 
Table 3.6. Gamma source spectrum for fresh fuel

\begin{tabular}{|c|c|c|c|c|c|c|}
\hline \multirow[b]{2}{*}{ Energy interval in $\mathrm{MeV}$} & \multicolumn{2}{|c|}{ UOX } & \multicolumn{2}{|c|}{ MOX-R } & \multicolumn{2}{|c|}{ MOX-W } \\
\hline & photons / sec & $\mathrm{mev} / \mathrm{sec}$ & photons / sec & $\mathrm{mev} / \mathrm{sec}$ & photons / sec & $\mathrm{mev} / \mathrm{sec}$ \\
\hline $1.0000 \times 10^{-2}$ to $5.0000 \times 10^{-2}$ & $2.1328 \times 10^{9}$ & $6.3984 \times 10^{7}$ & $2.1379 \times 10^{13}$ & $6.4137 \times 10^{11}$ & $1.3852 \times 10^{12}$ & $4.1555 \times 10^{10}$ \\
\hline $5.0000 \times 10^{-2}$ to $1.0000 \times 10^{-1}$ & $1.5640 \times 10^{8}$ & $1.1730 \times 10^{7}$ & $1.2641 \times 10^{13}$ & $9.4807 \times 10^{11}$ & $1.4063 \times 10^{11}$ & $1.0547 \times 10^{10}$ \\
\hline $1.0000 \times 10^{-1}$ to $2.0000 \times 10^{-1}$ & $1.2326 \times 10^{9}$ & $1.8489 \times 10^{8}$ & $2.3288 \times 10^{10}$ & $3.4932 \times 10^{9}$ & $3.9189 \times 10^{9}$ & $5.8784 \times 10^{8}$ \\
\hline $2.0000 \times 10^{-1}$ to $3.0000 \times 10^{-1}$ & $6.5511 \times 10^{7}$ & $1.6378 \times 10^{7}$ & $6.9238 \times 10^{8}$ & $1.7309 \times 10^{8}$ & $2.8270 \times 10^{8}$ & $7.0674 \times 10^{7}$ \\
\hline $3.0000 \times 10^{-1}$ to $4.0000 \times 10^{-1}$ & $1.9528 \times 10^{6}$ & $6.8348 \times 10^{5}$ & $2.2881 \times 10^{9}$ & $8.0082 \times 10^{8}$ & $1.9354 \times 10^{9}$ & $6.7738 \times 10^{8}$ \\
\hline $4.0000 \times 10^{-1}$ to $6.0000 \times 10^{-1}$ & $1.7470 \times 10^{5}$ & $8.7348 \times 10^{4}$ & $6.3942 \times 10^{8}$ & $3.1971 \times 10^{8}$ & $6.3055 \times 10^{8}$ & $3.1528 \times 10^{8}$ \\
\hline $6.0000 \times 10^{-1}$ to $8.0000 \times 10^{-1}$ & $2.4208 \times 10^{4}$ & $1.6946 \times 10^{4}$ & $5.0810 \times 10^{8}$ & $3.5567 \times 10^{8}$ & $4.2056 \times 10^{7}$ & $2.9439 \times 10^{7}$ \\
\hline $8.0000 \times 10^{-1}$ to $1.0000 \times 10^{0}$ & $3.7240 \times 10^{3}$ & $3.3516 \times 10^{3}$ & $2.0225 \times 10^{7}$ & $1.8202 \times 10^{7}$ & $5.9349 \times 10^{5}$ & $5.3414 \times 10^{5}$ \\
\hline $1.0000 \times 10^{0}$ to $1.3300 \times 10^{0}$ & $5.0684 \times 10^{3}$ & $5.9047 \times 10^{3}$ & $1.2229 \times 10^{7}$ & $1.4247 \times 10^{7}$ & $1.0921 \times 10^{6}$ & $1.2723 \times 10^{6}$ \\
\hline $1.3300 \times 10^{0}$ to $1.6600 \times 10^{0}$ & $2.8316 \times 10^{-22}$ & $4.2333 \times 10^{-22}$ & $1.4498 \times 10^{-22}$ & $2.1675 \times 10^{-22}$ & $1.4533 \times 10^{-22}$ & $2.1727 \times 10^{-22}$ \\
\hline $1.6600 \times 10^{0}$ to $2.0000 \times 10^{0}$ & $2.2097 \times 10^{3}$ & $4.0437 \times 10^{3}$ & $4.2720 \times 10^{6}$ & $7.8177 \times 10^{6}$ & $4.5942 \times 10^{5}$ & $8.4074 \times 10^{5}$ \\
\hline $2.0000 \times 10^{0}$ to $2.5000 \times 10^{0}$ & $1.3366 \times 10^{3}$ & $3.0074 \times 10^{3}$ & $2.5613 \times 10^{6}$ & $5.7629 \times 10^{6}$ & $2.7473 \times 10^{5}$ & $6.1814 \times 10^{5}$ \\
\hline $2.5000 \times 10^{0}$ to $3.0000 \times 10^{0}$ & $7.7344 \times 10^{2}$ & $2.1270 \times 10^{3}$ & $1.4706 \times 10^{6}$ & $4.0442 \times 10^{6}$ & $1.5738 \times 10^{5}$ & $4.3279 \times 10^{5}$ \\
\hline $3.0000 \times 10^{0}$ to $4.0000 \times 10^{0}$ & $6.9336 \times 10^{2}$ & $2.4267 \times 10^{3}$ & $1.3063 \times 10^{6}$ & $4.5721 \times 10^{6}$ & $1.3941 \times 10^{5}$ & $4.8793 \times 10^{5}$ \\
\hline $4.0000 \times 10^{0}$ to $5.0000 \times 10^{0}$ & $2.3348 \times 10^{2}$ & $1.0507 \times 10^{3}$ & $4.3528 \times 10^{5}$ & $1.9588 \times 10^{6}$ & $4.6304 \times 10^{4}$ & $2.0837 \times 10^{5}$ \\
\hline $5.0000 \times 10^{0}$ to $6.5000 \times 10^{0}$ & $9.3505 \times 10^{1}$ & $5.3766 \times 10^{2}$ & $1.7276 \times 10^{5}$ & $9.9338 \times 10^{5}$ & $1.8327 \times 10^{4}$ & $1.0538 \times 10^{5}$ \\
\hline $6.5000 \times 10^{0}$ to $8.0000 \times 10^{0}$ & $1.8308 \times 10^{1}$ & $1.3273 \times 10^{2}$ & $3.3551 \times 10^{4}$ & $2.4325 \times 10^{5}$ & $3.5502 \times 10^{3}$ & $2.5739 \times 10^{4}$ \\
\hline $8.0000 \times 10^{0}$ to $1.0000 \times 10^{1}$ & $3.8821 \times 10^{0}$ & $3.4939 \times 10^{1}$ & $7.0745 \times 10^{3}$ & $6.3670 \times 10^{4}$ & $7.4725 \times 10^{2}$ & $6.7253 \times 10^{3}$ \\
\hline Totals & $3.5895 \times 10^{9}$ & $2.7780 \times 10^{8}$ & $3.4047 \times 10^{13}$ & $1.5946 \times 10^{12}$ & $1.5326 \times 10^{12}$ & $5.3788 \times 10^{10}$ \\
\hline
\end{tabular}


Table 3.7. Total neutron and gamma source from SAS2

\begin{tabular}{lccc}
\hline \multicolumn{4}{c}{ Source spectra for fresh fuel } \\
\hline \multicolumn{1}{c}{ Particle Type } & UOX & MOX-W & MOX-R \\
\hline Neutron $(\mathrm{n} / \mathrm{sec})$ & $5.73 \times 10^{3}$ & $2.10 \times 10^{6}$ & $1.90 \times 10^{7}$ \\
Gammas $(\mathrm{MeV} / \mathrm{sec})$ & $2.78 \times 10^{8}$ & $5.38 \times 10^{10}$ & $1.59 \times 10^{12}$ \\
\hline
\end{tabular}

Table 3.8. Masses of actinides for a fissile assembly of fresh fuel

\begin{tabular}{cccc}
\hline Nuclide & $\begin{array}{c}\text { MOX-R } \\
\text { Grams }\end{array}$ & $\begin{array}{c}\text { MOX-W } \\
\text { Grams }\end{array}$ & $\begin{array}{c}\text { UOX } \\
\text { Grams }\end{array}$ \\
\hline${ }^{16} \mathrm{O}$ & $5.90 \times 10^{4}$ & $5.90 \times 10^{4}$ & $5.38 \times 10^{4}$ \\
${ }^{234} \mathrm{U}$ & $4.08 \times 10^{0}$ & $4.08 \times 10^{0}$ & $1.63 \times 10^{2}$ \\
${ }^{235} \mathrm{U}$ & $8.35 \times 10^{2}$ & $8.51 \times 10^{2}$ & $1.78 \times 10^{4}$ \\
${ }^{238} \mathrm{U}$ & $4.11 \times 10^{5}$ & $4.19 \times 10^{5}$ & $3.82 \times 10^{5}$ \\
${ }^{236} \mathrm{Pu}$ & $2.09 \times 10^{-3}$ & $2.09 \times 10^{-5}$ & \\
${ }^{238} \mathrm{Pu}$ & $4.12 \times 10^{2}$ & $3.78 \times 10^{0}$ & \\
${ }^{239} \mathrm{Pu}$ & $1.58 \times 10^{4}$ & $1.77 \times 10^{4}$ & \\
${ }^{240} \mathrm{Pu}$ & $6.67 \times 10^{3}$ & $1.09 \times 10^{3}$ & \\
${ }^{241} \mathrm{Pu}$ & $2.61 \times 10^{3}$ & $3.36 \times 10^{1}$ & \\
${ }^{242} \mathrm{Pu}$ & $1.43 \times 10^{3}$ & $5.58 \times 10^{0}$ & \\
${ }^{241} \mathrm{Am}$ & $3.51 \times 10^{2}$ & $3.72 \times 10^{0}$ & \\
\hline
\end{tabular}

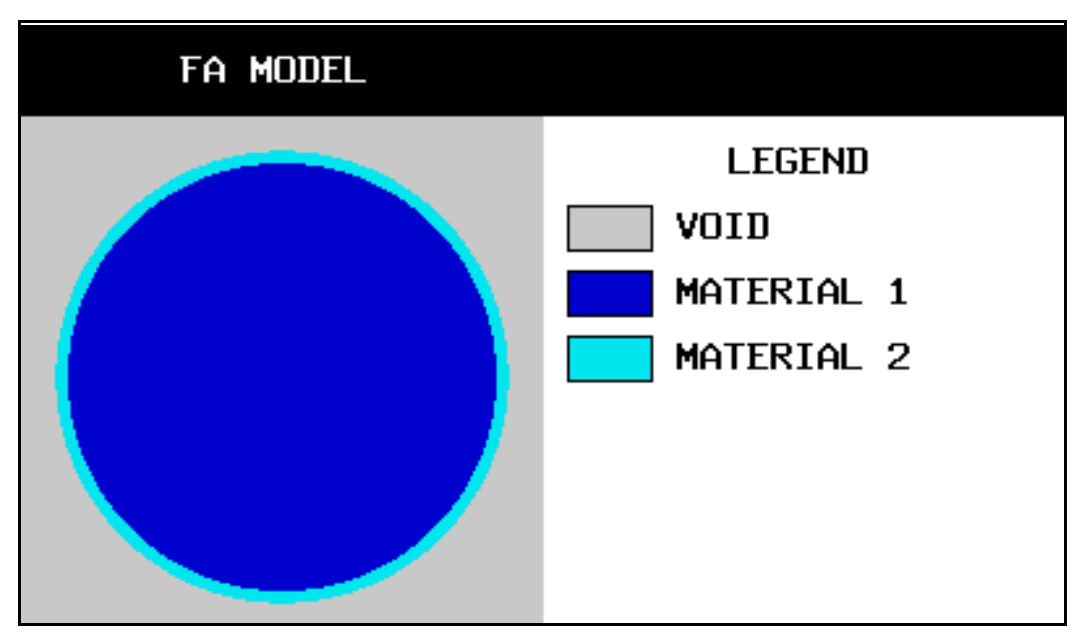

Fig. 3.1. Fissile assembly model. 
Table 3.9. Atom composition of cylinder model of FA in the cask for fresh fuel [atoms/(b cm)]

\begin{tabular}{ccccc}
\hline Zone & & Material 1 & Material 2 \\
\hline Material & MOX-W $(4.2 \%)$ & MOX-R $(6.1 \%)$ & UOX $(4.4 \%)$ & Zr \\
\hline $\mathrm{R}, \mathrm{cm}$ & 12.3 & 12.3 & 12.3 & 12.37 \\
\hline $\mathrm{Zr}$ & 0.004834 & 0.004834 & 0.004834 & 0.04230 \\
${ }^{16} \mathrm{O}$ & $1.3224 \times 10^{-2}$ & $1.3228 \times 10^{-2}$ & $1.2056 \times 10^{-2}$ & \\
${ }^{234} \mathrm{U}$ & $<6.0 \times 10^{-8}$ & $<6.0 \times 10^{-8}$ & $2.5 \times 10^{-6}$ & \\
${ }^{235} \mathrm{U}$ & $1.2832 \times 10^{-5}$ & $1.2587 \times 10^{-5}$ & $2.6846 \times 10^{-4}$ & \\
${ }^{238} \mathrm{U}$ & $6.3224 \times 10^{-3}$ & $6.2016 \times 10^{-3}$ & $5.7595 \times 10^{-3}$ & \\
${ }^{236} \mathrm{Pu}$ & $<3.0 \times 10^{-13}$ & $<3.0 \times 10^{-11}$ & & \\
${ }^{238} \mathrm{Pu}$ & $5.5582 \times 10^{-8}$ & $6.0594 \times 10^{-6}$ & & \\
${ }^{239} \mathrm{Pu}$ & $2.5998 \times 10^{-4}$ & $2.3251 \times 10^{-4}$ & & \\
${ }^{240} \mathrm{Pu}$ & $1.6012 \times 10^{-5}$ & $9.8145 \times 10^{-5}$ & & \\
${ }^{241} \mathrm{Pu}$ & $4.9403 \times 10^{-7}$ & $3.8298 \times 10^{-5}$ & & \\
${ }^{242} \mathrm{Pu}$ & $8.1995 \times 10^{-8}$ & $2.1056 \times 10^{-5}$ & & \\
${ }^{241} \mathrm{Am}$ & $5.4891 \times 10^{-8}$ & $5.1861 \times 10^{-6}$ & & \\
\hline
\end{tabular}

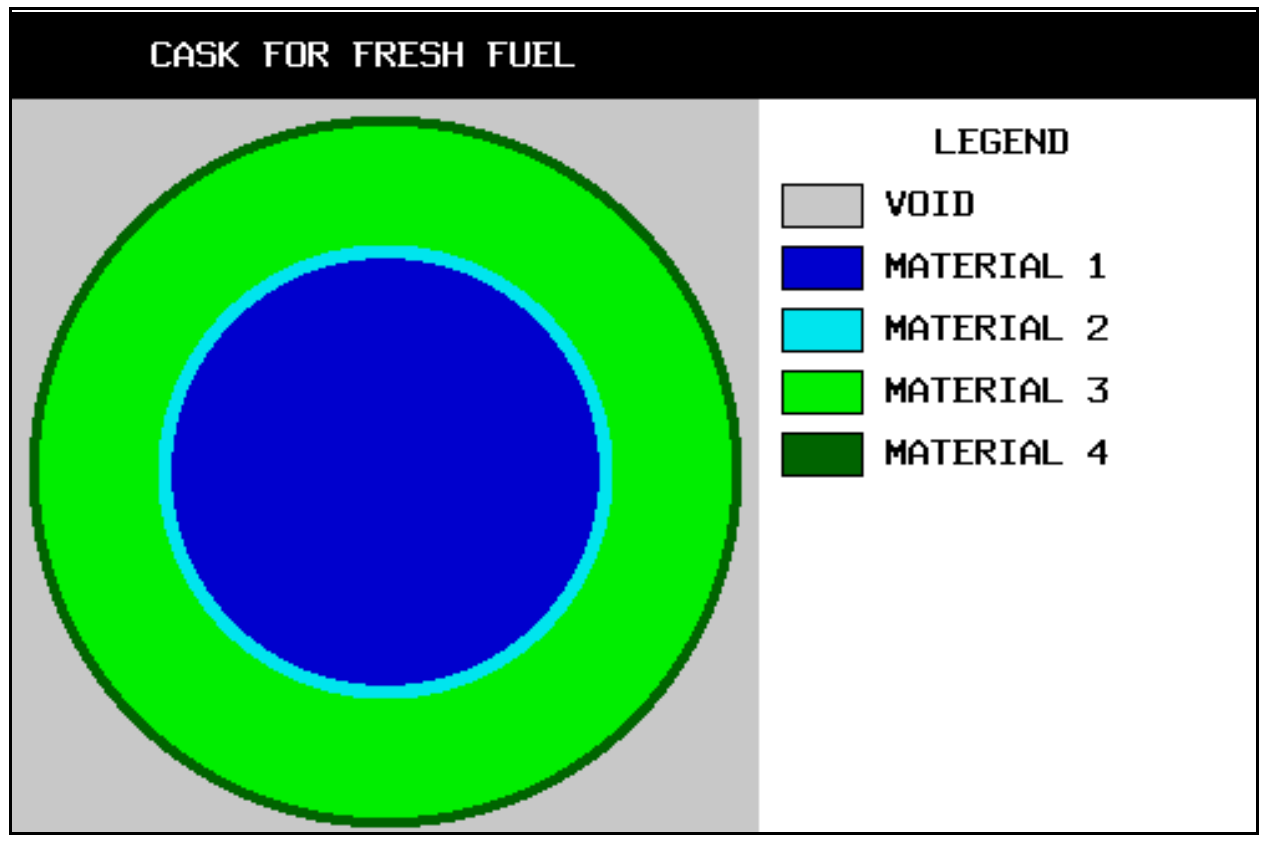

Fig. 3.2. Cask for fresh fuel. 
Table 3.10. Atom composition of structure materials in the cask for fresh fuel [atoms $/(\mathbf{b} \mathbf{~ c m})]$

\begin{tabular}{lcccc}
\hline Zone & Material 1 & Material 2 & Material 3 & Material 4 \\
\hline Material & Air & Stainless steel & Caoutchouc & Stainless steel \\
\hline $\mathrm{R}, \mathrm{cm}$ & 12.6 & 13.4 & 20.4 & 21.0 \\
$\Delta \mathrm{R}, \mathrm{cm}$ & 12.6 & 0.8 & 7.0 & 0.6 \\
$\mathrm{H}$ & & 0.0001 & 0.05372 & \\
$\mathrm{C}$ & & & 0.01791 & 0.0001 \\
$\mathrm{~N}$ & & & \\
$\mathrm{O}$ & & 0.00895 & \\
$\mathrm{Si}$ & & 0.00895 & \\
$\mathrm{Cr}$ & & & 0.01525 \\
$\mathrm{Fe}$ & & & & 0.06006 \\
$\mathrm{Ni}$ & & 0.01525 & & 0.00847 \\
$\mathrm{Ti}$ & & 0.06006 & & 0.00085 \\
\hline
\end{tabular}

Table 3.11. SAS2 results for UOX fresh fuel

\begin{tabular}{lcccc}
\hline \multicolumn{1}{c}{ Detector } & $\begin{array}{c}\text { Flux } \\
\left(\mathrm{n} / \mathrm{cm}^{2} / \mathrm{sec}\right)\end{array}$ & $\begin{array}{c}\text { Dose } \\
(\mathrm{rem} / \mathrm{hr})\end{array}$ & $\begin{array}{c}\text { Flux } \\
\left(\mathrm{photons} / \mathrm{cm}^{2} / \mathrm{sec}\right)\end{array}$ & $\begin{array}{c}\text { Dose } \\
(\mathrm{rem} / \mathrm{hr})\end{array}$ \\
\hline At surface & $1.508 \times 10^{-1}$ & $8.038 \times 10^{-6}$ & $1.723 \times 10^{1}$ & $8.162 \times 10^{-6}$ \\
$0.5 \mathrm{~m}$ from surface & $3.343 \times 10^{-2}$ & $1.889 \times 10^{-6}$ & $4.156 \times 10^{0}$ & $1.985 \times 10^{-6}$ \\
$1 \mathrm{~m}$ from surface & $1.827 \times 10^{-2}$ & $1.044 \times 10^{-6}$ & $2.329 \times 10^{0}$ & $1.112 \times 10^{-6}$ \\
2 m from surface & $8.070 \times 10^{-3}$ & $4.695 \times 10^{-7}$ & $1.081 \times 10^{0}$ & $5.157 \times 10^{-7}$ \\
\hline
\end{tabular}

Table 3.12. SAS2 results for MOX-W fresh fuel

\begin{tabular}{lcccc}
\hline \multicolumn{1}{c}{ Detector } & $\begin{array}{c}\text { Flux } \\
\left(\mathrm{n} / \mathrm{cm}^{2} / \mathrm{sec}\right)\end{array}$ & $\begin{array}{c}\text { Dose } \\
(\mathrm{rem} / \mathrm{hr})\end{array}$ & $\begin{array}{c}\text { Flux } \\
\left(\mathrm{photons} / \mathrm{cm}^{2} / \mathrm{sec}\right)\end{array}$ & $\begin{array}{c}\text { Dose } \\
(\mathrm{rem} / \mathrm{hr})\end{array}$ \\
\hline At surface & $5.467 \times 10^{1}$ & $2.904 \times 10^{-3}$ & $1.265 \times 10^{3}$ & $1.056 \times 10^{-3}$ \\
$0.5 \mathrm{~m}$ from surface & $1.211 \times 10^{1}$ & $6.825 \times 10^{-4}$ & $2.998 \times 10^{2}$ & $2.561 \times 10^{-4}$ \\
$1 \mathrm{~m}$ from surface & $6.623 \times 10^{0}$ & $3.774 \times 10^{-4}$ & $1.664 \times 10^{2}$ & $1.425 \times 10^{-4}$ \\
2 m from surface & $2.928 \times 10^{0}$ & $1.701 \times 10^{-4}$ & $7.522 \times 10^{1}$ & $6.467 \times 10^{-5}$ \\
\hline
\end{tabular}


Table 3.13. SAS2 results for MOX-R fresh fuel

\begin{tabular}{lcccc}
\hline \multicolumn{1}{c}{ Detector } & \multicolumn{2}{c}{ Flux } & \multicolumn{2}{c}{ Gamma } \\
& $\left(\mathrm{n} / \mathrm{cm}^{2} / \mathrm{sec}\right)$ & $\begin{array}{c}\text { Dose } \\
(\mathrm{rem} / \mathrm{hr})\end{array}$ & $\begin{array}{c}\text { Flux } \\
\left(\mathrm{photons} / \mathrm{cm}^{2} / \mathrm{sec}\right)\end{array}$ & $\begin{array}{c}\text { Dose } \\
(\mathrm{rem} / \mathrm{hr})\end{array}$ \\
\hline At surface & $4.945 \times 10^{2}$ & $2.635 \times 10^{-2}$ & $2.845 \times 10^{3}$ & $3.265 \times 10^{-3}$ \\
$0.5 \mathrm{~m}$ from surface & $1.096 \times 10^{2}$ & $6.192 \times 10^{-3}$ & $6.663 \times 10^{2}$ & $7.652 \times 10^{-4}$ \\
1 m from surface & $5.992 \times 10^{1}$ & $3.424 \times 10^{-3}$ & $3.679 \times 10^{2}$ & $4.199 \times 10^{-4}$ \\
2 m from surface & $2.649 \times 10^{1}$ & $1.543 \times 10^{-3}$ & $1.647 \times 10^{2}$ & $1.860 \times 10^{-4}$ \\
\hline
\end{tabular}


Table 3.14. Heat generation for fresh fuel cask

\begin{tabular}{|c|c|c|c|c|c|c|c|c|c|c|c|}
\hline \multirow[b]{3}{*}{ Fuel Type } & \multicolumn{11}{|c|}{ Nuclide thermal power in watts } \\
\hline & \multicolumn{11}{|c|}{ Actinides } \\
\hline & ${ }^{234} \mathrm{U}$ & ${ }^{235} \mathrm{U}$ & ${ }^{237} \mathrm{U}$ & ${ }^{238} \mathrm{U}$ & ${ }^{238} \mathrm{Pu}$ & ${ }^{239} \mathrm{Pu}$ & ${ }^{240} \mathrm{Pu}$ & ${ }^{241} \mathrm{Pu}$ & ${ }^{242} \mathrm{Pu}$ & ${ }^{241} \mathrm{Am}$ & Total \\
\hline UOX & $2.87 \times 10^{-2}$ & $1.05 \times 10^{-3}$ & & $3.25 \times 10^{-3}$ & & & & & & & $3.30 \times 10^{-2}$ \\
\hline MOX-W & & & & $3.57 \times 10^{-3}$ & $2.09 \times 10^{0}$ & $3.34 \times 10^{1}$ & $7.57 \times 10^{0}$ & $1.09 \times 10^{-1}$ & & $4.22 \times 10^{-1}$ & $4.36 \times 10^{1}$ \\
\hline MOX-R & & & $1.26 \times 10^{-7}$ & $3.50 \times 10^{-3}$ & $2.28 \times 10^{2}$ & $2.99 \times 10^{1}$ & $4.64 \times 10^{1}$ & $8.45 \times 10^{0}$ & $1.66 \times 10^{-1}$ & $3.99 \times 10^{1}$ & $3.53 \times 10^{2}$ \\
\hline
\end{tabular}




\section{CALCULATIONAL RESULTS FOR TASK IIc: SHIELDING AND HEAT GENERATION FOR SPENT FUEL WITH A CASK}

Task IIc was a study of a cask model typical of those used to transport fissile assemblies of spent fuel. The cask contained 12 fissile assemblies. A pin irradiation with a burnup of $60 \mathrm{GWd} / \mathrm{MTHM}$ at average linear power of $166 \mathrm{~W} / \mathrm{cm}$ was done. The fuel temperature was $\mathrm{T}=1027 \mathrm{~K}$, and the temperature of the clad and the borated-light-water coolant was $\mathrm{T}=579 \mathrm{~K}$. A cooling time of 3 years was assumed. The densities of the nuclides for the borated water are given in Table 4.1; and the atom composition of the structural materials in the spent fuel cask are presented in Table 4.2. Each of the three types of fuel was analyzed. Dose rates at the surface of the cask and at $0.5,1$ and 2 meters from the surface were calculated using the SAS2 module of SCALE 4.3R. The dose rate results are presented in Tables 4.3-4.5. Initially the gamma dose rates were predicted by the author to be relatively independent of the fuel type. Thus, the higher gamma dose rates for the MOX fuels were surprising. Investigation of these differences revealed that the relatively higher neutron leakage with the MOX fuels as compared with UOX fuel produced more captured gammas; and since nearly $90 \%$ of the gammas are captured gammas, the resulting dose rates for MOX are higher than for LEU.

In addition to calculating dose rates, a heat generation study for the spent fuel cask was done using the ORIGEN module from SCALE 4.3R. The times of disposition used were 3 days, 10 days, and 1,3,10, and 100 years. The results for the various light elements, actinides and fission products are presented in Table 4.6.

The neutron source intensity after 3-year cooling was also calculated and is presented in Table 4.7. Curium 242 is dominant for the (alpha, n), and Curium 244 is dominant for the spontaneous fission. Curium 244 changes slightly for the three types of fuel, but the change is insignificant. Typically (alpha, $\mathrm{n}$ ) results would be about 100 times smaller than the spontaneous fission results. After more years of cooling, the curium 242 would decay out. Table 4.8 shows the total neutron and gamma sources for the spent fuel cask after 3-year cooling.

Table 4.1. Borated-water composition [atoms/(b cm)]

\begin{tabular}{cc}
${ }^{1} \mathrm{H}$ & $4.783 \times 10^{-2}$ \\
${ }^{16} \mathrm{O}$ & $2.391 \times 10^{-2}$ \\
${ }^{10} \mathrm{~B}$ & $4.7344 \times 10^{-6}$ \\
${ }^{11} \mathrm{~B}$ & $1.9177 \times 10^{-5}$ \\
\hline
\end{tabular}


Table 4.2. Atom composition of structure materials in the cask for spent fuel [atoms/(b cm)]

\begin{tabular}{cccc}
\hline Region & 1 & 2 & 3 \\
\hline Diameter, cm & 132 & 200 & 225 \\
$\mathrm{~T}, \mathrm{~K}$ & 523 & 300 & 300 \\
$\mathrm{Zr}$ & 0.002216 & & \\
$\mathrm{Fe}$ & 0.0027 & 0.061 & \\
$\mathrm{Cr}$ & 0.0007 & 0.016 & \\
$\mathrm{Ni}$ & 0.0004 & 0.008 & \\
$\mathrm{~B}$ & 0.00029 & & \\
$\mathrm{O}$ & 0.0054 & & 0.026 \\
$\mathrm{C}$ & & & 0.014 \\
$\mathrm{H}$ & & & 0.065 \\
\hline
\end{tabular}

Table 4.3. SAS2 results for UOX spent fuel

\begin{tabular}{lcccc}
\hline \multicolumn{1}{c}{ Detector } & \multicolumn{2}{c}{ Neutron } & \multicolumn{2}{c}{ Gamma } \\
& $\left(\mathrm{n} / \mathrm{cm}^{2} / \mathrm{sec}\right)$ & $\begin{array}{c}\text { Dose } \\
(\mathrm{rem} / \mathrm{hr})\end{array}$ & $\begin{array}{c}\text { Flux } \\
\left(\mathrm{photons} / \mathrm{cm}^{2} / \mathrm{sec}\right)\end{array}$ & $\begin{array}{c}\text { Dose } \\
(\mathrm{rem} / \mathrm{hr})\end{array}$ \\
\hline At surface & $8.227 \times 10^{2}$ & $5.628 \times 10^{-3}$ & $1.837 \times 10^{4}$ & $3.345 \times 10^{-2}$ \\
$0.5 \mathrm{~m}$ from surface & $4.254 \times 10^{2}$ & $3.082 \times 10^{-3}$ & $9.945 \times 10^{3}$ & $1.881 \times 10^{-2}$ \\
$1 \mathrm{~m}$ from surface & $2.881 \times 10^{2}$ & $2.142 \times 10^{-3}$ & $6.789 \times 10^{3}$ & $1.289 \times 10^{-2}$ \\
$2 \mathrm{~m}$ from surface & $1.508 \times 10^{2}$ & $1.276 \times 10^{-3}$ & $3.634 \times 10^{3}$ & $6.941 \times 10^{-3}$ \\
\hline
\end{tabular}

Table 4.4. SAS2 results for MOX-W spent fuel

\begin{tabular}{|c|c|c|c|c|}
\hline \multirow[b]{2}{*}{ Detector } & \multicolumn{2}{|c|}{ Neutron } & \multicolumn{2}{|c|}{ Gamma } \\
\hline & $\begin{array}{c}\text { Flux } \\
\left(\mathrm{n} / \mathrm{cm}^{2} / \mathrm{sec}\right)\end{array}$ & $\begin{array}{c}\text { Dose } \\
(\mathrm{rem} / \mathrm{hr})\end{array}$ & $\begin{array}{c}\text { Flux } \\
\text { (photons } / \mathrm{cm}^{2} / \mathrm{sec} \text { ) }\end{array}$ & $\begin{array}{c}\text { Dose } \\
(\mathrm{rem} / \mathrm{hr})\end{array}$ \\
\hline At surface & $2.189 \times 10^{3}$ & $1.497 \times 10^{-2}$ & $3.245 \times 10^{4}$ & $6.903 \times 10^{-2}$ \\
\hline $0.5 \mathrm{~m}$ from surface & $1.132 \times 10^{3}$ & $8.197 \times 10^{-3}$ & $1.718 \times 10^{4}$ & $3.775 \times 10^{-2}$ \\
\hline $1 \mathrm{~m}$ from surface & $7.665 \times 10^{2}$ & $5.696 \times 10^{-3}$ & $1.155 \times 10^{4}$ & $2.536 \times 10^{-2}$ \\
\hline $2 \mathrm{~m}$ from surface & $4.012 \times 10^{2}$ & $3.126 \times 10^{-3}$ & $6.008 \times 10^{3}$ & $1.312 \times 10^{-2}$ \\
\hline
\end{tabular}


Table 4.5. SAS2 results for MOX-R spent fuel

\begin{tabular}{lcccc}
\hline \multicolumn{1}{c}{ Detector } & $\begin{array}{c}\text { Flux } \\
\left(\mathrm{n} / \mathrm{cm}^{2} / \mathrm{sec}\right)\end{array}$ & $\begin{array}{c}\text { Dose } \\
(\mathrm{rem} / \mathrm{hr})\end{array}$ & $\begin{array}{c}\text { Flux } \\
\left(\mathrm{photons} / \mathrm{cm}^{2} / \mathrm{sec}\right)\end{array}$ & $\begin{array}{c}\text { Dose } \\
(\mathrm{rem} / \mathrm{hr})\end{array}$ \\
\hline At surface & $8.248 \times 10^{3}$ & $5.653 \times 10^{-2}$ & $9.258 \times 10^{4}$ & $2.241 \times 10^{-1}$ \\
$0.5 \mathrm{~m}$ from surface & $4.265 \times 10^{3}$ & $3.096 \times 10^{-2}$ & $4.799 \times 10^{4}$ & $1.202 \times 10^{-1}$ \\
$1 \mathrm{~m}$ from surface & $2.888 \times 10^{3}$ & $2.152 \times 10^{-2}$ & $3.177 \times 10^{4}$ & $7.949 \times 10^{-2}$ \\
2 m from surface & $1.512 \times 10^{3}$ & $1.181 \times 10^{-2}$ & $1.604 \times 10^{4}$ & $3.983 \times 10^{-2}$ \\
\hline
\end{tabular}


Table 4.6. Heat generation for spent fuel cask

\begin{tabular}{|c|c|c|c|c|c|c|c|c|c|c|c|}
\hline \multicolumn{12}{|c|}{ Nuclide thermal power in watts } \\
\hline & \multicolumn{11}{|c|}{ Time of disposition } \\
\hline & Initial & $3.0 \mathrm{~d}$ & $10.0 \mathrm{~d}$ & $30.0 \mathrm{~d}$ & $100.00 \mathrm{~d}$ & $365.0 \mathrm{~d}$ & $1095.0 \mathrm{~d}$ & $3650.0 \mathrm{~d}$ & $9000.0 \mathrm{~d}$ & $27000.0 \mathrm{~d}$ & $36500.0 \mathrm{~d}$ \\
\hline \multicolumn{12}{|c|}{ Light Elements } \\
\hline UOX & $1.05 \times 10^{3}$ & $3.42 \times 10^{2}$ & $2.94 \times 10^{2}$ & $2.58 \times 10^{2}$ & $1.62 \times 10^{2}$ & $4.92 \times 10^{1}$ & $3.07 \times 10^{1}$ & $1.19 \times 10^{1}$ & $1.72 \times 10^{0}$ & $5.60 \times 10^{-3}$ & $2.60 \times 10^{-3}$ \\
\hline MOX-R & $9.10 \times 10^{2}$ & $2.89 \times 10^{2}$ & $2.44 \times 10^{2}$ & $2.13 \times 10^{2}$ & $1.32 \times 10^{2}$ & $3.73 \times 10^{1}$ & $2.25 \times 10^{1}$ & $8.65 \times 10^{0}$ & $1.24 \times 10^{0}$ & $3.72 \times 10^{-3}$ & $1.60 \times 10^{-3}$ \\
\hline MOX-W & $9.87 \times 10^{2}$ & $3.15 \times 10^{2}$ & $2.67 \times 10^{2}$ & $2.34 \times 10^{2}$ & $1.45 \times 10^{2}$ & $4.15 \times 10^{1}$ & $2.52 \times 10^{1}$ & $9.72 \times 10^{0}$ & $1.40 \times 10^{0}$ & $4.29 \times 10^{-3}$ & $1.89 \times 10^{-3}$ \\
\hline \multicolumn{12}{|l|}{ Actinides } \\
\hline UOX & $6.69 \times 10^{4}$ & $1.54 \times 10^{4}$ & $3.75 \times 10^{3}$ & $1.67 \times 10^{3}$ & $1.27 \times 10^{3}$ & $6.21 \times 10^{2}$ & $3.16 \times 10^{2}$ & $2.79 \times 10^{2}$ & $2.41 \times 10^{2}$ & $1.69 \times 10^{2}$ & $1.49 \times 10^{2}$ \\
\hline MOX-R & $7.19 \times 10^{4}$ & $2.28 \times 10^{4}$ & $1.32 \times 10^{4}$ & $1.10 \times 10^{4}$ & $8.69 \times 10^{3}$ & $4.22 \times 10^{3}$ & $2.10 \times 10^{3}$ & $1.72 \times 10^{3}$ & $1.31 \times 10^{3}$ & $7.29 \times 10^{2}$ & $6.21 \times 10^{2}$ \\
\hline MOX-W & $6.89 \times 10^{4}$ & $1.78 \times 10^{4}$ & $7.12 \times 10^{3}$ & $5.14 \times 10^{3}$ & $3.97 \times 10^{3}$ & $1.72 \times 10^{3}$ & $6.72 \times 10^{2}$ & $5.74 \times 10^{2}$ & $4.91 \times 10^{2}$ & $3.51 \times 10^{2}$ & $3.17 \times 10^{2}$ \\
\hline \multicolumn{12}{|c|}{ Fission Products } \\
\hline UOX & $7.46 \times 10^{5}$ & $6.06 \times 10^{4}$ & $4.18 \times 10^{4}$ & $2.65 \times 10^{4}$ & $1.46 \times 10^{4}$ & $6.25 \times 10^{3}$ & $2.19 \times 10^{3}$ & $6.77 \times 10^{2}$ & $4.16 \times 10^{2}$ & $1.25 \times 10^{2}$ & $6.70 \times 10^{1}$ \\
\hline MOX-R & $7.23 \times 10^{5}$ & $5.95 \times 10^{4}$ & $4.14 \times 10^{4}$ & $2.66 \times 10^{4}$ & $1.52 \times 10^{4}$ & $7.03 \times 10^{3}$ & $2.36 \times 10^{3}$ & $6.01 \times 10^{2}$ & $3.54 \times 10^{2}$ & $1.05 \times 10^{2}$ & $5.67 \times 10^{1}$ \\
\hline MOX-W & $7.31 \times 10^{5}$ & $6.07 \times 10^{4}$ & $4.22 \times 10^{4}$ & $2.71 \times 10^{4}$ & $1.54 \times 10^{4}$ & $7.12 \times 10^{3}$ & $2.41 \times 10^{3}$ & $6.16 \times 10^{2}$ & $3.61 \times 10^{2}$ & $1.07 \times 10^{2}$ & $5.79 \times 10^{1}$ \\
\hline
\end{tabular}


Table 4.7. Neutron source intensity after 3-year cooling

\begin{tabular}{lccc}
\hline & UOX \% & MOX-R \% & MOX-W \% \\
\hline Fraction of $(\alpha, \mathrm{n})$ & & & \\
$\quad{ }^{238} \mathrm{Pu}$ & 4.5 & 2.1 & 1.1 \\
${ }^{242} \mathrm{Cm}$ & 84.9 & 84.6 & 90.4 \\
${ }^{244} \mathrm{Cm}$ & 9.8 & 12.8 & 7.9 \\
Fraction of SF & & & \\
${ }^{238} \mathrm{Pu}$ & 0.05 & 0.01 & 0.01 \\
${ }^{242} \mathrm{Cm}$ & 24.4 & 18.6 & 29.5 \\
${ }^{244} \mathrm{Cm}$ & 73.4 & 73.6 & 67.5 \\
${ }^{246} \mathrm{Cm}$ & 0.7 & 0.1 & 0.7 \\
${ }^{252} \mathrm{Cf}$ & 1.4 & 6.6 & 2.1 \\
Fraction of total & & & \\
${ }^{242} \mathrm{Cm}$ & 27.6 & 21.3 & 33.3 \\
${ }^{244} \mathrm{Cm}$ & 69.8 & 71.2 & 63.8 \\
\hline
\end{tabular}

Table 4.8. Total neutron and gamma source from SAS2

\begin{tabular}{lccc}
\hline \multicolumn{4}{c}{ Source for spent fuel after 3-year cooling } \\
\hline \multicolumn{1}{c}{ Particle Type } & UOX & MOX-W & MOX-R \\
\hline Neutron $(\mathrm{n} / \mathrm{sec})$ & $6.83 \times 10^{8}$ & $1.81 \times 10^{9}$ & $6.37 \times 10^{9}$ \\
Gammas $(\mathrm{MeV} / \mathrm{sec})$ & $6.37 \times 10^{15}$ & $7.29 \times 10^{15}$ & $6.99 \times 10^{15}$ \\
\hline
\end{tabular}




\section{CONCLUSIONS}

The ORNL results for these benchmarks differ in some ways from the Russian results which are given in Appendices B and C. The Russians used several different computer codes to do each of the calculations. When comparing calculations, the more similarities in the methods used, the better the comparison should be. Therefore, since all of the ORNL calculations were done using ENDF/B-V cross sections, whenever possible comparisons were made to Russian results that also used $\mathrm{ENDF} / \mathrm{B}-\mathrm{V}$ cross sections.

For Task I, both UOX and MOX-weapons grade results were compared to the Russian IPPE MCNP-BV/C calculations that used ENDF/B-V cross section data for the k-effective calculations. The MOX-reactor grade results were compared to the MCU/MCUDAT results because there were no reported Russian results using ENDF/B-V data. Analysis of the differences indicate that the $k_{\text {eff }}$ for all three types of fuel differ by less than $1.2 \%$ for water densities down to $0.3 \mathrm{~g} / \mathrm{cc}$. Densities below this produce increasingly larger percentage differences, with the dry case, i.e., with void (no water anywhere) in the geometry, differing by $30-40 \%$. The ORNL result is significantly lower for the dry cases; however, when ORNL ran cases eliminating the void space around the assemblies, the results were within $1 \%$ of the Russian results. Appendix E has the results of the KENO-VI calculation which does not contain a void region between assemblies. For MOX fuel, it is noteworthy that the local maximum multiplication factor for low water densities occurs at a higher $\mathrm{H} /$ fissile atom ratio in ORNL calculations than in Russian calculations. While the difference is slight, the result is noteworthy for dry storage safety analyses.

Comparison of the neutron source for Task IIa reveals that the ORNL result differs from the Russian results by less than $1 \%$ for UOX and MOX-R and by approximately $3 \%$ for MOX-W. The gamma source in photons/sec cannot be directly compared to the Russian result because of the differences in group structures; in particular, because the mean energy of the lowest energy group is quite different, the source in this group is skewed. In order to try to understand the differences, ORNL ran an ORIGEN-ARP case which has a gamma group structure more like the Russian structure; results agreed to within 5-6\%. Comparison of the neutron source intensity indicates that for UOX the ORNL results for the individual isotopes differ by no more than $0.5 \%$ from the Russian IPPE-Z results, and the total intensity differs by no more than $1 \%$ from all three Russian calculations. For the mixed oxide fuels, all the isotopes that contribute a significant amount differ by no more than $1 \%$ from the IPPE-Z results. The ORNL ORIGEN-ARP results are all within $2 \%$ of the SAS2 results.

A comparison of the neutron dose rate result at the surface for UOX from the Task IIa calculations reveals that the ORNL neutron dose result is within $1 \%$ of the Russian IPPE-K (CARE+ANISN) result. Preliminary analysis of the gamma results at the surface of the UOX indicate a difference of about a factor of 9 but that applying an estimated equilibrium factor (in other words, including in the calculation daughter products at equilibrium concentrations which occur in naturally occurring uranium) to the ORNL results brings them within a factor of 1.5 of the Russian results. The neutron and gamma dose rate results for UOX at other detector locations cannot be individually compared because the reported Russian results are for total dose only. The total dose varies significantly because of the gamma results; however, as the distance to detector increases, the percentage of difference decreases. 
For Task IIb calculations, neutron dose rates at the surface differ from the Russian results by 5\% to 9\%; and the ORNL gamma dose rates for the MOX-weapon grade falls between the Russian IPPE-K and the IPPE-Z results while the MOX-reactor grade is about $17 \%$ less than the IPPE-Z results. The UOX gamma dose rate is different by approximately a factor of 30 . Because the Russian and Oak Ridge gamma crosssection libraries that were used do not have the same group structure, it is difficult to find all the reasons for the differences. Part of the difference for the UOX is caused by the fact that ORNL made no equilibrium assumptions for the daughter products of ${ }^{238} \mathrm{U}$ while the Russians took this into account. ORNL did not apply this because it was not specified in the problem description. Since most of the gamma source comes from ${ }^{238} \mathrm{U}$ due to the fact that each ${ }^{238} \mathrm{U}$ decay produces two gammas (nuclides ${ }^{234} \mathrm{Th}$ and ${ }^{234 \mathrm{~m}} \mathrm{~Pa}$ ), there is a significant effect. As in the case for Task IIa, the neutron and gamma dose rates at the other detectors are not reported by the Russians; only the total dose rate is shown. The differences between the total dose rates calculated by ORNL and the Russians are significant due to the reasons stated above. The ORNL heat generation results are all within $2 \%$ for UOX, $1 \%$ for MOX-R and identical for MOX-W to the Russian results. The masses of the actinides differ by less than $0.5 \%$. The source spectra per energy group is comparable for neutrons, but the gamma results are difficult to compare due to the group structure differences. Additional calculations using the same gamma group structures would likely resolve this problem.

For Task IIc (spent fuel) the neutron source for UOX after 3-year cooling is within $1 \%$ and for MOX-W within 5\% of the Russian IPPE-K result while the MOX-R difference is the largest at over $10 \%$.

Task IIc neutron dose rate for UOX at the surface is within 5\% and for MOX-R within $1 \%$ of that calculated by the Russian IPPE-K method while the MOX-W difference is the largest at over 15\%. The gamma dose rates for UOX differ by about $18 \%$, the MOX-R by about $6 \%$, and the MOX-W by approximately $20 \%$. The MOX-R total dose gives the best comparison for all detectors. Resolution of the differences would require additional calculations using the same gamma group structure by both the Russian and ORNL scientists. The heat generation results vary from being the same to being up to 6\% different from the Russian IPPE-K results which were calculated using the CARE, ANISN and CONSYST computer codes.

The input data files used to generate the results of these benchmarks have been archived and will be made available for additional studies. 


\section{REFERENCES}

1. SCALE: A Modular Code System for Performing Standardized Computer Analyses for Licencing Evaluation, NUREG/CR-0200, Rev. 5 (ORNL/NUREG/CSD-2R5), Vols. I, II, and III, March 1997. Available from Radiation Safety Information Computational Center at Oak Ridge National Laboratory as CCC-545.

2. S. M. Bowman and L. C. Leal, "ORIGEN-ARP: Automatic Rapid Process for Spent Fuel Depletion, Decay, and Source Term Analysis," Vol. I, Sect. D1 of SCALE: A Modular Code System for Performing Standardized Computer Analyses for Licencing Evaluation, NUREG/CR0200, Rev. 6 (ORNL/NUREG/CSD-2R6), Vols. I, II, and III, in 2000. Available later from Radiation Safety Information Computational Center at Oak Ridge National Laboratory as CCC545. 



\section{APPENDIX A \\ DESCRIPTION OF SAFETY ANALYSIS COMPUTATIONAL BENCHMARK}





\title{
APPENDIX A
}

\section{DESCRIPTION OF SAFETY ANALYSIS COMPUTATIONAL BENCHMARK}

\author{
A. Pavlovitchev (RRC KI), \\ A. Kalashnikov, G. Manturov (SSC RF IPPE)
}

\section{A.1 Introduction}

These benchmark specifications were designed to provide a few simple calculational benchmark problems for a MOX lattice that is typical of that which might be used for fissile materials disposition at NPPs with VVER-1000 type reactor.

The proposed calculational benchmark is concerned to criticality safety studies in fresh and spent fuel storage at the reactor and under a transportation of fissile assembly (FA) at NPP.

Fresh fuel storage will require criticality data for the water moderated MOX lattices. The same data should be applicable to certifying physics parameters of MOX fuel in the reactor core. Spent fuel storage should be licensed based on the same data as fresh fuel storage as well and quite the same data might be applicable to the transportation analysis of the FA in a cask.

The benchmark specifications describe two benchmark problems respectively named as Task I and Task II:

- Task I is a criticality safety study in fresh fuel storage,

- Task II is a shielding and heat generation study with a cask.

The benchmark study is concerned to the following type of fuel: MOX weapons-grade and civil plutonium fuel, and a traditional UOX uranium low-enriched fuel.

The FA model chosen was that of a VVER-1000 in assembly lattice of 312 pins all of which have the same initial composition. The assembly also contains 18 control rod guide tubes and a central instrumentation channel.

The storage model is typical of that which might be used at NPP with VVER-1000 type reactor. It is a 1-D infinite FA lattice with a pitch $40 \mathrm{~cm}$.

The cask models for fresh and spent fuel are based on typical of those which might be used for a transportation of FAs. They are different for fresh and spent fuel.

\section{A.2 Task I: Criticality Safety Study in Storage}

In this task a study of criticality safety in fresh fuel storage is performed. It is assumed an infinite pool storage of FAs filled with a cold water. The triangular pitch is $40 \mathrm{~cm}$. The geometric data for FA is given in Table A.1 and in Fig. A.1. The composition of fresh fuel is given in Table A.2. The temperature of FA for fresh condition is $\mathrm{T}=300 \mathrm{~K}$. The cold water composition at $\mathrm{T}=300 \mathrm{~K}$ and $\gamma\left(\mathrm{H}_{2} \mathrm{O}\right)=1 \mathrm{~g} / \mathrm{cm}^{3}$ is assumed [atoms $\left./(\mathrm{b} \mathrm{cm})\right]$ :

$$
\begin{array}{ll}
\mathrm{H} & 0.06694 \\
\mathrm{O} & 0.03347
\end{array}
$$


Several calculations are to be performed for fresh FA for each type of fuel to provide a dependence of criticality parameter $\mathrm{k}_{\text {eff }}$ via water density for $\gamma\left(\mathrm{H}_{2} \mathrm{O}\right)=1.0,0.9,0.8,0.7,0.6$, $0.5,0.4,0.3,0.2,0.1,0.05,0.03,0.02$ and $0[\mathrm{~g} / \mathrm{cm} 3]$ :

- function $k_{\text {eff }}(\gamma)$.

\section{A.3 Task II: Shielding and Heat Generation Study with a Cask} performed.

In this task a study of shielding and radioactive characteristics at a transportation of FA is

The task is divided on three parts:

- Task IIa is a study of radioactive characteristics of fresh fuel,

- Task IIb is shielding and heat generation study of fresh fuel with a cask,

- Task IIc is shielding and heat generation study of spent fuel with a cask.

\section{A.3.1 Task IIa: Study of radioactive characteristics of fresh fuel}

It is assumed a dry assembly of fresh fuel with geometric specifications given in Table A.1 and in Fig. A.1. The composition of fresh fuel is given in Table A.2. The temperature of FA for fresh condition is $\mathrm{T}=300 \mathrm{~K}$. No water is present.

Several calculations are to be performed for each type of fuel to provide shielding and radioactive characteristics:

- Neutron source strength: fractional by separate isotopes and spectrum in used group structure.

- Gamma strength: total and spectrum in used group structure.

- Dose rates at distance from the surface of FA equal to 0, 0.5, 1 and 2 meter.

\section{A.3.2 Task IIb: Shielding and heat generation study of fresh fuel with a cask}

It is assumed a model of a cask for fresh fuel. The cask model is simplified of that which is typical and might be used for a transportation of fresh FA.

Several calculations are to be performed for each type of fuel to provide shielding and radioactive characteristics:

- Dose rates at distance from the surface of the cask equal to 0, 0.5, 1 and 2 meter.

- Heat generation - total and fractional by actinides.

\section{A.3.3 Task IIc: Shielding and heat generation study of spent fuel with a cask}

It is assumed a model of a cask for spent fuel. The cask model is simplified of that which is typical and might be used for a transportation of spent FA. It contains 12 FA.

Before moving FA to the cask it is assumed a storaging of FA in a pool storage like that which was described in Task I. For calculation a spent fuel composition a pin-cell irradiation is to be performed with a discharge burnup of $60 \mathrm{GWd} / \mathrm{MTHM}$ at an average power $166 \mathrm{~W} / \mathrm{cm}$. The pincell cylinder specifications are: $r_{1 \text { fuel }}=0.386 \mathrm{~cm}, r_{2, \text { clad }}=0.4582 \mathrm{~cm}, r_{3, \bmod }=0.7015 \mathrm{~cm}$. The initial fuel compositions are given in Table A.2. For the pin-cell burnup calculations the real composition of light water with boron is given in Table A.3. The operated temperatures should be used: the fuel 
temperature is $\mathrm{T}_{\text {fuel }}=1027 \mathrm{~K}$, the clad temperature is $\mathrm{T}_{\text {clad }}=579 \mathrm{~K}$ and borate-light-water coolant temperature is $\mathrm{T}_{\text {mod }}=579 \mathrm{~K}$.

Several calculations are to be performed for each type of fuel to provide shielding and radioactive characteristics:

- Nuclide composition of actinides and fission products in spent fuel, its activity.

- Neutron source strength: fractional by separate isotopes and spectrum in used group structure.

- Gamma source strength: total and spectrum in used group structure.

- Dose rates at distance from the surface of the cask equal to 0, 0.5, 1 and 2 meter.

- Heat generation - total and fractionally for actinides and fission products in spent fuel (one FA) via time of disposition which is equal to 3 and 10 days, and 1, 3, 10, 100 years.

\section{A.4 Benchmark Specifications}

\section{A.4.1 FA Geometry Data}

The FA geometry is typical of a VVER-1000 assembly. It is a hexagonal FA shown in Fig. A.1. The geometric specifications are presented in Table A.1.

Table A.1. General assembly data

\begin{tabular}{ll}
\hline Parameter & \multicolumn{1}{c}{ Value } \\
\hline Number of fuel pins & 312 \\
Number of guide tubes & 18 \\
Number of instrumentation tubes & 1 \\
Pin pitch, cm & 1.275 \\
Fuel rods & \\
$\quad$ Pellet diameter, cm & 0.772 \\
Clad inside diameter, cm & 0.772 \\
Clad outside diameter, cm & 0.91 \\
Clad material & $\mathrm{Zr}$ \\
$\quad$ Active fuel length, cm & 353 \\
Guide tubes & \\
Inside diameter, cm & 1.09 \\
Outside diameter, cm & 1.265 \\
Material & $\mathrm{Zr}$ \\
Central instrumentation tube & \\
Inside diameter, cm & 0.960 \\
Outside diameter, cm & 1.125 \\
Material & $\mathrm{Zr}$ \\
\hline
\end{tabular}




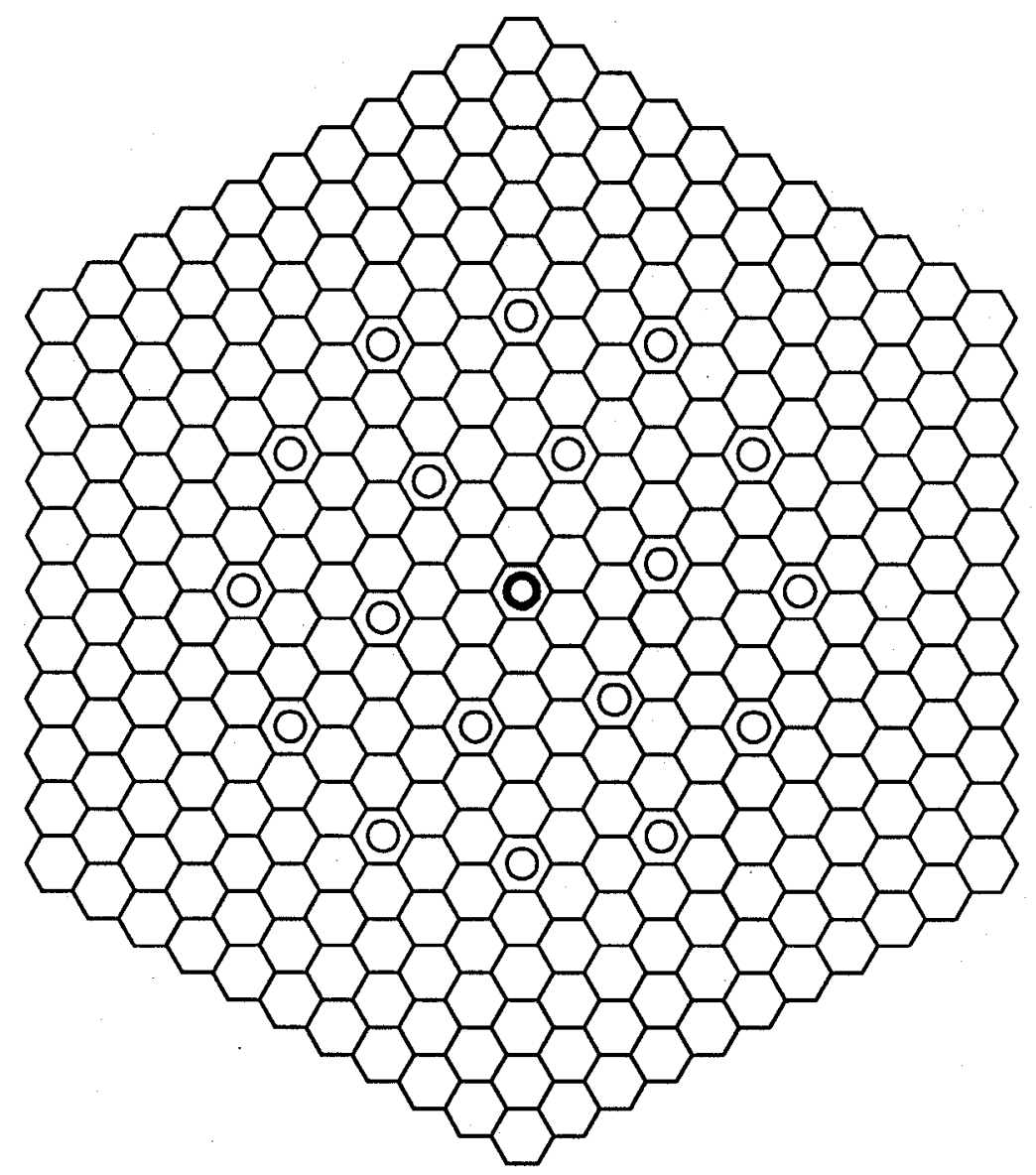

Fuel rod $\quad-312$
(O) Guide tube -18
(O) Central instrumentation tube -1

Fig. A.1. VVER-1000 FA geometry. 


\section{A.4.2 FA Material Data}

Fuel.

Table A. 2 contains fresh fuel compositions for MOX-W (4.2\%) weapons-grade and MOX-R (6.1\%) civil, reactor-grade plutonium, and UOX (4.4\%).

Table A.2. MOX and UOX fresh fuel compositions [atoms/(b cm)]

\begin{tabular}{|c|c|c|c|}
\hline Nuclides & MOX-W (4.2\%) & MOX-R (6.1\%) & UOX (4.4\%) \\
\hline${ }^{16} \mathrm{O}$ & $4.3036 \mathrm{E}-02$ & $4.3051 \mathrm{E}-02$ & $3.9235 \mathrm{E}-02$ \\
\hline${ }^{234} \mathrm{U}$ & $<2.0 \mathrm{E}-07$ & $<2.0 \mathrm{E}-07$ & $8.0 \mathrm{E}-06$ \\
\hline${ }^{235} \mathrm{U}$ & $4.1762 \mathrm{E}-05$ & $4.0964 \mathrm{E}-05$ & $8.7370 \mathrm{E}-04$ \\
\hline${ }^{238} \mathrm{U}$ & $2.0576 \mathrm{E}-02$ & $2.0183 \mathrm{E}-02$ & $1.8744 \mathrm{E}-02$ \\
\hline${ }^{236} \mathrm{Pu}$ & $<1.0 \mathrm{E}-12$ & $<1.0 \mathrm{E}-10$ & \\
\hline${ }^{238} \mathrm{Pu}$ & $1.8089 \mathrm{E}-07$ & $1.9720 \mathrm{E}-05$ & \\
\hline${ }^{239} \mathrm{Pu}$ & $8.4610 \mathrm{E}-04$ & $7.5671 \mathrm{E}-04$ & \\
\hline${ }^{240} \mathrm{Pu}$ & $5.2111 \mathrm{E}-05$ & $3.1941 \mathrm{E}-04$ & \\
\hline${ }^{241} \mathrm{Pu}$ & $1.6078 \mathrm{E}-06$ & $1.2464 \mathrm{E}-04$ & \\
\hline${ }^{242} \mathrm{Pu}$ & $2.6685 \mathrm{E}-07$ & $6.8527 \mathrm{E}-05$ & \\
\hline${ }^{241} \mathrm{Am}$ & $1.7864 \mathrm{E}-07$ & $1.6878 \mathrm{E}-05$ & \\
\hline
\end{tabular}

\section{Cladding.}

For simplicity, a uniform Zirconium composition 0.0423 atoms $/(\mathrm{b} \mathrm{cm})$ is assumed.

\section{Coolant/moderator.}

Light-water coolant density with boron is given in Table A.3.

Table A.3. Borate-water composition [atoms/(b cm)]

\begin{tabular}{|r|r|}
\hline${ }^{1} \mathrm{H}$ & $4.783 \mathrm{E}-02$ \\
\hline${ }^{16} \mathrm{O}$ & $2.391 \mathrm{E}-02$ \\
\hline${ }^{10} \mathrm{~B}$ & $4.7344 \mathrm{E}-06$ \\
\hline${ }^{11} \mathrm{~B}$ & $1.9177 \mathrm{E}-05$ \\
\hline
\end{tabular}




\section{A.4.3 Cask Model for Fresh Fuel}

The cask model for fresh fuel is based on that which is typical for a transportation of 1 fresh FA. The FA cylinder model is shown in Fig. A.2. The homogeneous FA compositions are presented in Table A.4. The geometric specifications and structure materials compositions are presented in Table A.5 and in Fig. A.3. The FA is placed co-centered into the cask.

Table A.4. Atom composition of cylinder model of FA in the cask for fresh fuel [atoms/(b cm)]

\begin{tabular}{|c|c|c|c|c|c|}
\hline & Zone & \multicolumn{3}{|c|}{ Region 1} & Region 2 \\
\hline & Material & MOX-W (4.2\%) & $\begin{array}{c}\text { MOX-R } \\
(6.1 \%)\end{array}$ & UOX (4.4\%) & $\mathrm{Zr}$ \\
\hline & $\mathrm{R}, \mathrm{cm}$ & 12.3 & 12.3 & 12.3 & 12.37 \\
\hline & $\mathrm{Zr}$ & 0.004834 & 0.004834 & 0.004834 & 0.0423 \\
\hline & ${ }^{16} \mathrm{O}$ & 1.3224E-02 & 1.3228E-02 & 1.2056E-02 & \\
\hline & ${ }^{234} U$ & $<6.0 \mathrm{E}-08$ & $<6.0 \mathrm{E}-08$ & 2.5E-06 & \\
\hline & ${ }^{235} \mathrm{U}$ & $1.2832 \mathrm{E}-05$ & 1.2587E-05 & 2.6846E-04 & \\
\hline & ${ }^{238} \mathrm{U}$ & 6.3224E-03 & $6.2016 \mathrm{E}-03$ & 5.7595E-03 & \\
\hline & ${ }^{236} \mathrm{Pu}$ & $<3.0 \mathrm{E}-13$ & $<3.0 \mathrm{E}-11$ & & \\
\hline & ${ }^{238} \mathrm{Pu}$ & 5.5582E-08 & 6.0594E-06 & & \\
\hline & ${ }^{239} \mathrm{Pu}$ & 2.5998E-04 & 2.3251E-04 & & \\
\hline & ${ }^{240} \mathrm{Pu}$ & $1.6012 \mathrm{E}-05$ & $9.8145 \mathrm{E}-05$ & & \\
\hline & ${ }^{241} \mathrm{Pu}$ & 4.9403E-07 & 3.8298E-05 & & \\
\hline Fig. A.2. FA model & ${ }^{242} \mathrm{Pu}$ & 8.1995E-08 & 2.1056E-05 & & \\
\hline & ${ }^{241} \mathrm{Am}$ & $5.4891 \mathrm{E}-08$ & $5.1861 \mathrm{E}-06$ & & \\
\hline
\end{tabular}


Table A.5. Atom composition of structure materials in the cask for fresh fuel [atoms/(b cm)]

\begin{tabular}{|c|c|c|c|c|c|}
\hline & Zone & Region 1 & Region 2 & Region 3 & Region 4 \\
\hline & Material & Air & $\begin{array}{c}\text { Stainless } \\
\text { steel }\end{array}$ & Caoutchouc & $\begin{array}{c}\text { Stainless } \\
\text { steel }\end{array}$ \\
\hline & $\mathrm{R}, \mathrm{cm}$ & 12.6 & 13.4 & 20.4 & 21.0 \\
\hline & $\Delta \mathrm{R}, \mathrm{cm}$ & 12.6 & 0.8 & 7.0 & 0.6 \\
\hline & $\mathrm{H}$ & & 0.0001 & 0.05372 & \\
\hline & C & & & 0.01791 & 0.0001 \\
\hline & $\mathrm{N}$ & & & & \\
\hline & $\mathrm{O}$ & & & 0.00895 & \\
\hline & $\mathrm{Si}$ & & & 0.00895 & \\
\hline & $\mathrm{Cr}$ & & 0.01525 & & 0.01525 \\
\hline & $\mathrm{Fe}$ & & 0.06006 & & 0.06006 \\
\hline & $\mathrm{Ni}$ & & 0.00847 & & 0.00847 \\
\hline & $\mathrm{Ti}$ & & 0.00085 & & 0.00085 \\
\hline Fig. A.3. Cask for fresh fuel & & & & & \\
\hline
\end{tabular}




\section{A.4.4 Cask Model for Spent Fuel}

The cask model for spent fuel is based that which is typical for a transportation of 12 spent FA. It is a cylinder model shown in Fig. A.4. The geometric specifications and structure materials compositions are presented in Table A.6. The fuel volume fraction in Region 1 is equal to 0.128. The volume fraction of $12 \mathrm{FA}$ is equal to 0.423 . For calculation of fuel nuclide densities in Region 1 the calculated spent fuel composition (zone 1 of pin-cell) should be multiplied by the factor 0.128 .

Table A.6. Atom composition of structure materials in the cask for spent fuel [atoms/(b cm)]

\begin{tabular}{|c|c|c|c|}
\hline Region & 1 & 2 & 3 \\
\hline \hline Diameter, cm & 132 & 200 & 225 \\
\hline \hline $\mathrm{T}, \mathrm{K}$ & 523 & 300 & 300 \\
\hline \hline $\mathrm{Zr}$ & 0.002216 & & \\
\hline \hline $\mathrm{Fe}$ & 0.0027 & 0.061 & \\
\hline \hline $\mathrm{Cr}$ & 0.0007 & 0.016 & \\
\hline \hline $\mathrm{Ni}$ & 0.0004 & 0.008 & \\
\hline \hline $\mathrm{B}$ & 0.00029 & & \\
\hline \hline $\mathrm{O}$ & 0.0054 & & 0.026 \\
\hline \hline $\mathrm{C}$ & & & 0.014 \\
\hline $\mathrm{H}$ & & & 0.065 \\
\hline
\end{tabular}




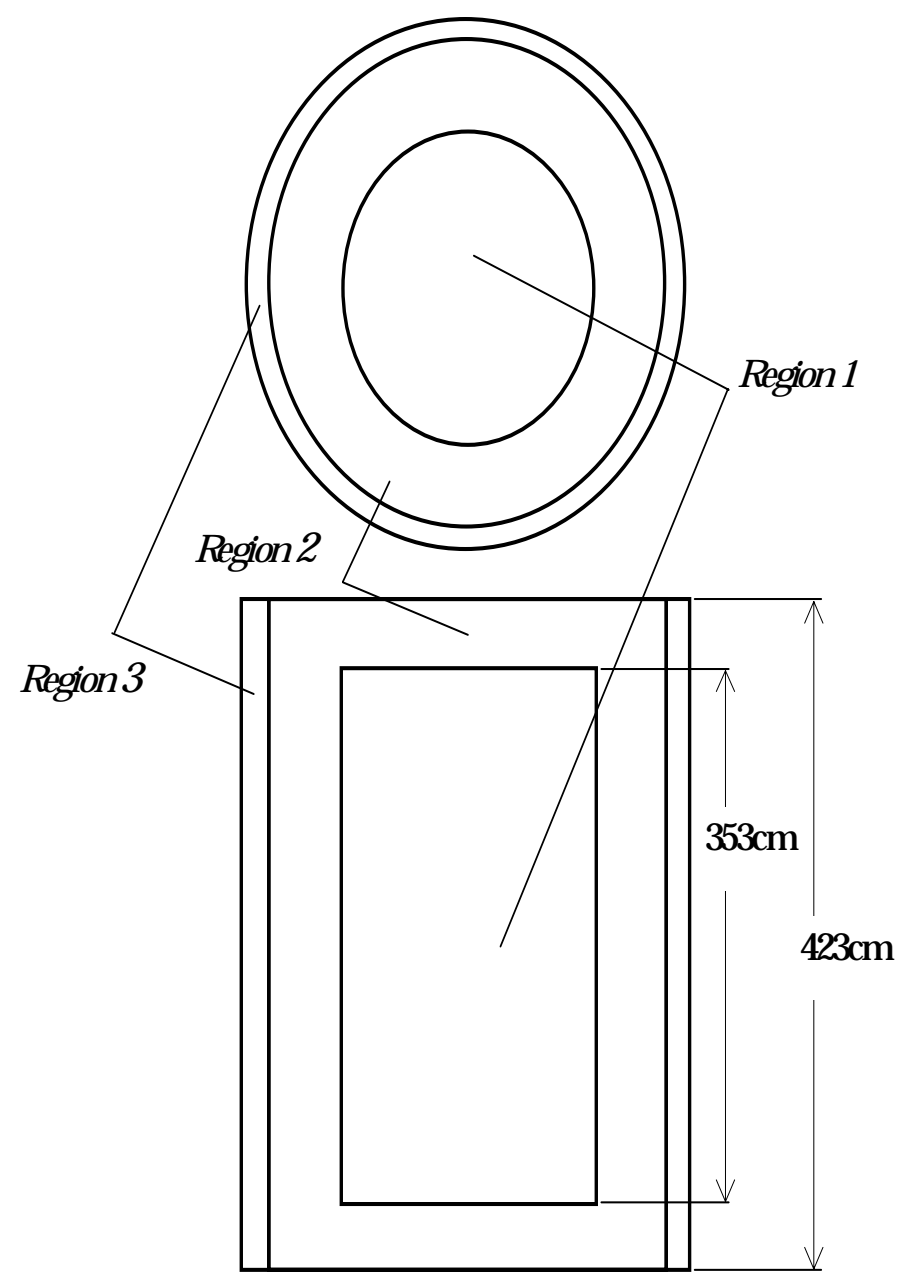

Fig. A.4. Cask geometry of spent FA. 


\section{A.5 Desired Results}

For the Task $\mathrm{I}$ the desired results are a dependence of criticality parameter $\mathrm{k}_{\text {eff }}$ via water density:

- Function $\mathrm{k}_{\text {eff }}(\gamma)$ for $\gamma\left(\mathrm{H}_{2} \mathrm{O}\right)=1.0,0.9,0.8,0.7,0.6,0.5,0.4,0.3,0.2,0.1,0.05,0.03$, 0.02 and 0.

For the Task II the desired results are shielding and radioactive characteristics of FA:

- Neutron source strength total and fractional by separate isotopes in used group structure.

- Gamma source strength in used group structure.

- Dose rates at distance from the surface of $\mathrm{FA}$ at $\mathrm{d}=0,0.5,1$ and 2 meter.

For the Task IIb fresh fuel calculations additionally should be presented:

- Heat generation [Wt/FA] - total and fractional by actinides associated with spontaneous fission and (alfa,n) reaction on oxygen [Wt/FA].

For the Task IIc spent fuel calculations additionally should be presented:

- Nuclide composition [g/FA].

- Activity of actinides and fission products [Bk/FA].

- Dose rates at distance from the surface of Cask at $\mathrm{d}=0,0.5,1$ and 2 meter with spent FA after disposition in a pool storage $\mathrm{t}=3$ year.

- Heat generation - total and fractionally for actinides, associated with spontaneous fission and (alfa,n) reaction on oxygen, and for fission products in spent fuel (one FA) via time of disposition $\mathrm{t}=3$ and 10 days, and $1,3,10,100$ years.

List of actinides includes isotopes from U-232 up to Cm-248: U-232 U-233 U-234 U-235 U-236 U-237 U-238 Np-237 Np-238 Np-239 Pu-236 Pu-238 Pu-239 Pu-240 Pu-241 Pu-242 Am-241 Am-242 Am-242m Am-243 Cm-242 Cm-243 Cm-244 Cm-245 Cm-246 Cm-247 Cm-248.

List of fission products is defined for the following nuclei: Kr-85 Sr-90 Zr-93 Zr-95 Nb-95m Nb-95

Ru-106 Ag-110m I-129 I-131 Xe-133 Cs-134 Cs-136 Cs-137 Ce-141 Ce-144 Nd-147 Pm-147 Eu-154 Eu-155.

\section{A.6 Contacts}

With questions contact, please, to Gennadi Manturov (SSC RF IPPE, Obninsk), e-mail: abbn@ippe.rssi.ru. 


\section{APPENDIX B \\ CALCULATION RESULTS FOR TASK I: CRITICALITY SAFETY STUDY IN STORAGE}




\section{APPENDIX B}

\section{CALCULATION RESULTS FOR TASK I: CRITICALITY SAFETY STUDY IN STORAGE}

\section{B.1 The Desired Results}

Benchmarks for criticality safety calculations simulating storage arrays of $\mathrm{UO}_{2}$ - and $\mathrm{MOX}$ - VVER - fuel assemblies have been defined in proposal /1/.

\section{Geometry Model and Materials}

In this task a study of criticality safety in fresh fuel storage is performed. It is assumed infinite pool storage of FA's filled with a cold water. The triangular pitch is $40 \mathrm{~cm}$. The geometric data for FA is given in Table B.1 and in Fig.B.1-B.2. Table B.2 contains fresh fuel compositions for MOX-W (4.2\%) weapons-grade and MOX-R (6.1\%) civil, reactor-grade plutonium, and UOX $(4.4 \%)$.

The temperature of FA for fresh condition is $\mathrm{T}=300 \mathrm{~K}$. The cold water composition at $\mathrm{T}=300 \mathrm{~K}$ and $\gamma\left(\mathrm{H}_{2} \mathrm{O}\right)=1 \mathrm{~g} / \mathrm{cm}^{3}$ is $[\mathrm{atom} /(\mathrm{b} \times \mathrm{cm})]: \mathrm{H}-0.06694 ; \mathrm{O}-0.03347$.

A hexagonal cell model with reflective boundaries was used for the infinite storage array of the fuel assemblies. The vertical length was set to $353 \mathrm{~cm}$ with reflective boundaries on bottom and top. 
Table B.1. General assembly data

\begin{tabular}{lc}
\hline \multicolumn{1}{c}{ Parameter } & Value \\
\hline \multicolumn{1}{c}{ Number of fuel pins } & 312 \\
Number of guide tubes & 18 \\
Number of instrumentation tubes & 1 \\
Pin pitch, cm & 1.275 \\
Fuel rods & \\
$\quad$ Pellet diameter, cm & 0.772 \\
Clad inside diameter, cm & 0.772 \\
Clad outside diameter, cm & 0.91 \\
Clad material & $\mathrm{Zr}$ \\
Active fuel length, cm & 353 \\
Guide tubes & 1.09 \\
Inside diameter, cm & 1.265 \\
Outside diameter, cm & $\mathrm{Zr}$ \\
Material & \\
Central instrumentation tube & 0.96 \\
Inside diameter, cm & 1.125 \\
Outside diameter, cm & $\mathrm{Zr}$ \\
Material
\end{tabular}

Table B.2. MOX and UOX fresh fuel compositions [atom/(b×cm)]

\begin{tabular}{|c|l|l|l|}
\hline Nuclides & \multicolumn{1}{|c|}{ UOX (4.4\%) } & \multicolumn{1}{|c|}{ MOX-W (4.2\%) } & \multicolumn{1}{|c|}{ MOX-R (6.1\%) } \\
\hline${ }^{16} \mathrm{O}$ & $3.9235 \mathrm{E}-02$ & $4.3036 \mathrm{E}-02$ & $4.3051 \mathrm{E}-02$ \\
\hline${ }^{234} \mathrm{U}$ & $8.0 \mathrm{E}-06$ & $2.0 \mathrm{E}-07$ & $2.0 \mathrm{E}-07$ \\
\hline${ }^{235} \mathrm{U}$ & $8.7370 \mathrm{E}-04$ & $4.1762 \mathrm{E}-05$ & $4.0964 \mathrm{E}-05$ \\
\hline${ }^{238} \mathrm{U}$ & $1.8744 \mathrm{E}-02$ & $2.0576 \mathrm{E}-02$ & $2.0183 \mathrm{E}-02$ \\
\hline${ }^{238} \mathrm{Pu}$ & & $1.8089 \mathrm{E}-07$ & $1.9720 \mathrm{E}-05$ \\
\hline${ }^{239} \mathrm{Pu}$ & & $8.4610 \mathrm{E}-04$ & $7.5671 \mathrm{E}-04$ \\
\hline${ }^{240} \mathrm{Pu}$ & & $5.2111 \mathrm{E}-05$ & $3.1941 \mathrm{E}-04$ \\
\hline${ }^{241} \mathrm{Pu}$ & & $1.6078 \mathrm{E}-06$ & $1.2464 \mathrm{E}-04$ \\
\hline${ }^{242} \mathrm{Pu}$ & & $2.6685 \mathrm{E}-07$ & $6.8527 \mathrm{E}-05$ \\
\hline${ }^{241} \mathrm{Am}$ & & $1.7864 \mathrm{E}-07$ & $1.6878 \mathrm{E}-05$ \\
\hline
\end{tabular}

${ }^{*}$ All $\mathrm{Pu}$. 


\section{Cladding.}

A uniform Zirconium composition 0.0423 atoms/(b×cm) is assumed.

The calculations are to be performed for each type of fuel to provide a dependence of criticality parameter keff via water density for $\gamma\left(\mathrm{H}_{2} \mathrm{O}\right)=1.0,0.9,0.8,0.7,0.6,0.5,0.4,0.3,0.2,0.1$, $0.05,0.03,0.02$ and $0\left[\mathrm{~g} / \mathrm{cm}^{3}\right]$.

\section{B.2 Short Description of the Used Methods}

\begin{tabular}{|c|c|c|}
\hline Participants & CODE LIBRARY & Organization \\
\hline T. Ivanova & ABBN93 & IPPE \\
\hline V. Vnukov & MMKFK ABBN78 & IPPE \\
\hline Ye. Rozhikhin & MCNP B-V/C & IPPE \\
\hline V. Koscheev & MCNP & IPPE \\
\hline M. Semenov & MCNP & IPPE \\
\hline S. Marin & MCUDAT & RRC KI \\
\hline G. Jerdev & ABBN93/S & IPPE \\
\hline
\end{tabular}

All calculations have been performed with Monte-Carlo method using different crosssection libraries.

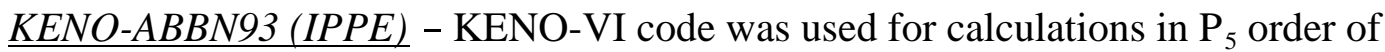
anisotropy with 299-group ABBN93 cross-section data set /2/. The resonance self-shielding effects were taken into account by using Bondarenko self-shielding factors. The thermalization effects were taken into account by using thermalized $\mathrm{P}_{0}$ and $\mathrm{P}_{1}$ multigroup scattering matrices in energy region below $4.65 \mathrm{eV}$.

MMKFK-ABBN78 (IPPE) - MMKFK code was used for calculations with 26-group ABBN78 cross-section data set /3/ and subgroup approximation in resonance region. The thermalization effects were taken into in energy region below $1 \mathrm{eV}$.

$\underline{M C N P-B V / C(I P P E)}$ - MCNP-4a code with continuous-energy cross-section library based on the ENDF/B-V data set. 
$\underline{M C N P-B V / S(I P P E)}$ - the same code and library as previous calculation but the subgroup approximation was used for ${ }^{235} \mathrm{U},{ }^{238} \mathrm{U}$ and ${ }^{239} \mathrm{Pu}$ in unresolved resonance region.

MCNP-BVI (IPPE) - MCNP-4a code with continuous-energy cross-section library based on the ENDF/B-VI (Release 2) data set.

$\underline{M C U-M C U D A T(R R C K I)}$ - MCU-RFFI/A code with DLC/MCUDAT-1.0 cross-section library.

Note:

1. The sizes of guide tubes and central instrumentation tube are different from sizes at table 1 ( Description of test).

Used sizes (at description): inside diameter - 1.10(1.09), outside diameter - 1.30(1.265). So the total area became larger than $17 \%$.

2. The ZR-alloy $(\mathrm{Zr}+\mathrm{Nb}+\mathrm{Hf})$ was used instead $\mathrm{ZR}(\rho=0.0423)$.

3. Diameter of fuel pellet is also different $-0.755(0.772)$, but atomic densities of fuel are the same.

As the result for UOX with density of water $1 \mathrm{~g} / \mathrm{cm}^{3}$ it leads to decrease of $\mathrm{k}_{\text {eff }}-0.6 \%$.

KENO-ABBN93/S (IPPE) - the same code and cross-sections as KENO-ABBN93 but the subgroup approximation was used for ${ }^{238} \mathrm{U}$ in resonance region.

\section{$\underline{\text { Results }}$}

All calculation results and its comparing are presented at Table B.3 - B.8 and Figure B.3

\section{-B.5.}

1. Pavlovitchev (RRC RF KI), A. Kalashnikov, G. Manturov (SSC RF IPPE). Description of Safety Analysis Computational Benchmark. IPPE.

2. G. Manturov, M. Nikolaev, A. Tsiboulia. ABBN-93 Group Data Library. Nuclear Data for Calculation of Neutron and Photon Radiation Fields. Vienna, IAEA, INDC(CCP)409/L,1997.

3. L. Abagyan et al. Gruppovye konstanti dlya rascheta reaktorov i zaschity. M., Energoizdat, 1981 (in Russia). 

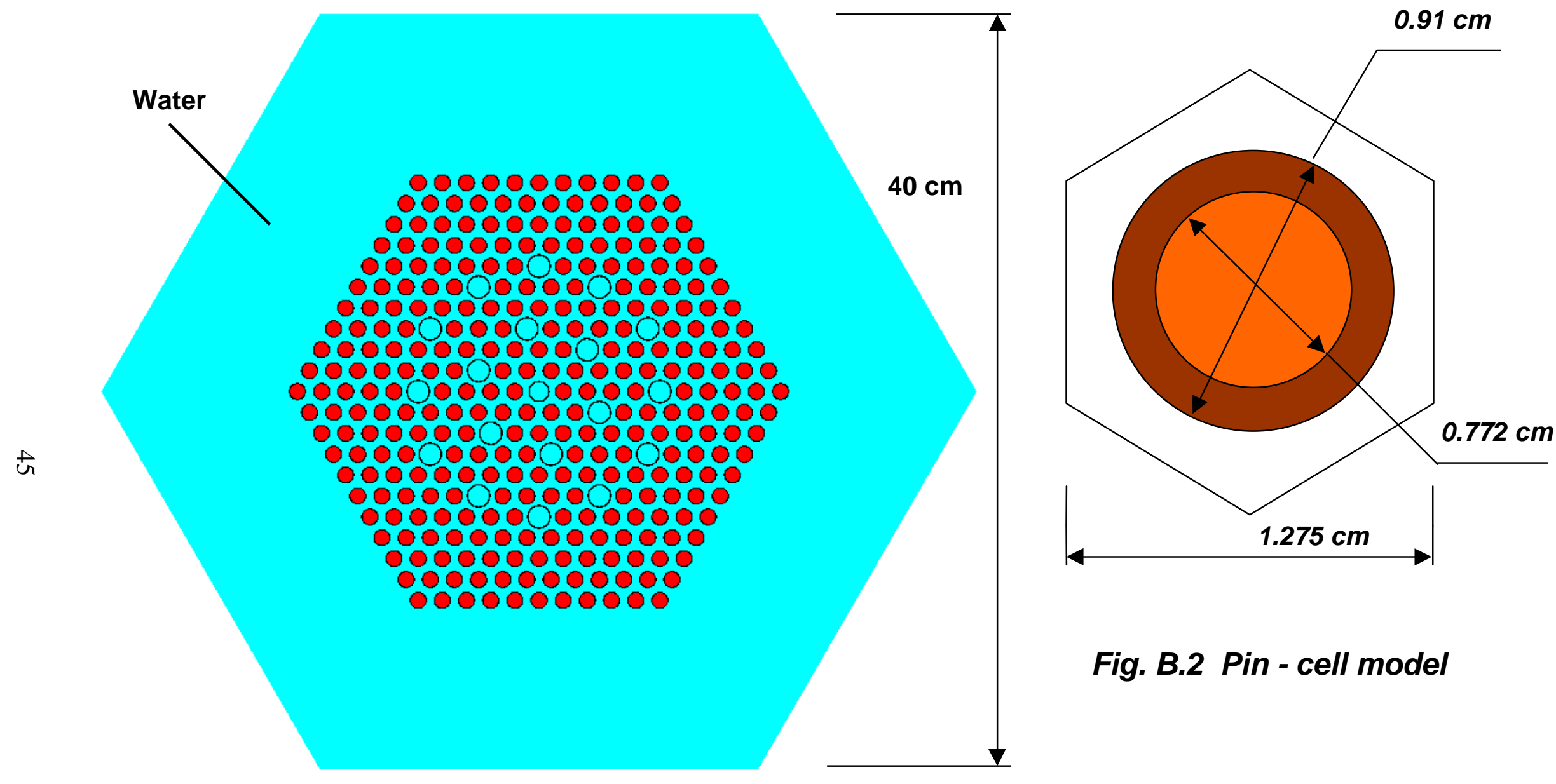

Fig. B.1 Calculation cell of storage

Fig. B.2 Pin - cell model 
Table B.3. K-inf for UOX fuel

\begin{tabular}{|c|c|c|c|c|c|c|c|}
\hline $\begin{array}{l}\text { CODE } \\
\text { LIBRARY } \\
\text { Organization } \\
\text { H2O density }\end{array}$ & $\begin{array}{c}\text { KENO } \\
\text { ABBN93 } \\
\text { IPPE }\end{array}$ & $\begin{array}{c}\text { MMKFK } \\
\text { ABBN78 } \\
\text { IPPE }\end{array}$ & $\begin{array}{l}\text { MCNP } \\
\text { B-V/C } \\
\text { IPPE }\end{array}$ & $\begin{array}{l}\text { MCNP } \\
\text { B-V/S } \\
\text { IPPE }\end{array}$ & $\begin{array}{c}\text { MCNP } \\
\text { B-VI } \\
\text { IPPE }\end{array}$ & $\begin{array}{c}\text { MCU } \\
\text { MCUDAT } \\
\text { RRC KI }\end{array}$ & $\begin{array}{c}\text { KENO } \\
\text { ABBN93/S } \\
\text { IPPE }\end{array}$ \\
\hline 0.00 & 0.6931 & 0.7060 & $0.6679 *$ & 0.6927 & 0.7000 & 0.6916 & 0.6921 \\
\hline 0.02 & 1.0382 & 1.0190 & 1.0067 & 1.0166 & 1.0171 & 1.0183 & 1.0325 \\
\hline 0.03 & 1.1400 & 1.1210 & 1.1140 & 1.1229 & 1.1223 & 1.1259 & 1.1389 \\
\hline 0.05 & 1.2798 & 1.2620 & 1.2531 & 1.2608 & 1.2578 & 1.2645 & 1.2746 \\
\hline 0.10 & 1.3895 & 1.3780 & 1.3757 & 1.3781 & 1.3769 & 1.3791 & 1.3881 \\
\hline 0.20 & 1.3112 & 1.3100 & 1.3048 & 1.3065 & 1.3035 & 1.3071 & 1.3115 \\
\hline 0.30 & 1.1503 & 1.1560 & 1.1515 & 1.1530 & 1.1496 & 1.1559 & 1.1571 \\
\hline 0.40 & 1.0234 & 1.0290 & 1.0217 & 1.0239 & 1.0191 & 1.0246 & 1.0267 \\
\hline 0.50 & 0.9449 & 0.9410 & 0.9386 & 0.9394 & 0.9363 & 0.9447 & 0.9426 \\
\hline 0.60 & 0.8996 & 0.9040 & 0.8969 & 0.8964 & 0.8926 & $0.9140 *$ & 0.9005 \\
\hline 0.70 & 0.8876 & 0.8930 & 0.8828 & 0.8842 & 0.8798 & 0.8859 & 0.8888 \\
\hline 0.80 & 0.8937 & 0.8960 & 0.8899 & 0.8911 & 0.8864 & 0.8891 & 0.8954 \\
\hline 0.90 & 0.9109 & 0.9150 & 0.9077 & 0.9074 & 0.9020 & 0.9063 & 0.9119 \\
\hline 1.00 & 0.9328 & 0.9340 & 0.9291 & 0.9295 & 0.9250 & 0.9266 & 0.9344 \\
\hline
\end{tabular}

${ }^{*}$ Not used for calculation Mean 
Table B.4. K-inf for UOX fuel

\begin{tabular}{|l|l|l|l|l|l|l|l|l|}
\hline $\begin{array}{l}\text { CODE } \\
\text { LIBRARY } \\
\text { Organization } \\
\text { H2O density }\end{array}$ & $\begin{array}{c}\text { MEAN } \\
\text { Std. Dev.,\%) }\end{array}$ & $\begin{array}{c}\text { KENO } \\
\text { ABBN93 } \\
\text { IPPE }\end{array}$ & $\begin{array}{c}\text { MMKFK } \\
\text { ABBN78 } \\
\text { IPPE }\end{array}$ & $\begin{array}{c}\text { MCNP } \\
\text { B-V/C } \\
\text { IPPE }\end{array}$ & $\begin{array}{c}\text { MCNP } \\
\text { B-V/S } \\
\text { IPPE }\end{array}$ & $\begin{array}{c}\text { MCNP } \\
\text { B-VI } \\
\text { IPPE }\end{array}$ & $\begin{array}{c}\text { MCU } \\
\text { MCUDAT } \\
\text { RRC KI }\end{array}$ & $\begin{array}{c}\text { KENB } \\
\text { ABN93/S } \\
\text { IPPE }\end{array}$ \\
\hline $\mathbf{0}$ & $0.6963(\mathbf{0 . 5 4})$ & -0.47 & 1.39 & - & -0.52 & 0.53 & -0.68 & -0.61 \\
\hline $\mathbf{0 . 0 2}$ & $1.0181(\mathbf{1 . 3 0})$ & 1.98 & 0.09 & -1.12 & -0.15 & -0.10 & 0.02 & 1.42 \\
\hline $\mathbf{0 . 0 3}$ & $1.1231(\mathbf{1 . 1 7})$ & 1.51 & -0.18 & -0.81 & -0.01 & -0.07 & 0.25 & 1.41 \\
\hline $\mathbf{0 . 0 5}$ & $1.2609(\mathbf{1 . 0 9})$ & 1.50 & 0.09 & -0.62 & -0.01 & -0.25 & 0.28 & 1.09 \\
\hline $\mathbf{0 . 1 0}$ & $1.3788(\mathbf{0 . 3 9})$ & 0.77 & -0.06 & -0.23 & -0.05 & -0.14 & 0.02 & 0.67 \\
\hline $\mathbf{0 . 2 0}$ & $1.3070(\mathbf{0 . 2 8})$ & 0.32 & 0.23 & -0.17 & -0.04 & -0.27 & 0.01 & 0.34 \\
\hline $\mathbf{0 . 3 0}$ & $1.1532(\mathbf{0 . 2 4})$ & -0.25 & 0.24 & -0.15 & -0.02 & -0.31 & 0.23 & 0.34 \\
\hline $\mathbf{0 . 4 0}$ & $1.0239(\mathbf{0 . 2 9})$ & -0.05 & 0.50 & -0.22 & 0 & -0.47 & 0.07 & 0.27 \\
\hline $\mathbf{0 . 5 0}$ & $0.9409(\mathbf{0 . 3 2})$ & 0.42 & 0.01 & -0.25 & -0.16 & -0.49 & 0.40 & 0.18 \\
\hline $\mathbf{0 . 6 0}$ & $0.9004(\mathbf{0 . 4 0})$ & -0.08 & 0.41 & -0.38 & -0.44 & -0.86 & - & 0.02 \\
\hline $\mathbf{0 . 7 0}$ & $0.8857(\mathbf{0 . 4 6})$ & 0.22 & 0.83 & -0.33 & -0.17 & -0.66 & 0.02 & 0.35 \\
\hline $\mathbf{0 . 8 0}$ & $0.8915(\mathbf{0 . 3 7})$ & 0.25 & 0.51 & -0.18 & -0.04 & -0.57 & -0.27 & 0.44 \\
\hline $\mathbf{0 . 9 0}$ & $0.9084(\mathbf{0 . 4 4})$ & 0.27 & 0.73 & -0.08 & -0.11 & -0.71 & -0.23 & 0.38 \\
\hline $\mathbf{1 . 0 0}$ & $0.9298(\mathbf{0 . 3 8})$ & 0.32 & 0.45 & -0.08 & -0.04 & -0.52 & -0.35 & 0.49 \\
\hline
\end{tabular}

*) $\left(K_{\text {eff,i }}-\right.$ Mean $) * 100 \% /$ Mean 
Fig. B.3. UOX fuel

\section{K-inf Average}
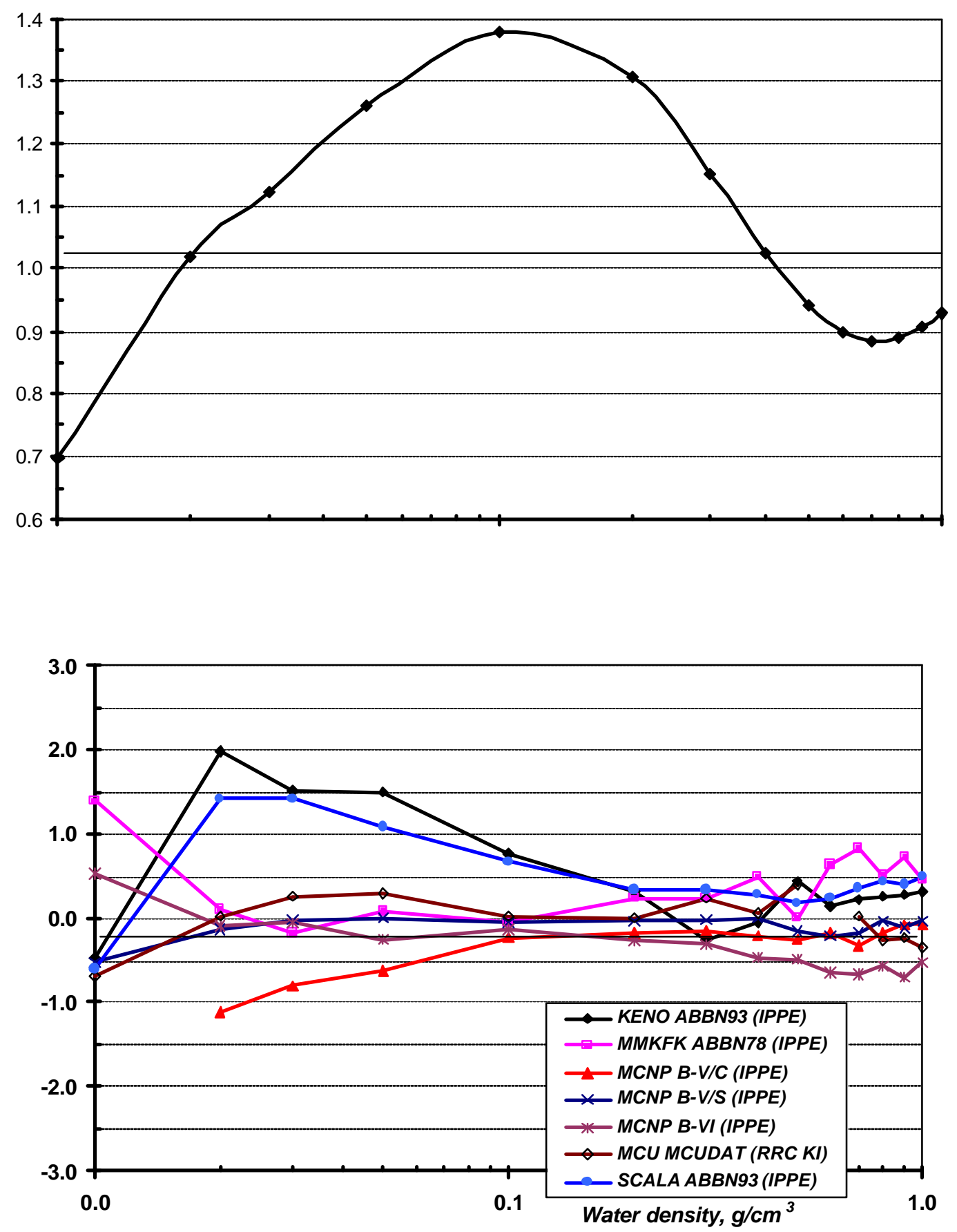

Percent Difference Relative to Mean 
Table B.5. K-inf for MOX-W fuel

\begin{tabular}{|c|c|c|c|c|c|c|c|}
\hline $\begin{array}{l}\text { CODE } \\
\text { LIBRARY } \\
\begin{array}{l}\text { Organization } \\
\text { H2O density }\end{array}\end{array}$ & $\begin{array}{c}\text { KENO } \\
\text { IPPE }\end{array}$ & $\begin{array}{c}\text { MMKFK } \\
\text { ABBN78 } \\
\text { IPPE }\end{array}$ & $\begin{array}{c}\text { MCNP } \\
\text { B-V/C } \\
\text { IPPE }\end{array}$ & $\begin{array}{c}\text { MCNP } \\
\text { B-V/S } \\
\text { IPPE }\end{array}$ & $\begin{array}{c}\text { MCNP } \\
\text { B-VI } \\
\text { IPPE }\end{array}$ & $\begin{array}{c}\text { MCU } \\
\text { MCUDAT } \\
\text { RRC KI }\end{array}$ & $\begin{array}{c}\text { KENO } \\
\text { ABBN93/S } \\
\text { IPPE }\end{array}$ \\
\hline 0 & 0.7238 & 0.7280 & $0.7098 *$ & 0.7272 & 0.7310 & 0.7148 & 0.7230 \\
\hline 0,02 & 0.9795 & 0.9510 & 0.9638 & 0.9719 & 0.9708 & 0.9761 & 0.9780 \\
\hline 0,03 & 1.0688 & 1.0360 & 1.0519 & 1.0599 & 1.0575 & 1.0631 & 1.0632 \\
\hline 0,05 & 1.1911 & 1.1700 & 1.1800 & 1.1869 & 1.1821 & 1.1886 & 1.1862 \\
\hline 0,1 & 1.3237 & 1.3190 & 1.3201 & 1.3237 & 1.3178 & 1.3193 & 1.3181 \\
\hline 0,2 & 1.2849 & 1.2960 & 1.2919 & 1.2939 & 1.2837 & 1.2845 & 1.2829 \\
\hline 0,3 & 1.1501 & 1.1580 & 1.1571 & 1.1568 & 1.1491 & 1.1464 & 1.1479 \\
\hline 0,4 & 1.0215 & 1.0330 & 1.0293 & 1.0319 & 1.0229 & $1.0525 *$ & 1.0234 \\
\hline 0,5 & 0.9369 & 0.9500 & 0.9447 & 0.9449 & 0.9373 & 0.9402 & 0.9428 \\
\hline 0,6 & 0.8939 & 0.9080 & 0.8999 & 0.8994 & 0.8917 & $0.9057 *$ & 0.8960 \\
\hline 0,7 & 0.8791 & 0.8960 & 0.8836 & 0.8847 & 0.8758 & 0.8787 & 0.8815 \\
\hline 0,8 & 0.8850 & 0.8980 & 0.8887 & 0.8885 & 0.8815 & 0.8836 & 0.8859 \\
\hline 0,9 & 0.9016 & 0.9150 & 0.9036 & 0.9043 & 0.8984 & 0.9017 & 0.9048 \\
\hline 1 & 0.9232 & 0.9410 & 0.9257 & 0.9277 & 0.9206 & 0.9230 & 0.9267 \\
\hline
\end{tabular}

${ }^{*}$ Not used for calculation Mean 
Table B.6. Percent difference relative to mean (MOX-W fuel) *

\begin{tabular}{|c|c|c|c|r|r|r|r|r|}
\hline $\begin{array}{l}\text { CODE } \\
\text { LIBRARY } \\
\begin{array}{l}\text { Organization } \\
\text { H2O density }\end{array}\end{array}$ & $\begin{array}{c}\text { MEAN } \\
\text { (Std. Dev.,\%) }\end{array}$ & $\begin{array}{c}\text { KENO } \\
\text { ABBN93 } \\
\text { IPPE }\end{array}$ & $\begin{array}{c}\text { MMKFK } \\
\text { ABBN78 } \\
\text { IPPE }\end{array}$ & $\begin{array}{c}\text { MCNP } \\
\text { B-V/C } \\
\text { IPPE }\end{array}$ & $\begin{array}{c}\text { MCNP } \\
\text { B-V/S } \\
\text { IPPE }\end{array}$ & $\begin{array}{c}\text { MCNP } \\
\text { B-VI } \\
\text { IPPE }\end{array}$ & $\begin{array}{c}\text { MCU } \\
\text { MCUDAT } \\
\text { RRC KI }\end{array}$ & $\begin{array}{c}\text { KENO } \\
\text { ABBN93/S } \\
\text { IPPE }\end{array}$ \\
\hline $\mathbf{0}$ & $0.7248(\mathbf{0 . 7 2})$ & -0.14 & 0.44 & - & 0.33 & 0.86 & -1.38 & -0.25 \\
\hline $\mathbf{0 . 0 2}$ & $0.9693(\mathbf{0 . 9 8})$ & 1.05 & -1.89 & -0.57 & 0.27 & 0.15 & 0.70 & 0.90 \\
\hline $\mathbf{0 . 0 3}$ & $1.0562(\mathbf{0 . 9 8})$ & 1.20 & -1.91 & -0.40 & 0.36 & 0.13 & 0.66 & 0.67 \\
\hline $\mathbf{0 . 0 5}$ & $1.1823(\mathbf{0 . 6 3})$ & 0.75 & -1.04 & -0.19 & 0.39 & -0.01 & 0.53 & 0.33 \\
\hline $\mathbf{0 . 1}$ & $1.3192(\mathbf{0 . 2 8})$ & 0.34 & -0.02 & 0.07 & 0.34 & -0.11 & 0.01 & -0.08 \\
\hline $\mathbf{0 . 2}$ & $1.2874(\mathbf{0 . 4 3})$ & -0.20 & 0.67 & 0.35 & 0.50 & -0.29 & -0.23 & -0.35 \\
\hline $\mathbf{0 . 3}$ & $1.1518(\mathbf{0 . 4 1})$ & -0.15 & 0.54 & 0.46 & 0.43 & -0.23 & -0.47 & -0.34 \\
\hline $\mathbf{0 . 4}$ & $1.0264(\mathbf{0 . 4 8})$ & -0.47 & 0.65 & 0.29 & 0.54 & -0.34 & - & -0.29 \\
\hline $\mathbf{0 . 5}$ & $0.9419(\mathbf{0 . 4 8})$ & -0.53 & 0.86 & 0.30 & 0.32 & -0.49 & -0.18 & 0.09 \\
\hline $\mathbf{0 . 6}$ & $0.8973(\mathbf{0 . 6 4})$ & -0.38 & 1.19 & 0.29 & 0.24 & -0.62 & - & -0.14 \\
\hline $\mathbf{0 . 7}$ & $0.8822(\mathbf{0 . 7 1})$ & -0.35 & 1.56 & 0.16 & 0.28 & -0.73 & -0.40 & -0.08 \\
\hline $\mathbf{0 . 8}$ & $0.8864(\mathbf{0 . 6 3})$ & -0.16 & 1.31 & 0.26 & 0.23 & -0.56 & -0.32 & -0.06 \\
\hline $\mathbf{0 . 9}$ & $0.9026(\mathbf{0 . 7 4})$ & -0.11 & 1.38 & 0.11 & 0.19 & -0.46 & -0.10 & 0.25 \\
\hline $\mathbf{1}$ & $0.9258(\mathbf{0 . 7 3})$ & -0.28 & 1.64 & -0.01 & 0.20 & -0.56 & -0.31 & 0.09 \\
\hline
\end{tabular}

$*\left(K_{e f f, i}-\right.$ Mean $) * 100 \% / M e a n$ 
Fig. B.4. MOX - W fuel

\section{K-inf Average}

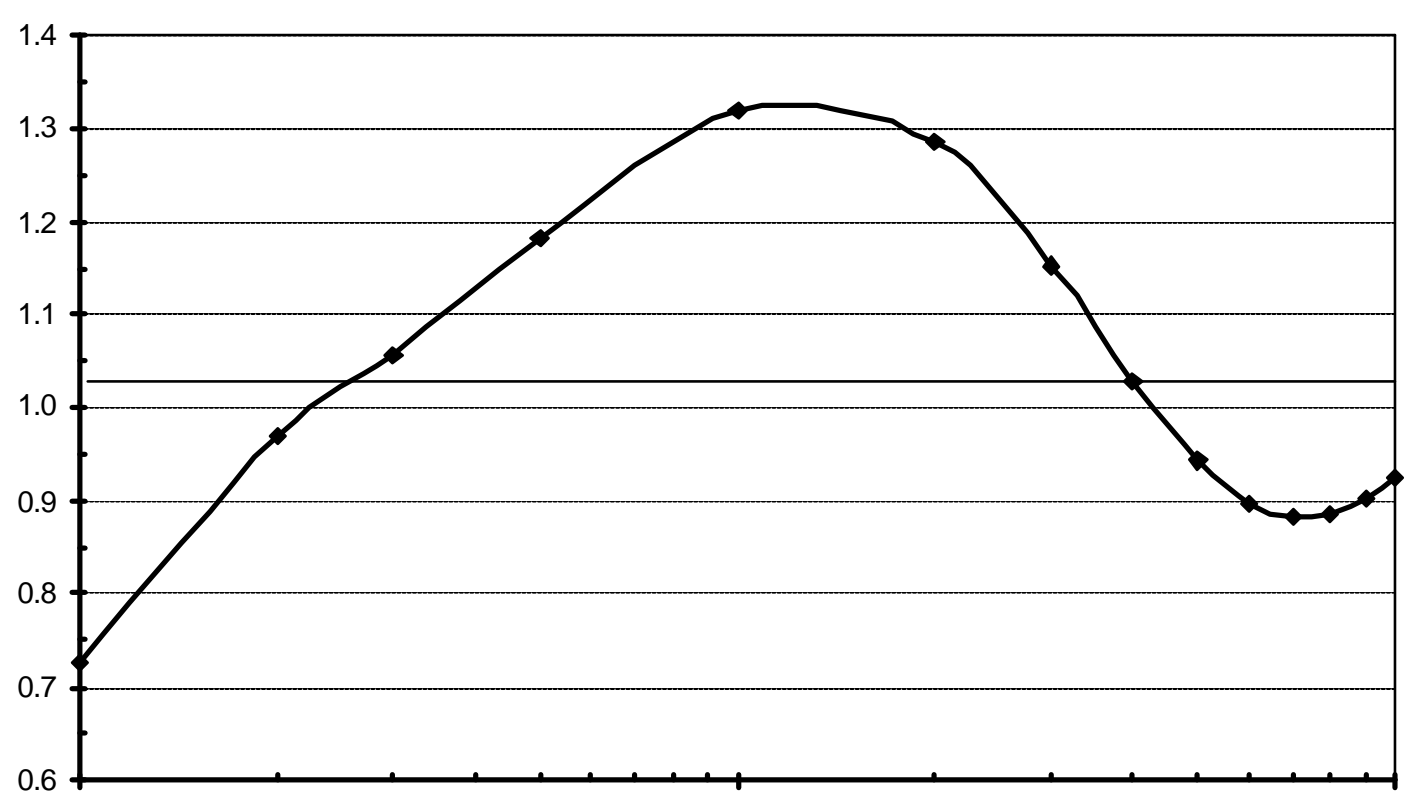

Percent Difference Relative to Mean

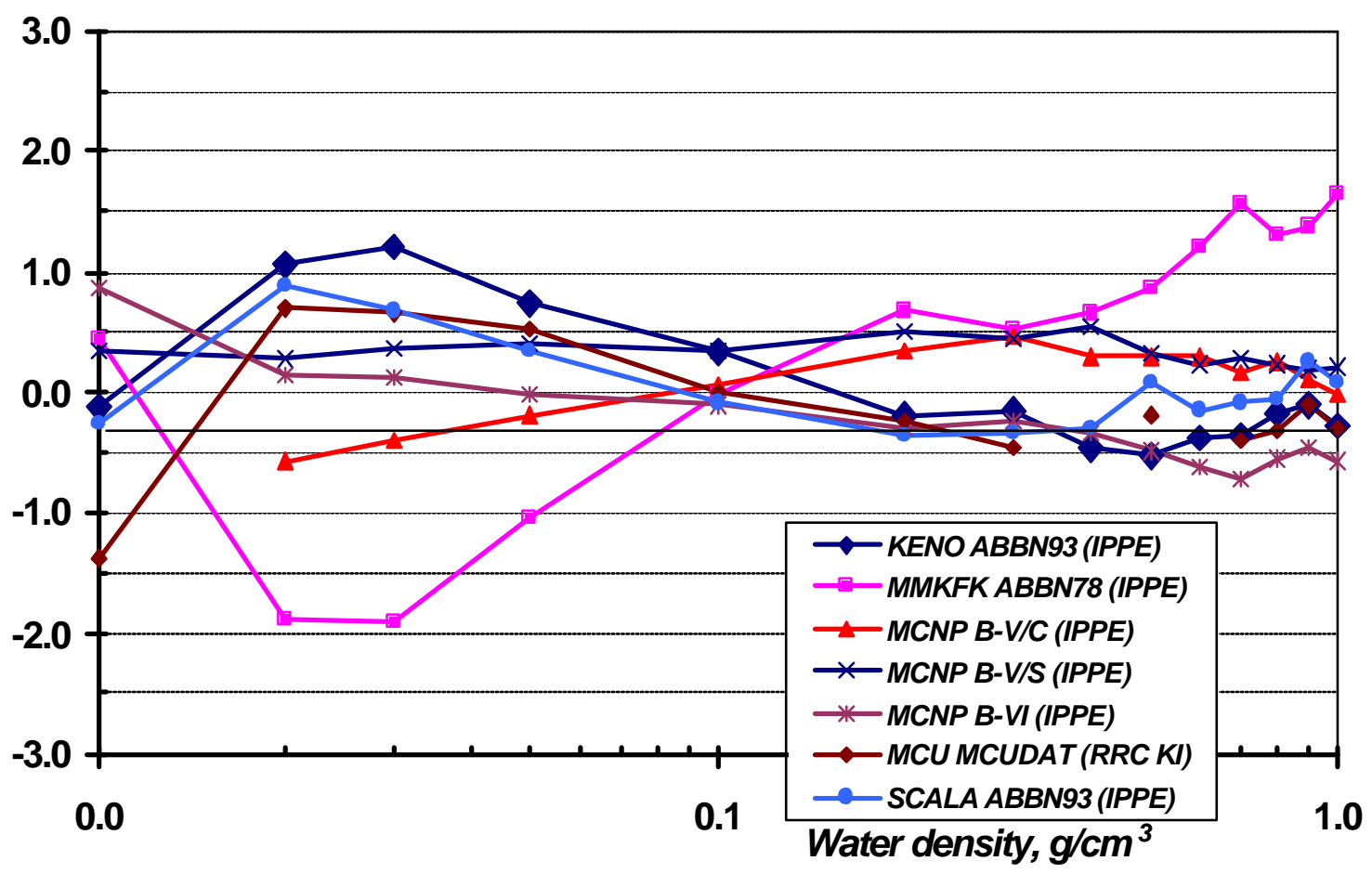


Table B.7. K-inf for MOX-R fuel

\begin{tabular}{|l|c|c|c|}
\hline $\begin{array}{l}\text { CODE } \\
\text { LIBRARY } \\
\text { Organization } \\
\text { H2O density }\end{array}$ & $\begin{array}{c}\text { KENO } \\
\text { ABBN93 } \\
\text { IPPE }\end{array}$ & $\begin{array}{c}\text { MMKFK } \\
\text { ABBN78 } \\
\text { IPPE }\end{array}$ & $\begin{array}{c}\text { MCU } \\
\text { MCUDAT } \\
\text { RRC KI }\end{array}$ \\
\hline $\mathbf{0 . 0 0}$ & 0.7862 & 0.7930 & 0.7794 \\
\hline $\mathbf{0 . 0 2}$ & 0.9623 & 0.9400 & 0.9537 \\
\hline \hline $\mathbf{0 . 0 3}$ & 1.0168 & 0.9980 & 1.0081 \\
\hline \hline $\mathbf{0 . 0 5}$ & 1.1038 & 1.0780 & 1.0973 \\
\hline \hline $\mathbf{0 . 1 0}$ & 1.2100 & 1.1980 & 1.2087 \\
\hline $\mathbf{0 . 2 0}$ & 1.1848 & 1.1780 & 1.1835 \\
\hline \hline $\mathbf{0 . 3 0}$ & 1.0640 & 1.0580 & 1.0596 \\
\hline \hline $\mathbf{0 . 4 0}$ & 0.9459 & 0.9440 & 0.9430 \\
\hline \hline $\mathbf{0 . 5 0}$ & 0.8647 & 0.8580 & 0.8638 \\
\hline \hline $\mathbf{0 . 6 0}$ & 0.8215 & 0.8180 & $0.8270 *$ \\
\hline \hline $\mathbf{0 . 7 0}$ & 0.8047 & 0.7960 & 0.8011 \\
\hline $\mathbf{0 . 8 0}$ & 0.8054 & 0.8030 & 0.8029 \\
\hline \hline $\mathbf{0 . 9 0}$ & 0.8197 & 0.8200 & 0.8187 \\
\hline \hline $\mathbf{1 . 0 0}$ & 0.8404 & 0.8390 & 0.8380 \\
\hline
\end{tabular}

* Not used for calculation Mean 
Table B.8. Percent difference relative to mean (MOX-R fuel) *

\begin{tabular}{|c|l|l|l|c|}
\hline $\begin{array}{l}\text { CODE } \\
\text { LIBRARY } \\
\text { Organization } \\
\mathrm{H}_{2} \text { O density }\end{array}$ & $\begin{array}{c}\text { MEAN } \\
\text { (Std. Dev.,\%) }\end{array}$ & $\begin{array}{c}\text { KENO } \\
\text { ABBN93 } \\
\text { IPPE }\end{array}$ & $\begin{array}{c}\text { MMKFK } \\
\text { ABBN78 } \\
\text { IPPE }\end{array}$ & $\begin{array}{c}\text { MCU } \\
\text { MCUDAT } \\
\text { RRC KI }\end{array}$ \\
\hline $\mathbf{0 . 0 0}$ & $0.7874(\mathbf{0 . 7 6})$ & -0.15 & 0.72 & -1.01 \\
\hline $\mathbf{0 . 0 2}$ & $0.9508(\mathbf{1 . 0 0})$ & 1.21 & -1.13 & 0.31 \\
\hline $\mathbf{0 . 0 3}$ & $1.0061(\mathbf{0 . 8 2})$ & 1.07 & -0.80 & 0.20 \\
\hline $\mathbf{0 . 0 5}$ & $1.0921(\mathbf{1 . 0 2})$ & 1.08 & -1.29 & 0.48 \\
\hline $\mathbf{0 . 1 0}$ & $1.2046(\mathbf{0 . 4 7})$ & 0.45 & -0.54 & 0.34 \\
\hline $\mathbf{0 . 2 0}$ & $1.1830(\mathbf{0 . 2 9})$ & 0.16 & -0.42 & 0.05 \\
\hline $\mathbf{0 . 3 0}$ & $1.0611(\mathbf{0 . 2 6})$ & 0.28 & -0.29 & -0.14 \\
\hline $\mathbf{0 . 4 0}$ & $0.9446(\mathbf{0 . 1 4})$ & 0.14 & -0.06 & -0.16 \\
\hline $\mathbf{0 . 5 0}$ & $0.8632(\mathbf{0 . 4 2})$ & 0.18 & -0.60 & 0.08 \\
\hline $\mathbf{0 . 6 0}$ & $0.8192(\mathbf{0 . 2 4})$ & 0.28 & -0.14 & - \\
\hline $\mathbf{0 . 7 0}$ & $0.8008(\mathbf{0 . 4 5})$ & 0.48 & -0.60 & 0.03 \\
\hline $\mathbf{0 . 8 0}$ & $0.8031(\mathbf{0 . 2 1})$ & 0.28 & -0.02 & -0.03 \\
\hline $\mathbf{0 . 9 0}$ & $0.8180(\mathbf{0 . 3 8})$ & 0.21 & 0.25 & 0.09 \\
\hline $\mathbf{1 . 0 0}$ & $0.8385(\mathbf{0 . 2 0})$ & 0.23 & 0.07 & -0.05 \\
\hline
\end{tabular}

${ }^{*}\left(K_{\text {effi, }}-\right.$ Mean $) * 100 \% /$ Mean 
Fig. B.5. MOX - R fuel

K-inf Average

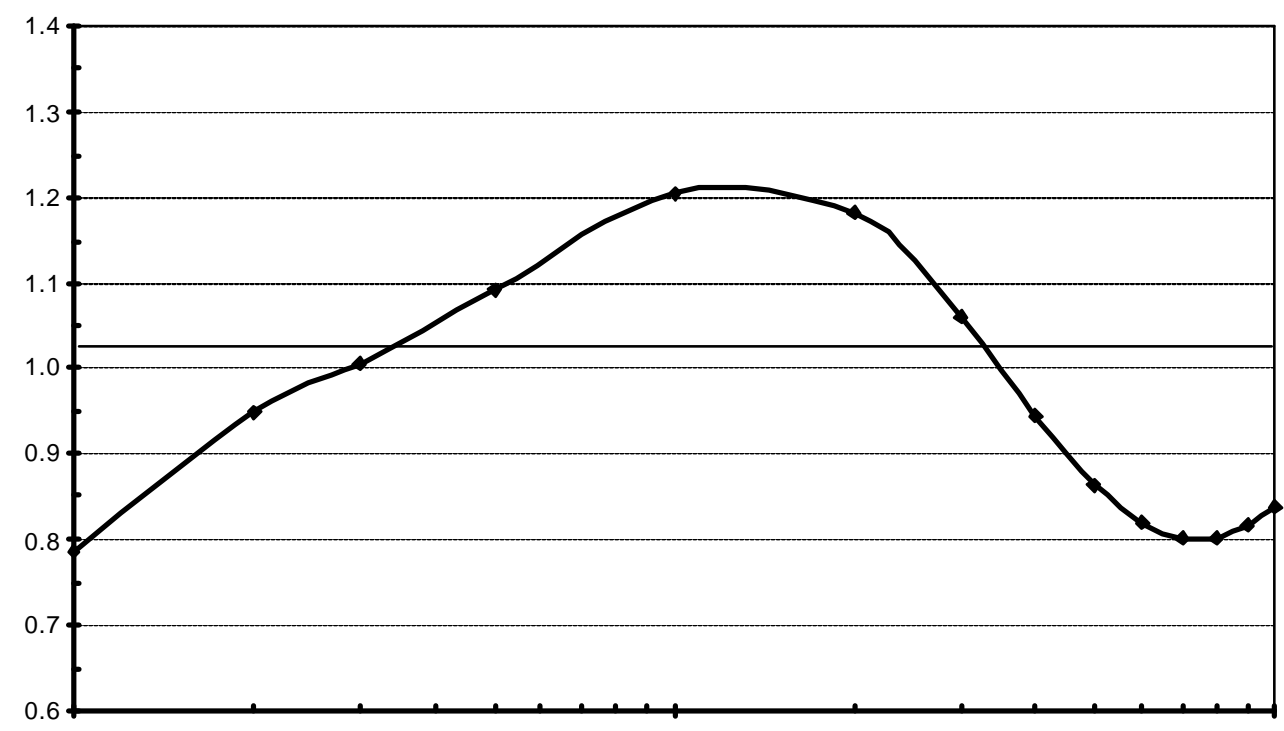

Percent Difference Relative to Mean

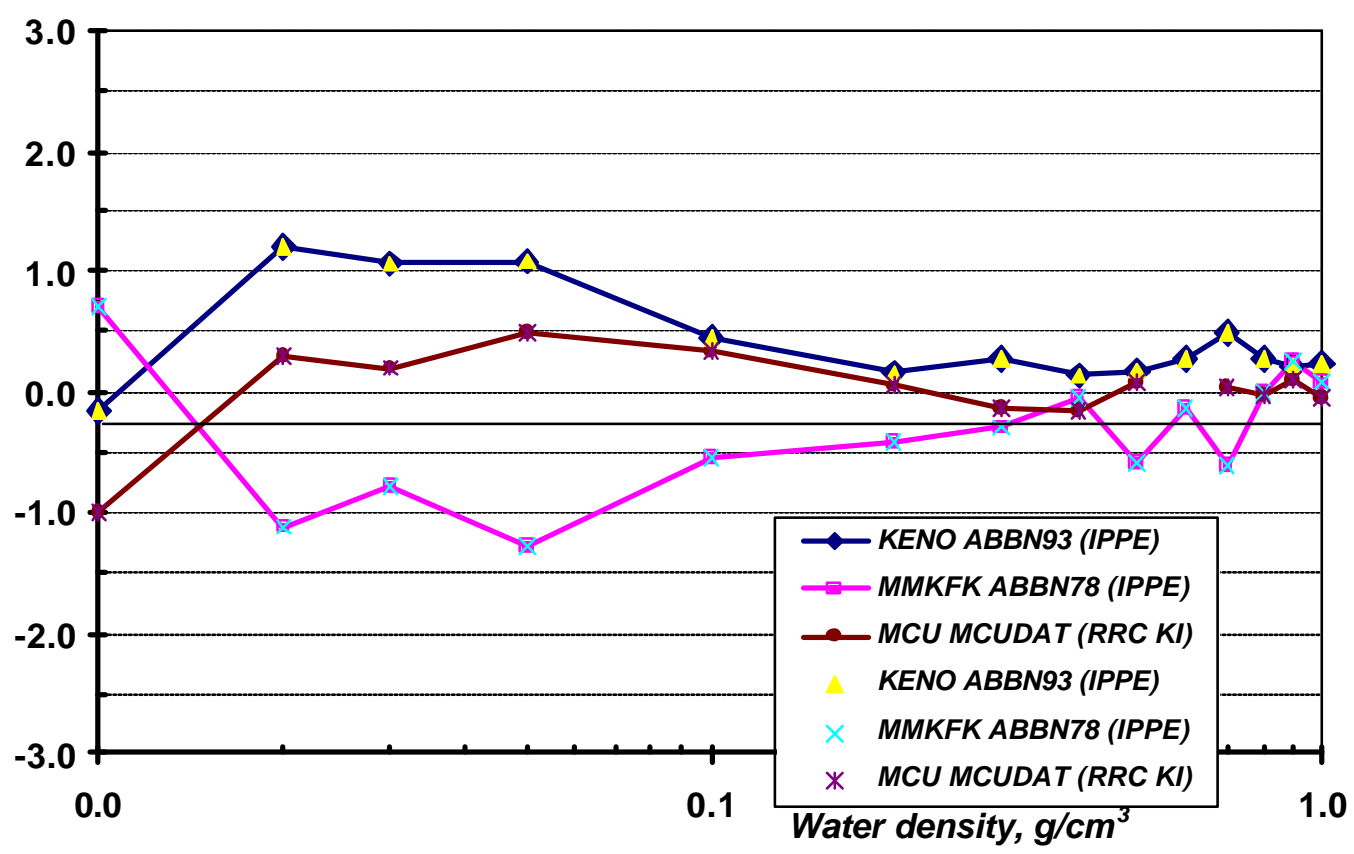


Fig. B.6. K-inf average

K-inf Average

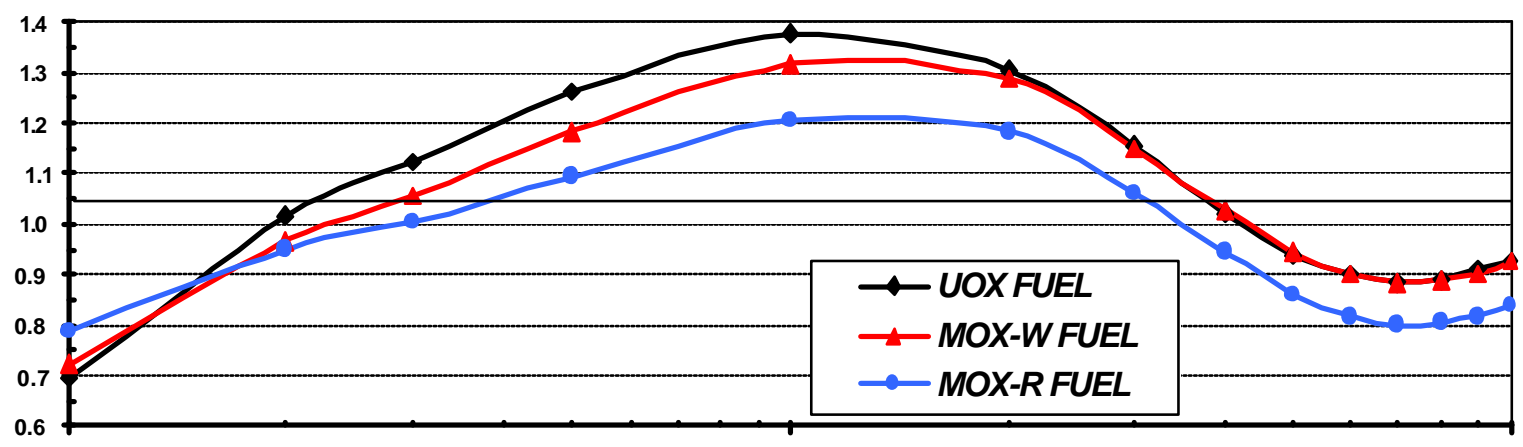

(MOX-W - -UOX)/UOX

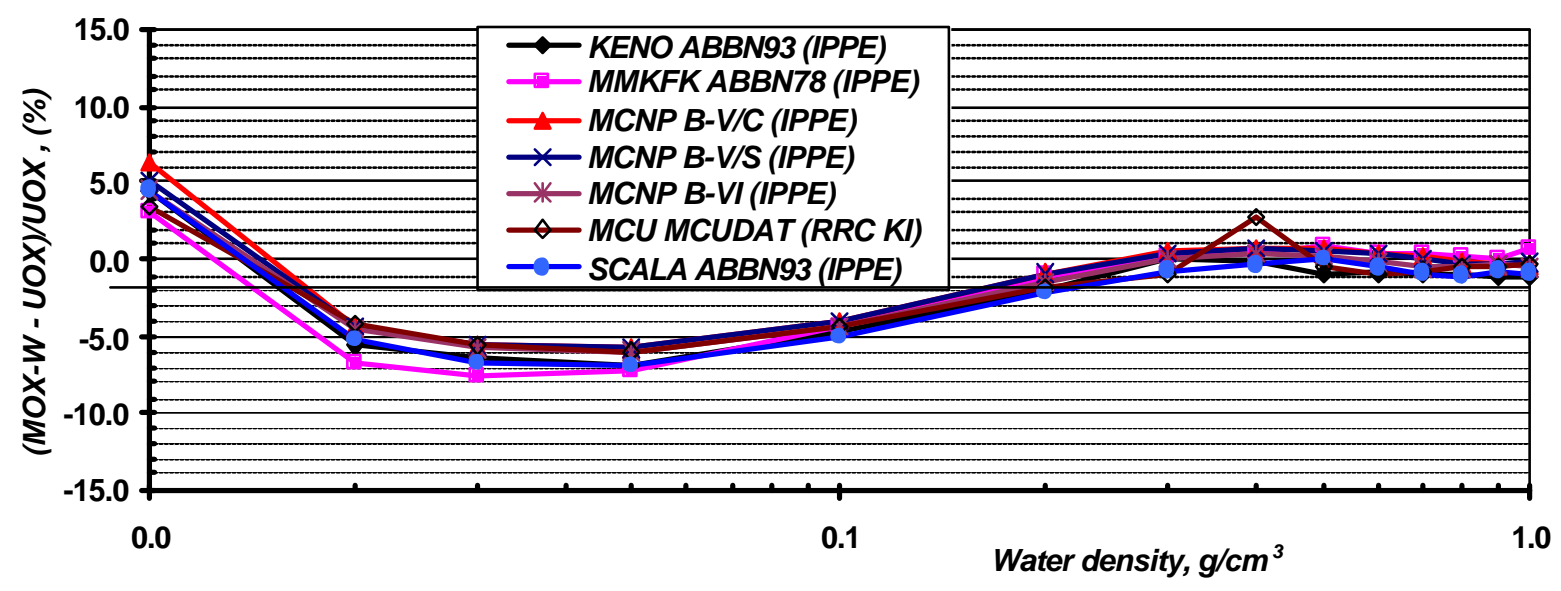

(MOX-R - -UOX)/UOX

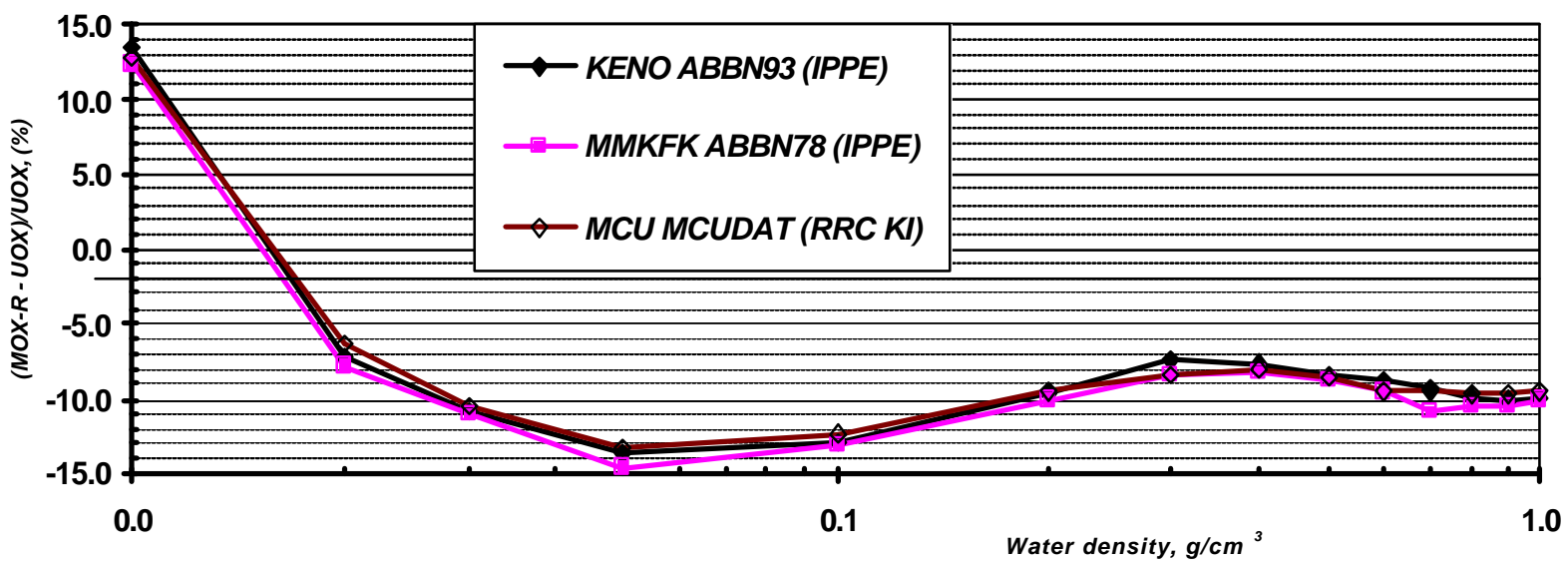





\section{APPENDIX C}

CALCULATION RESULTS FOR TASKS IIa AND IIb:

SHIELDING AND HEAT GENERATION STUDY FOR FRESH FUEL 


\section{APPENDIX C}

\section{CALCULATION RESULTS FOR TASK IIa AND TASK IIb: SHIELDING AND HEAT GENERATION STUDY FOR FRESH FUEL}

\section{C.1 The Desired Results}

In this task a study of shielding and radioactive characteristics of FAs with fresh fuel at a transportation is performed: the Task IIa - without a container and the Task IIb - with a Cask.

In this task a study of criticality safety under storing of fresh fuel is performed.

It is assumed a model of a dry assembly of fresh fuel with geometric specifications given in Table A.1 and in Fig. A.1 of the Benchmark Description. The temperature of FA for fresh conditions is T=300K. No water is presented. The composition of fresh fuel and the description of the Cask model are given in Tables A.4 and A.5 but also in Figs. A.2 and A.3 of the Description.

For each type of fuel should be calculated:

- Neutron source strength: total and fractional by separate isotopes and spectrum in a used group structure.

- Gamma source strength: total and spectrum in a used group structure.

- Dose rates at the distance from the surface of the FA or the Cask equal to 0, 0.5, 1 and 2 meter.

- Heat generation in the case of the Cask: total and fractional by actinides.

\section{C.2 Short Description of the Used Methods}

Participated results:

\begin{tabular}{|l|l|}
\hline Participants & ID \\
\hline \hline CARE + ANISN + ABBN-93 & IPPE-K \\
\hline ORIGEN + TWODANT + ABBN-93 & IPPE-Z \\
\hline \hline ANISN + CASK & IPPE-L \\
\hline
\end{tabular}

\section{IPPE-K (A. Kotchetkov, G. Khohlov)}

The sources of neutron and gamma emission were computed with CARE code [1] developed at IPPE. It calculates isotope compositions of actinides and fission products during reactor operating and after shutdown. The intensity of neutrons from spontaneous fission and $(\alpha, n)$ reaction on oxygen executed with the data [2]. The intensity of gamma-emission of fuel estimated on the basis ABBN93 data from [3]. The group energy structure corresponds to the ABBN data set. Neutron spectra data via energy of $\alpha$-particles was compiled and used for the computation of neutron sources caused by $\alpha, n$ reaction on oxygen in dioxide fuel. The JAERI spectra were averaged into the ABBN 26 group energy range.

The dose rates and transport calculations were performed with ANISN code. The CONSYST code [4] with 26 neutron and 15 photon group ABBN-90 data set was used for calculations of mixture crosssections and transferring data to the CCC-254/ANISN formats. 
[1] Kochetkov A. " Code Care - calculations of isotopic kinetics, radioactive and ecological characterictics of nuclear fuel under raduation and cooling". Report IPPE 2431, 1995( in Russian)

[2] JAERI 1324, Data Book for Calculating Neutron Yields from $(\alpha, n)$ Reaction and Spontaneous Fission, 1992

[3] MKRZ: Decay scheme of radionuclides . Energy and Intensity of radiation. Moscow.Energoatomizdat, 1992.

[4] RSICC DLC-182 "ABBN-90: Multigroup Constant Set for Calculation of Neutron and Photon Radiation Fields and Functionals, Including the CONSYST2 Program".

\section{IPPE-Z (S. Zabrodskaia, G. Manturov)}

The sources of neutron and gamma emission were computed with ORIGEN-S code [1] from the American SCALE4.3 system. Under these calculations all needed for ORIGEN-S averaged cross-sections were calculated by the COCNSYST code [2] with ABBN-93 data set [3].

The dose rates and transport calculations were performed with TWODANT code in $\mathrm{P}_{3}$ order of anisotropy approximation using group constants set ABBN-93 with 299 neutron and 15 photon groups. The CONSYST code was used for calculations of mixture cross-sections and transferring data to the CCC547/TWODANT formats.

[1] O.W.Hermann, R.M.Westfall. ORIGEN-S: SCALE system module to calculate fuel depletion, actinide transmutation, fission product buildup and decay, and association source terms. SCALE4.3, Vol.2, Section F7, 1995

[2] RSICC DLC-182 "ABBN-90: Multigroup Constant Set for Calculation of Neutron and Photon Radiation Fields and Functionals, Including the CONSYST2 Program".

[3] G.N. Manturov, M.N. Nikolaev, A.M. Tsiboulia. ABBN-93 Group Data Library. Part 1. Nuclear Data for Calculation of Neutron and Photon Radiation Fields. Vienna, IAEA, INDC(CCP)-409/L, 1997, p.65-110.

\section{IPPE-L (V. Levanov)}

The radiation characteristics of actinides used at the calculations were taken from the publication 38 ICRP [1]. The yields of neutrons per decay took from Ref. [2].

About $50 \%$ of the neutron source is caused by (a-n) reaction on oxygen. It was assumed that the spectrum of neutrons is the fission spectrum.

Since the actinides in fresh fuel irradiate very soft gamma-radiation ( $\mathrm{Ag}<50 \mathrm{keV}$ ) which is not taken into consideration in the constants system CASK, the dose rates calculations near the fresh FA (without shielding) was carried out by means of analytical expressions. The shielding effect of an external wall outside fuel rods has been taken into consideration too.

The dose rates calculations near fresh FA and with the container were carried out with the code ANISN using the constants system CASK.

[1] MKRZ: Decay scheme of radionuclides. Energy and Intensity of radiation. Moscow.Energoatomizdat, 1992.

[2] JAERI 1324, part 2. Data book for calculations neutron yields from (a-n) reaction and spontaneous fission. 


\section{C.3 Comparison of the Calculation Results}

The participated results with their comparison presented in Tables C.1 to C.6. The Tables C.7 to C.21 present a comparison of the input data used by different participants.

Table C.1. UO2 - Comparison of dose rates $[\mu \mathrm{Sv} / \mathrm{h}]$ calculated by different methods

\begin{tabular}{|c|c|c|c|}
\hline $\begin{array}{c}\text { Comparison Case } \\
\text { UO2 }\end{array}$ & $\begin{array}{c}\text { [IPPE-K] } \\
\text { CARE+ } \\
\text { ANISN, } \\
\text { ABBN-90 } \\
26 \mathrm{~N}+15 \text { G }\end{array}$ & $\begin{array}{c}\text { [IPPE-Z] } \\
\text { ORIGEN+ } \\
\text { TWODANT, } \\
\text { ABBN-93 } \\
299 \text { N + 15 G }\end{array}$ & $\begin{array}{c}\text { [IPPE-L] } \\
\text { ANISN, } \\
\text { CASK } \\
22 \text { N + 18 G, } \\
\text { using analytic } \\
\text { expressions }\end{array}$ \\
\hline \multicolumn{4}{|l|}{ SOURCE: } \\
\hline neutron & 5727 & 5760 & 5790 \\
\hline gamma & $8.30 \mathrm{E}+9$ & $8.30 \mathrm{E}+9$ & $6.50 \mathrm{E}+9$ \\
\hline \multicolumn{4}{|l|}{ DOSE RATES: } \\
\hline \multicolumn{4}{|l|}{$\begin{array}{l}\text { Fuel Assembly } \\
\text { (FA) }\end{array}$} \\
\hline$k$-eff used & 0.08 & 0.08 & 0.10 \\
\hline neutron & 0.43 & 0.41 & 1.14 \\
\hline gamma & $20.8^{*}$ & $23.1 *$ & 28.0* \\
\hline On surface of FA & 21.2 & 23.6 & 29.1 \\
\hline At distance $0.5 \mathrm{~m}$ & 3.17 & 3.24 & 3.9 \\
\hline At distance $1.0 \mathrm{~m}$ & 1.75 & 1.74 & 2.0 \\
\hline At distance $2.0 \mathrm{~m}$ & 0.91 & 0.86 & 0.77 \\
\hline \multicolumn{4}{|l|}{ FA in a CASK } \\
\hline$k$-eff used & 0.33 & 0.33 & 0.32 \\
\hline neutron & 0.09 & 0.10 & 0.09 \\
\hline gamma & $3.28 *$ & $3.80^{*}$ & $2.70^{*}$ \\
\hline On surface of CASK & 3.37 & 3.90 & 2.80 \\
\hline At distance $0.5 \mathrm{~m}$ & 0.84 & 0.95 & 0.80 \\
\hline At distance $1.0 \mathrm{~m}$ & 0.46 & 0.53 & 0.47 \\
\hline At distance $2.0 \mathrm{~m}$ & 0.23 & 0.26 & 0.19 \\
\hline
\end{tabular}

* It was assumed that the U-238 is in equilibrium with Th-234 and Pa-234m 
Table C.2. MOX WEAPON - Comparison of dose rates $[\mu \mathrm{Sv} / \mathrm{h}]$ calculated by different methods

\begin{tabular}{|c|c|c|c|}
\hline $\begin{array}{c}\text { Comparison Case } \\
\text { MOX-W }\end{array}$ & $\begin{array}{c}\text { [IPPE-K] } \\
\text { CARE+ } \\
\text { ANISN, } \\
\text { ABBN-90 } \\
26 \text { N +15 G }\end{array}$ & $\begin{array}{c}\text { [IPPE-Z] } \\
\text { ORIGEN+ } \\
\text { TWODANT, } \\
\text { ABBN-93 } \\
299 \text { N + } 15 \text { G }\end{array}$ & $\begin{array}{c}\text { [IPPE-L] } \\
\text { ANISN, } \\
\text { CASK } \\
22 \text { N + } 18 \text { G, } \\
\text { using analytic } \\
\text { expressions } \\
\end{array}$ \\
\hline \multicolumn{4}{|l|}{ SOURCE } \\
\hline neutron & $2.04 \mathrm{E}+6$ & $2.13 \mathrm{E}+6$ & $1.92 \mathrm{E}+6$ \\
\hline gamma & $5.64 \mathrm{E}+12$ & $4.15 \mathrm{E}+12$ & $3.40 \mathrm{E}+12$ \\
\hline \multicolumn{4}{|l|}{ DOSE RATES: } \\
\hline \multicolumn{4}{|l|}{ Fuel Assembly (FA) } \\
\hline k-eff used & 0.10 & 0.10 & 0.10 \\
\hline neutron & 121 & 178 & 378 \\
\hline gamma & 133 & 24 & 184 \\
\hline On surface of EA & 254 & 202 & 562 \\
\hline At distance $0.5 \mathrm{~m}$ & 42 & 31 & 73.6 \\
\hline At distance $1.0 \mathrm{~m}$ & 23 & 18 & 35.2 \\
\hline At distance $2.0 \mathrm{~m}$ & 12 & 10 & 13.9 \\
\hline \multicolumn{4}{|l|}{ FA in a CASK } \\
\hline k-eff used & 0.34 & 0.34 & 0.32 \\
\hline neutron & 25.6 & 38.9 & 30.5 \\
\hline gamma & 18.8 & 4.4 & 26.0 \\
\hline On surface of CASK & 44.4 & 43.3 & 56.5 \\
\hline At distance $0.5 \mathrm{~m}$ & 12.9 & 12.7 & 16.0 \\
\hline At distance $1.0 \mathrm{~m}$ & 8.7 & 8.6 & 9.6 \\
\hline At distance $2.0 \mathrm{~m}$ & 5.9 & 6.1 & 3.8 \\
\hline
\end{tabular}


Table C.3. MOX REACTOR - Comparison of dose rates $[\mu \mathrm{Sv} / \mathrm{h}]$ calculated by different methods

\begin{tabular}{|c|c|c|c|}
\hline $\begin{array}{c}\text { Comparison Case } \\
\text { MOX-R }\end{array}$ & $\begin{array}{c}\text { [IPPE-K] } \\
\text { CARE+ } \\
\text { ANISN, } \\
\text { ABBN-90 } \\
26 \text { N +15 G }\end{array}$ & $\begin{array}{c}\text { [IPPE-Z] } \\
\text { ORIGEN+ } \\
\text { TWODANT, } \\
\text { ABBN-93 } \\
299 \text { N + } 15 \text { G }\end{array}$ & $\begin{array}{c}\text { [IPPE-L] } \\
\text { ANISN, } \\
\text { CASK } \\
22 \text { N + 18 G, } \\
\text { using } \\
\text { analytic } \\
\text { expressions }\end{array}$ \\
\hline \multicolumn{4}{|l|}{$\overline{\text { SOURCE }}$} \\
\hline neutron & $1.84 \mathrm{E}+7$ & $1.90 \mathrm{E}+7$ & $1.78 \mathrm{E}+7$ \\
\hline gamma & $8.23 \mathrm{E}+13$ & 10.3E+13 & $8.20 \mathrm{E}+13$ \\
\hline \multicolumn{4}{|l|}{ DOSE RATES: } \\
\hline \multicolumn{4}{|l|}{ Fuel Assembly (FA) } \\
\hline k-eff used & 0.11 & 0.11 & 0.10 \\
\hline neutron & 1290 & 1567 & 3500 \\
\hline gamma & 866 & 617 & 1970 \\
\hline On surface of EA & 2156 & 2184 & 5470 \\
\hline At distance $0.5 \mathrm{~m}$ & 345 & 338 & 793 \\
\hline At distance $1.0 \mathrm{~m}$ & 191 & 191 & 390 \\
\hline At distance $2.0 \mathrm{~m}$ & 102 & 105 & 159 \\
\hline \multicolumn{4}{|l|}{ FA in a CASK } \\
\hline$k$-eff used & 0.33 & 0.33 & 0.32 \\
\hline neutron & 240 & 335 & 282 \\
\hline gamma & 56 & 40 & 140 \\
\hline On surface of CASK & 296 & 375 & 422 \\
\hline At distance $0.5 \mathrm{~m}$ & 88 & 107 & 125 \\
\hline At distance $1.0 \mathrm{~m}$ & 61 & 71 & 73 \\
\hline At distance $2.0 \mathrm{~m}$ & 43 & 49 & 30 \\
\hline
\end{tabular}


Table C.4. Heat generation at fresh FA: $\mathrm{UO}_{2}(\mathrm{Wt})$

\begin{tabular}{|c|c|c|c|}
\hline Nuclide & IPPE-K & IPPE-Z & IPPE-L \\
\hline U-234 & $2.930 \mathrm{E}-02$ & $2.94 \mathrm{E}-02$ & $2.93 \mathrm{E}-02$ \\
\hline U-235 & $1.050 \mathrm{E}-03$ & $1.06 \mathrm{E}-03$ & $1.09 \mathrm{E}-03$ \\
\hline U-238 & $3.253 \mathrm{E}-03$ & $3.27 \mathrm{E}-03$ & $3.26 \mathrm{E}-03$ \\
\hline Total & $3.360 \mathrm{E}-02$ & $3.37 \mathrm{E}-02$ & $3.36 \mathrm{E}-02$ \\
\hline
\end{tabular}

Table C.5. Heat generation at fresh FA: MOX-W (4.2\%) (Wt)

\begin{tabular}{|c|c|c|c|}
\hline Nuclide & IPPE-K & IPPE-Z & IPPE-L \\
\hline U-234 & $7.032 \mathrm{E}-04$ & $7.03 \mathrm{E}-4$ & $7.03 \mathrm{E}-04$ \\
\hline $\mathrm{U}-235$ & $5.019 \mathrm{E}-05$ & $5.04 \mathrm{E}-5$ & $5.22 \mathrm{E}-05$ \\
\hline U-238 & $3.571 \mathrm{E}-03$ & $3.58 \mathrm{E}-03$ & $3.58 \mathrm{E}-03$ \\
\hline PU236 & $3.642 \mathrm{E}-04$ & $3.58 \mathrm{E}-04$ & \\
\hline Pu238 & $2.088 \mathrm{E}+00$ & $2.10 \mathrm{E}+00$ & $2.09 \mathrm{E}+00$ \\
\hline PU-239 & $3.331 \mathrm{E}+01$ & $3.33 \mathrm{E}+01$ & $3.34 \mathrm{E}+01$ \\
\hline PU-240 & $7.581 \mathrm{E}+00$ & $7.78 \mathrm{E}+00$ & $7.59 \mathrm{E}+00$ \\
\hline $\begin{array}{c}\text { PU-241 } \\
+\mathrm{U} 237\end{array}$ & $1.084 \mathrm{E}-01$ & $1.29 \mathrm{E}-01$ & $1.09 \mathrm{E}-01$ \\
\hline Pu242 & $6.398 \mathrm{E}-04$ & $6.52 \mathrm{E}-04$ & $6.41 \mathrm{E}-04$ \\
\hline Am-241 & $4.231 \mathrm{E}-01$ & $4.23 \mathrm{E}-01$ & $4.25 \mathrm{E}-01$ \\
\hline Total & $4.352 \mathrm{E}+01$ & $4.37 \mathrm{E}+01$ & $4.36 \mathrm{E}+01$ \\
\hline
\end{tabular}


Table C.6. Heat generation at fresh FA: MOX-R (6.1\%) (Wt)

\begin{tabular}{|c|c|c|c|}
\hline Nuclide & IPPE-K & IPPE-Z & IPPE-L \\
\hline U-234 & $7.032 \mathrm{E}-04$ & $7.06 \mathrm{E}-04$ & $7.03 \mathrm{E}-04$ \\
\hline U-235 & $4.925 \mathrm{E}-05$ & $4.95 \mathrm{E}-05$ & $1.06 \mathrm{E}-04$ \\
\hline U-238 & $3.503 \mathrm{E}-03$ & $3.53 \mathrm{E}-03$ & $3.51 \mathrm{E}-03$ \\
\hline Pu236 & $3.642 \mathrm{E}-02$ & $3.58 \mathrm{E}-02$ & \\
\hline Pu238 & $2.276 \mathrm{E}+02$ & $2.29 \mathrm{E}+02$ & $2.28 \mathrm{E}+02$ \\
\hline PU-239 & $2.979 \mathrm{E}+01$ & $2.99 \mathrm{E}+01$ & $2.99 \mathrm{E}+01$ \\
\hline PU-240 & $4.648 \mathrm{E}+01$ & $4.65 \mathrm{E}+01$ & $4.65 \mathrm{E}+01$ \\
\hline $\begin{array}{l}\text { PU-241 } \\
+\mathrm{U} 237\end{array}$ & $8.406 \mathrm{E}+00$ & $8.57 \mathrm{E}+00$ & $8.42 \mathrm{E}+00$ \\
\hline Pu242 & $1.643 \mathrm{E}-01$ & $1.69 \mathrm{E}-01$ & $1.65 \mathrm{E}-01$ \\
\hline Am-241 & $3.997 \mathrm{E}+01$ & $4.00 \mathrm{E}+01$ & $4.01 \mathrm{E}+01$ \\
\hline Total & $3.525 \mathrm{E}+02$ & $3.54 \mathrm{E}+02$ & $3.53 \mathrm{E}+02$ \\
\hline
\end{tabular}


Table C.7. Comparison of main radioactive characteristics: $T_{1 / 2}$ (year)

\begin{tabular}{|c|c|c|c|c|}
\hline Nuclide & $\begin{array}{c}\text { Type } \\
\text { of decay }\end{array}$ & IPPE-K & IPPE-Z & IPPE-L \\
\hline $\mathrm{U}-232$ & $\alpha$ & $7.200 \mathrm{E}+01$ & $6.9809 \mathrm{E}+01$ & $7.20 \mathrm{E}+01$ \\
\hline U-233 & $\alpha$ & $1.592 \mathrm{E}+05$ & $1.5920 \mathrm{E}+05$ & $1.59 \mathrm{E}+05$ \\
\hline U-234 & $\alpha$ & $2.446 \mathrm{E}+05$ & $2.4571 \mathrm{E}+05$ & $2.45 \mathrm{E}+05$ \\
\hline $\mathrm{U}-235$ & $\alpha$ & $7.040 \mathrm{E}+08$ & $7.0379 \mathrm{E}+08$ & $7.04 \mathrm{E}+08$ \\
\hline U-236 & $\alpha$ & $2.340 \mathrm{E}+07$ & $2.3421 \mathrm{E}+07$ & $2.34 \mathrm{E}+07$ \\
\hline U-237 & $\beta$ & $1.848 \mathrm{E}-02$ & & \\
\hline U-238 & $\alpha$ & $4.468 \mathrm{E}+09$ & $4.4680 \mathrm{E}+09$ & $4.47 \mathrm{E}+09$ \\
\hline Np237 (+Pa233) & $\alpha, \beta$ & $2.140 \mathrm{E}+06$ & $2.1399 \mathrm{E}+06$ & $2.14 \mathrm{E}+06$ \\
\hline $\mathrm{Np} 238$ & $\beta$ & 5.796E-03 & & \\
\hline Np239 & $\beta$ & 6.434E-03 & & \\
\hline $\mathrm{Pu} 236$ & $\alpha$ & $2.851 \mathrm{E}+00$ & & \\
\hline $\mathrm{Pu}-238$ & $\alpha$ & $8.774 \mathrm{E}+01$ & $8.7712 \mathrm{E}+01$ & $8.77 \mathrm{E}+01$ \\
\hline $\mathrm{Pu}-239$ & $\alpha$ & $2.410 \mathrm{E}+04$ & $2.4108 \mathrm{E}+04$ & $2.41 \mathrm{E}+04$ \\
\hline $\mathrm{Pu}-240$ & $\alpha$ & $6.560 \mathrm{E}+03$ & $6.5626 \mathrm{E}+03$ & $6.54 \mathrm{E}+03$ \\
\hline $\mathrm{Pu}-241$ (+U237) & $\beta, \alpha$ & $1.435 \mathrm{E}+01$ & $1.435 \mathrm{E}+01$ & $1.44 \mathrm{E}+01$ \\
\hline $\mathrm{Pu}-242$ & $\alpha$ & $3.763 \mathrm{E}+05$ & $3.7360 \mathrm{E}+05$ & $3.76 \mathrm{E}+05$ \\
\hline Am-241 & $\alpha$ & $4.320 \mathrm{E}+02$ & $4.3254 \mathrm{E}+02$ & $4.32 \mathrm{E}+02$ \\
\hline Am242 & $\beta, \mathrm{e}$ & 1.828E-03 & & \\
\hline Am242m ( +Am242) & $i, \alpha, \beta, \varepsilon$ & $1.520 \mathrm{E}+02$ & $1.4110 \mathrm{E}+02$ & $1.52 \mathrm{E}+02$ \\
\hline Am243 (+Np239) & $\alpha, \beta$ & $7.370 \mathrm{E}+03$ & $7.3706 \mathrm{E}+03$ & $7.38 \mathrm{E}+03$ \\
\hline $\mathrm{Cm}-242$ & $\alpha$ & 4.463E-01 & $4.4617 \mathrm{E}-01$ & $4.46 \mathrm{E}+01$ \\
\hline $\mathrm{Cm}-243$ & $\alpha, \varepsilon$ & $2.850 \mathrm{E}+01$ & $2.8557 \mathrm{E}+01$ & $2.85 \mathrm{E}+01$ \\
\hline $\mathrm{Cm}-244$ & $\alpha$ & $1.811 \mathrm{E}+01$ & $1.8100 \mathrm{E}+01$ & $1.81 \mathrm{E}+01$ \\
\hline $\mathrm{Cm}-245$ & $\alpha$ & $8.500 \mathrm{E}+03$ & $8.4987 \mathrm{E}+03$ & $8.50 \mathrm{E}+03$ \\
\hline $\mathrm{Cm}-246$ & $\alpha$ & $4.730 \mathrm{E}+03$ & & \\
\hline $\mathrm{Cm} 247$ & $\alpha$ & $1.560 \mathrm{E}+07$ & & \\
\hline $\mathrm{Cm} 248$ & $\alpha$ & $3.400 \mathrm{E}+05$ & & \\
\hline
\end{tabular}


Table C.8. Comparison of main radioactive characteristics :

Decay energy (MeV) and number of $\alpha$-particles per decay

\begin{tabular}{|c|c|c|c|c|c|c|c|}
\hline Nuclide & Type of & \multicolumn{2}{|c|}{ IPPE-K } & \multicolumn{2}{|c|}{ IPPE-Z } & \multicolumn{2}{|c|}{ IPPE-L } \\
\hline U-232 & $\alpha$ & 5.302 & 1 & 5.32 & 1 & 5.41 & 1 \\
\hline U-233 & $\alpha$ & 4.817 & 1 & 4.83 & 1 & 4.89 & 1 \\
\hline U-234 & $\alpha$ & 4.761 & 1 & 4.77 & 1 & 4.85 & 1 \\
\hline U-235 & $\alpha$ & 4.391 & 1 & 4.43 & 1 & 4.86 & 1 \\
\hline U-236 & $\alpha$ & 4.481 & 1 & 4.49 & & 4.59 & 1 \\
\hline U-237 & $\alpha$ & 0 & 0 & & 1 & & \\
\hline U-238 & $\alpha, \beta$ & 4.184 & 1 & 4.20 & 1 & 4.28 & 1 \\
\hline $\begin{array}{c}\mathrm{Np} 237 \\
(+\mathrm{Pa} 233) \\
\end{array}$ & $\alpha$ & 4.769 & 1 & 4.70 & & 5.34 & 1 \\
\hline Np238 & $\alpha$ & 0 & 0 & & & & \\
\hline Np239 & $\alpha$ & 0 & 0 & & & & \\
\hline Pu236 & $\beta, \alpha$ & 5.753 & 1 & & 1 & & \\
\hline $\mathrm{Pu}-238$ & $\alpha$ & 5.487 & 1 & 5.49 & 1 & 5.59 & 1 \\
\hline $\mathrm{Pu}-239$ & $\alpha$ & 5.148 & 1 & 5.15 & 1 & 5.24 & 1 \\
\hline $\mathrm{Pu}-240$ & $i, \alpha, \beta, \varepsilon$ & 5.156 & 1 & 5.16 & $2.39 \mathrm{E}-5$ & 5.25 & 1 \\
\hline $\begin{array}{c}\mathrm{Pu}-241 \\
(+\mathrm{U} 237)\end{array}$ & $\alpha, \varepsilon$ & 4.893 & $2.45 \mathrm{E}-5$ & 4.89 & 1 & $\begin{array}{c}0.0536+ \\
0.336\end{array}$ & $2.0 \mathrm{E}-5$ \\
\hline $\mathrm{Pu}-242$ & $\alpha$ & 4.891 & 1 & 4.90 & 1 & 4.98 & 1 \\
\hline Am-241 & $\alpha, \varepsilon$ & 5.479 & 1 & 5.48 & & 5.66 & 1 \\
\hline Am242 & $\alpha$ & 0 & 0 & & $4.5 \mathrm{E}-03$ & & \\
\hline $\begin{array}{c}\text { Am242m } \\
(+A m 242)\end{array}$ & $\alpha$ & 5.207 & 0.0045 & 5.22 & 1 & 0.271 & 4.8E-03 \\
\hline $\begin{array}{c}\text { Am243 } \\
(+\mathrm{Np} 239)\end{array}$ & & 5.271 & 1 & 5.28 & 1 & 5.87 & 1 \\
\hline $\mathrm{Cm}-242$ & & 6.102 & 1 & 6.04 & 1 & 6.22 & 1 \\
\hline Cm-243 & & 5.813 & 0.9976 & 5.85 & 1 & 6.15 & 1 \\
\hline Cm-244 & & 5.795 & 1 & 5.80 & 1 & 5.9 & 1 \\
\hline $\mathrm{Cm}-245$ & & 5.361 & 1 & 5.43 & & 5.61 & 1 \\
\hline Cm-246 & & 5.377 & 1 & & & & \\
\hline $\mathrm{Cm} 247$ & & 4.918 & 1 & & & & \\
\hline $\mathrm{Cm} 248$ & & 5.07 & 0.9174 & & & & \\
\hline
\end{tabular}


Table C.9. Comparison of main radioactive characteristics: yield of reaction $(\alpha-n)$ at $\mathrm{UO}_{2}$ (1/decay)

\begin{tabular}{|c|c|c|c|c|}
\hline Nuclide & $\begin{array}{l}\text { Type of } \\
\text { decay }\end{array}$ & IPPE-K & IPPE-Z & IPPE-L* \\
\hline U-232 & $\alpha$ & 1.90E-08 & $2.085 \mathrm{E}-08$ & $1.90 \mathrm{E}-08$ \\
\hline U-233 & $\alpha$ & 1.39E-08 & $1.4290 \mathrm{E}-08$ & $1.39 \mathrm{E}-08$ \\
\hline U-234 & $\alpha$ & 1.33E-08 & $1.3592 \mathrm{E}-08$ & $1.33 \mathrm{E}-08$ \\
\hline U-235 & $\alpha$ & 8.96E-09 & $9.9762 \mathrm{E}-09$ & $8.96 \mathrm{E}-09$ \\
\hline U-236 & $\alpha$ & $1.01 \mathrm{E}-08$ & $1.0595 \mathrm{E}-08$ & $1.01 \mathrm{E}-08$ \\
\hline U-237 & $\beta$ & $0.00 \mathrm{E}+00$ & & \\
\hline U-238 & $\alpha$ & 6.52E-09 & $7.7276 \mathrm{E}-09$ & $6.52 \mathrm{E}-09$ \\
\hline $\begin{array}{c}\mathrm{Np} 237 \\
(+\mathrm{Pa} 233)\end{array}$ & $\alpha, \beta$ & 1.34E-08 & $1.2868 \mathrm{E}-08$ & $1.34 \mathrm{E}-08$ \\
\hline $\mathrm{Np} 238$ & $\bar{\beta}$ & $0.00 \mathrm{E}+00$ & & \\
\hline Np239 & $\bar{\beta}$ & $0.00 \mathrm{E}+00$ & & \\
\hline $\mathrm{Pu} 236$ & $\alpha$ & $2.59 E-08$ & & \\
\hline $\mathrm{Pu}-238$ & $\alpha$ & $2.18 \mathrm{E}-08$ & $2.3443 \mathrm{E}-08$ & $2.18 \mathrm{E}-08$ \\
\hline $\mathrm{Pu}-239$ & $\alpha$ & $1.73 \mathrm{E}-08$ & $1.8403 \mathrm{E}-08$ & $1.73 \mathrm{E}-08$ \\
\hline $\mathrm{Pu}-240$ & $\alpha$ & 1.74E-08 & $1.8541 \mathrm{E}-08$ & $1.74 \mathrm{E}-08$ \\
\hline $\begin{array}{c}\mathrm{Pu}-241 \\
(+\mathrm{U} 237)\end{array}$ & $\beta, \alpha$ & 1.46E-08 & $1.5017 \mathrm{E}-08$ & \\
\hline $\mathrm{Pu}-242$ & $\alpha$ & 1.46E-08 & $1.5140 \mathrm{E}-08$ & $1.46 \mathrm{E}-08$ \\
\hline Am-241 & $\alpha$ & $2.17 \mathrm{E}-08$ & $2.3287 \mathrm{E}-08$ & $2.17 \mathrm{E}-08$ \\
\hline Am242 & $\beta, \mathrm{e}$ & $0.00 \mathrm{E}+00$ & & \\
\hline $\begin{array}{c}\text { Am242m } \\
(+\mathrm{Am} 242) \\
\end{array}$ & $\mathrm{i}, \alpha, \beta, \varepsilon$ & $1.79 \mathrm{E}-08$ & $1.9383 \mathrm{E}-08$ & $1.79 \mathrm{E}-08$ \\
\hline $\begin{array}{c}\text { Am243 } \\
(+\mathrm{Np} 239) \\
\end{array}$ & $\alpha, \beta$ & 1.86E-08 & $2.0272 \mathrm{E}-08$ & $1.86 \mathrm{E}-08$ \\
\hline $\mathrm{Cm}-242$ & $\alpha$ & 3.31E-08 & $3.2823 \mathrm{E}-08$ & $3.31 \mathrm{E}-08$ \\
\hline $\mathrm{Cm}-243$ & $\alpha, \varepsilon$ & $2.68 \mathrm{E}-08$ & $2.9445 \mathrm{E}-08$ & $2.68 \mathrm{E}-08$ \\
\hline $\mathrm{Cm}-244$ & $\alpha$ & $2.65 \mathrm{E}-08$ & $2.8580 \mathrm{E}-08$ & $2.65 \mathrm{E}-08$ \\
\hline $\mathrm{Cm}-245$ & $\alpha$ & 1.97E-08 & $2.2514 \mathrm{E}-08$ & $1.97 \mathrm{E}-08$ \\
\hline $\mathrm{Cm}-246$ & $\alpha$ & 1.99E-08 & & \\
\hline $\mathrm{Cm} 247$ & $\alpha$ & $1.51 \mathrm{E}-08$ & & \\
\hline $\mathrm{Cm} 248$ & $\alpha$ & 1.64E-08 & & \\
\hline
\end{tabular}

* Yield per any decay event 
Table C.10. Comparison of main radioactive characteristics: spontaneous fission (1/decay)

\begin{tabular}{|c|c|c|c|c|}
\hline Nuclide & \begin{tabular}{|c|} 
Type of \\
decay
\end{tabular} & IPPE-K & IPPE-Z & IPPE-L \\
\hline U-232 & $\alpha$ & $1.288 \mathrm{E}-12$ & $1.2819 \mathrm{E}-12$ & $2.80 \mathrm{E}-12$ \\
\hline U-233 & $\alpha$ & $1.948 \mathrm{E}-12$ & & $3.20 \mathrm{E}-12$ \\
\hline U-234 & $\alpha$ & $1.975 \mathrm{E}-11$ & $2.9237 \mathrm{E}-11$ & $3.20 \mathrm{E}-11$ \\
\hline U-235 & $\alpha$ & 3.409E-09 & $1.2201 \mathrm{E}-10$ & $4.80 \mathrm{E}-09$ \\
\hline U-236 & $\alpha$ & 1.931E-09 & $1.0000 \mathrm{E}-09$ & $1.40 \mathrm{E}-09$ \\
\hline U-237 & $\beta$ & 5.491E-22 & & \\
\hline U-238 & $\alpha$ & 1.091E-06 & $1.0900 \mathrm{E}-06$ & $1.10 \mathrm{E}-06$ \\
\hline $\mathrm{Np} 237(+\mathrm{Pa} 233)$ & $\alpha, \beta$ & $4.008 \mathrm{E}-12$ & & \\
\hline $\mathrm{Np} 238$ & $\beta$ & 5.689E-21 & & \\
\hline Np239 & $\beta$ & 2.097E-19 & & \\
\hline $\mathrm{Pu} 236$ & $\alpha$ & 1.808E-09 & & \\
\hline $\mathrm{Pu}-238$ & $\alpha$ & 4.194E-09 & $4.0000 \mathrm{E}-09$ & $4.20 \mathrm{E}-09$ \\
\hline $\mathrm{Pu}-239$ & $\alpha$ & $9.851 \mathrm{E}-12$ & $9.8560 \mathrm{E}-12$ & $1.20 \mathrm{E}-11$ \\
\hline $\mathrm{Pu}-240$ & $\alpha$ & 1.232E-07 & $1.2300 \mathrm{E}-07$ & $1.09 \mathrm{E}-07$ \\
\hline $\mathrm{Pu}-241$ (+U237) & $\beta, \alpha$ & $1.292 \mathrm{E}-14$ & & \\
\hline $\mathrm{Pu}-242$ & $\alpha$ & 1.183E-05 & $1.1803 \mathrm{E}-05$ & $1.23 \mathrm{E}-05$ \\
\hline Am-241 & $\alpha$ & $1.312 \mathrm{E}-11$ & $8.9820 \mathrm{E}-12$ & $1.20 \mathrm{E}-11$ \\
\hline Am242 & $\beta, \mathrm{e}$ & $4.524 \mathrm{E}-13$ & & \\
\hline $\begin{array}{c}\text { Am242m } \\
(+\mathrm{Am} 242)\end{array}$ & $i, \alpha, \beta, \varepsilon$ & $4.144 \mathrm{E}-10$ & $4.1440 \mathrm{E}-10$ & $5.10 \mathrm{E}-10$ \\
\hline Am243 (+Np239) & $\alpha, \beta$ & $5.544 \mathrm{E}-10$ & $9.3240 \mathrm{E}-11$ & $3.20 \mathrm{E}-10$ \\
\hline $\mathrm{Cm}-242$ & $\alpha$ & $1.601 \mathrm{E}-07$ & $1.6300 \mathrm{E}-07$ & $1.84 \mathrm{E}-07$ \\
\hline $\mathrm{Cm}-243$ & $\alpha, \varepsilon$ & $8.146 \mathrm{E}-10$ & & \\
\hline $\mathrm{Cm}-244$ & $\alpha$ & 3.649E-06 & $3.7170 \mathrm{E}-06$ & $3.73 \mathrm{E}-06$ \\
\hline $\mathrm{Cm}-245$ & $\alpha$ & 7.969E-09 & & \\
\hline $\mathrm{Cm}-246$ & $\alpha$ & 7.657E-04 & & \\
\hline $\mathrm{Cm} 247$ & $\alpha$ & $0.000 \mathrm{E}+00$ & & \\
\hline $\mathrm{Cm} 248$ & $\alpha$ & $2.583 \mathrm{E}-01$ & & \\
\hline
\end{tabular}


Table C.11. Masses of actinides at 1 FA of fresh fuel

$\mathrm{UO}_{2}(\mathrm{~g})$

\begin{tabular}{|c|c|c|c|}
\hline Nuclide & IPPE-K & IPPE-Z & IPPE-L \\
\hline $\mathrm{U}-234$ & $1.603 \mathrm{E}+02$ & $1.63 \mathrm{E}+02$ & $1.63 \mathrm{E}+02$ \\
\hline $\mathrm{U}-235$ & $1.758 \mathrm{E}+04$ & $1.76 \mathrm{E}+04$ & $1.76 \mathrm{E}+04$ \\
\hline $\mathrm{U}-238$ & $3.819 \mathrm{E}+05$ & $3.82 \mathrm{E}+05$ & $3.82 \mathrm{E}+05$ \\
\hline Total & $3.997 \mathrm{E}+05$ & $4.00 \mathrm{E}+5$ & $4.00 \mathrm{E}+5$ \\
\hline
\end{tabular}

Table C.12. Masses of actinides at $1 \mathrm{FA}$ of fresh fuel MOX-W (4.2\%) (g)

\begin{tabular}{|c|c|c|c|}
\hline Nuclide & IPPE-K & IPPE-Z & IPPE-L \\
\hline $\mathrm{U}-234$ & $4.006 \mathrm{E}+00$ & $3.92 \mathrm{E}+00$ & $3.91 \mathrm{E}+00$ \\
\hline $\mathrm{U}-235$ & $8.402 \mathrm{E}+02$ & $8.41 \mathrm{E}+02$ & $8.38 \mathrm{E}+02$ \\
\hline $\mathrm{U}-238$ & $4.192 \mathrm{E}+05$ & $4.20 \mathrm{E}+05$ & $4.19 \mathrm{E}+05$ \\
\hline $\mathrm{Pu}-236$ & $2.020 \mathrm{E}-05$ & & \\
\hline $\mathrm{Pu}-238$ & $3.686 \mathrm{E}+00$ & $3.69 \mathrm{E}+00$ & $3.69 \mathrm{E}+00$ \\
\hline $\mathrm{Pu}-239$ & $1.731 \mathrm{E}+04$ & $1.73 \mathrm{E}+04$ & $1.73 \mathrm{E}+04$ \\
\hline $\mathrm{Pu}-240$ & $1.071 \mathrm{E}+03$ & $1.07 \mathrm{E}+03$ & $1.07 \mathrm{E}+03$ \\
\hline $\begin{array}{l}\mathrm{Pu}-241 \\
+\mathrm{U} 237\end{array}$ & $3.317 \mathrm{E}+01$ & $3.32 \mathrm{E}+01$ & $3.32 \mathrm{E}+01$ \\
\hline $\mathrm{Pu}-242$ & $5.529 \mathrm{E}+00$ & $5.54 \mathrm{E}+00$ & $5.53 \mathrm{E}+00$ \\
\hline $\mathrm{Am}-241$ & $3.686 \mathrm{E}+00$ & $3.69 \mathrm{E}+00$ & $3.69 \mathrm{E}+00$ \\
\hline Total & $4.385 \mathrm{E}+05$ & $4.39 \mathrm{E}+05$ & $4.39 \mathrm{E}+05$ \\
\hline
\end{tabular}


Table C.13. Masses of actinides at $1 \mathrm{FA}$ of fresh fuel

MOX-R (6.1\%) (g)

\begin{tabular}{|c|c|c|c|}
\hline Nuclide & IPPE-K & IPPE-Z & IPPE-L \\
\hline $\mathrm{U}-234$ & $4.006 \mathrm{E}+00$ & $3.92 \mathrm{E}+00$ & $3.91 \mathrm{E}+00$ \\
\hline $\mathrm{U}-235$ & $8.241 \mathrm{E}+02$ & $8.25 \mathrm{E}+02$ & 825 \\
\hline $\mathrm{U}-238$ & $4.112 \mathrm{E}+05$ & $4.12 \mathrm{E}+05$ & $4.11 \mathrm{E}+05$ \\
\hline $\mathrm{Pu}-236$ & $2.020 \mathrm{E}-03$ & & \\
\hline $\mathrm{Pu}-238$ & $4.018 \mathrm{E}+02$ & $4.02 \mathrm{E}+02$ & $4.02 \mathrm{E}+02$ \\
\hline $\mathrm{Pu}-239$ & $1.548 \mathrm{E}+04$ & $1.55 \mathrm{E}+04$ & $1.55 \mathrm{E}+04$ \\
\hline $\mathrm{Pu}-240$ & $6.563 \mathrm{E}+03$ & $6.57 \mathrm{E}+03$ & $6.56 \mathrm{E}+03$ \\
\hline $\begin{array}{l}\mathrm{Pu}-241 \\
+\mathrm{U} 237\end{array}$ & $2.572 \mathrm{E}+03$ & $2.57 \mathrm{E}+03$ & $2.57 \mathrm{E}+03$ \\
\hline $\mathrm{Pu}-242$ & $1.420 \mathrm{E}+03$ & $1.42 \mathrm{E}+03$ & $1.42 \mathrm{E}+03$ \\
\hline Am-241 & $3.482 \mathrm{E}+02$ & $3.49 \mathrm{E}+02$ & $3.49 \mathrm{E}+02$ \\
\hline Total & $4.389 \mathrm{E}+05$ & $4.39 \mathrm{E}+05$ & $4.40 \mathrm{E}+05$ \\
\hline
\end{tabular}

Table C.14. Neutron source strength at 1 FA of fresh fuel $\mathrm{UO}_{2}(\mathrm{n} / \mathrm{s})$

\begin{tabular}{|c|c|c|c|}
\hline Nuclide & \multicolumn{1}{|c|}{ IPPE-K } & IPPE-Z & IPPE-L \\
\hline $\mathrm{U}-234$ & $4.965 \mathrm{E}+02$ & $5.11 \mathrm{E}+02$ & $5.03 \mathrm{E}+02$ \\
\hline $\mathrm{U}-235$ & $1.739 \mathrm{E}+01$ & $1.42 \mathrm{E}+01$ & $1.93 \mathrm{E}+01$ \\
\hline $\mathrm{U}-238$ & $5.218 \mathrm{E}+03$ & $5.23 \mathrm{E}+03$ & $5.26 \mathrm{E}+03$ \\
\hline Total & $5.727 \mathrm{E}+03$ & $5.76 \mathrm{E}+03$ & $5.79 \mathrm{E}+03$ \\
\hline
\end{tabular}


Table C.15. Neutron source strength at 1 FA of fresh fuel MOX-W (4.2\%) (n/s)

\begin{tabular}{|c|c|c|c|}
\hline Nuclide & IPPE-K & IPPE-Z & IPPE-L \\
\hline $\mathrm{U}-234$ & $1.241 \mathrm{E}+01$ & $1.23 \mathrm{E}+01$ & $1.21 \mathrm{E}+01$ \\
\hline $\mathrm{U}-235$ & $8.314 \mathrm{E}-01$ & $6.79 \mathrm{E}-01$ & $9.23 \mathrm{E}-01$ \\
\hline $\mathrm{U}-238$ & $5.728 \mathrm{E}+03$ & $5.73 \mathrm{E}+03$ & $5.78 \mathrm{E}+03$ \\
\hline $\mathrm{Pu}-236$ & $1.107 \mathrm{E}+01$ & $1.14 \mathrm{E}+01$ & \\
\hline $\mathrm{Pu}-238$ & $6.072 \mathrm{E}+04$ & $6.48 \mathrm{E}+04$ & $6.08 \mathrm{E}+04$ \\
\hline $\mathrm{Pu}-239$ & $6.886 \mathrm{E}+05$ & $7.29 \mathrm{E}+05$ & $6.90 \mathrm{E}+05$ \\
\hline $\mathrm{Pu}-240$ & $1.266 \mathrm{E}+06$ & $1.31 \mathrm{E}+06$ & $1.14 \mathrm{E}+06$ \\
\hline $\begin{array}{l}\mathrm{Pu}-241 \\
+\mathrm{U} 237\end{array}$ & $3.891 \mathrm{E}+01$ & $5.56 \mathrm{E}-01$ & \\
\hline $\mathrm{Pu}-242$ & $9.516 \mathrm{E}+03$ & $9.647 \mathrm{E}+03$ & $9.90 \mathrm{E}+03$ \\
\hline \begin{tabular}{c} 
Am-241 \\
\hline Total
\end{tabular} & $1.017 \mathrm{E}+04$ & $1.09 \mathrm{E}+04$ & $1.02 \mathrm{E}+04$ \\
\hline
\end{tabular}

Table C.16. Neutron source strength at $1 \mathrm{FA}$ of fresh fuel MOX-R (6.1\%) (n/s)

\begin{tabular}{|c|c|c|c|}
\hline Nuclide & IPPE-K & IPPE-Z & IPPE-L \\
\hline U-234 & $1.21 \mathrm{E}+01$ & $1.23 \mathrm{E}+01$ & $1.21 \mathrm{E}+01$ \\
\hline $\mathrm{U}-235$ & $1.87 \mathrm{E}+00$ & $6.60 \mathrm{E}-01$ & $1.87 \mathrm{E}+00$ \\
\hline U-238 & $5.67 \mathrm{E}+03$ & $5.62 \mathrm{E}+03$ & $5.67 \mathrm{E}+03$ \\
\hline Pu236 & & $1.14 \mathrm{E}+03$ & \\
\hline Pu238 & $6.63 \mathrm{E}+06$ & $7.07 \mathrm{E}+06$ & $6.63 \mathrm{E}+06$ \\
\hline PU-239 & $6.17 \mathrm{E}+05$ & $6.54 \mathrm{E}+05$ & $6.17 \mathrm{E}+05$ \\
\hline PU-240 & $7.00 \mathrm{E}+06$ & $7.86 \mathrm{E}+06$ & $7.00 \mathrm{E}+06$ \\
\hline $\begin{array}{c}\text { PU-241 } \\
+\mathrm{U} 237\end{array}$ & & $3.72 \mathrm{E}+03$ & \\
\hline Pu242 & $2.55 \mathrm{E}+06$ & $2.42 \mathrm{E}+06$ & $2.55 \mathrm{E}+06$ \\
\hline Am-241 & $9.62 \mathrm{E}+05$ & $1.03 \mathrm{E}+06$ & $9.62 \mathrm{E}+05$ \\
\hline Total & $1.842 \mathrm{E}+07$ & $1.90 \mathrm{E}+07$ & $1.78 \mathrm{E}+07$ \\
\hline
\end{tabular}


Table C.17. Neutron source spectrum normalized to 1 and total intensity at 1 FA calculated by IPPE-Z using ORIGEN (n/s)

\begin{tabular}{|c|c|c|c|c|}
\hline $\begin{array}{c}\mathbf{N} \\
\text { gr. }\end{array}$ & $\begin{array}{c}\text { Energy } \\
\text { boundaries, MeV }\end{array}$ & UO $_{2}$ & MOX-W & MOX-R \\
\hline 1 & $20.0-6.43$ & $1.611 \mathrm{E}-02$ & $1.077 \mathrm{E}-02$ & $1.268 \mathrm{E}-02$ \\
\hline 2 & $6.43-3.0$ & $2.072 \mathrm{E}-01$ & $2.365 \mathrm{E}-01$ & $2.277 \mathrm{E}-01$ \\
\hline 3 & $3.0-1.85$ & $2.744 \mathrm{E}-01$ & $3.572 \mathrm{E}-01$ & $3.285 \mathrm{E}-01$ \\
\hline 4 & $1.85-1.4$ & $1.329 \mathrm{E}-01$ & $1.254 \mathrm{E}-01$ & $1.271 \mathrm{E}-01$ \\
\hline 5 & $1.4-0.9$ & $1.646 \mathrm{E}-01$ & $1.277 \mathrm{E}-01$ & $1.397 \mathrm{E}-01$ \\
\hline 6 & $0.9-0.4$ & $1.715 \mathrm{E}-01$ & $1.192 \mathrm{E}-01$ & $1.374 \mathrm{E}-01$ \\
\hline 7 & $0.4-0.1$ & $3.350 \mathrm{E}-02$ & $2.322 \mathrm{E}-02$ & $2.687 \mathrm{E}-02$ \\
\hline $8-18$ & $0.1-0$ & 0 & 0 & 0 \\
\hline & Total & $5.76 \mathrm{E}+03$ & $2.13 \mathrm{E}+06$ & $1.90 \mathrm{E}+07$ \\
\hline
\end{tabular}

Table C.18. Gamma source spectrum normalized to 1 and total intensity at 1 FA calculated by IPPE-Z (photons/s)

\begin{tabular}{|c|c|c|c|c|}
\hline $\begin{array}{c}\mathbf{N} \\
\text { gr. }\end{array}$ & $\begin{array}{c}\text { Energy } \\
\text { boundaries, MeV }\end{array}$ & UO $_{2}$ & MOX-W & MOX-R \\
\hline $1-6$ & $11.0-2.5$ & 0 & 0 & 0 \\
\hline 7 & $2.5-1.75$ & $2.376 \mathrm{E}-04$ & $5.220 \mathrm{E}-07$ & $2.060 \mathrm{E}-08$ \\
\hline 8 & $1.75-1.25$ & $3.705 \mathrm{E}-04$ & $8.148 \mathrm{E}-07$ & $3.213 \mathrm{E}-08$ \\
\hline 9 & $1.25-0.75$ & $6.733 \mathrm{E}-03$ & $1.228 \mathrm{E}-05$ & $4.847 \mathrm{E}-07$ \\
\hline 10 & $0.75-0.35$ & $1.705 \mathrm{E}-03$ & $3.562 \mathrm{E}-06$ & $3.567 \mathrm{E}-06$ \\
\hline 11 & $0.35-0.15$ & $8.301 \mathrm{E}-02$ & $1.593 \mathrm{E}-04$ & $4.683 \mathrm{E}-04$ \\
\hline 12 & $0.15-0.08$ & $8.535 \mathrm{E}-02$ & $4.092 \mathrm{E}-04$ & $1.054 \mathrm{E}-03$ \\
\hline 13 & $0.08-0.04$ & $3.200 \mathrm{E}-02$ & $5.730 \mathrm{E}-02$ & $2.058 \mathrm{E}-01$ \\
\hline 14 & $0.04-0.02$ & $2.143 \mathrm{E}-02$ & $3.758 \mathrm{E}-03$ & $1.350 \mathrm{E}-02$ \\
\hline 15 & $0.02-0.01$ & $7.691 \mathrm{E}-01$ & $9.385 \mathrm{E}-01$ & $7.792 \mathrm{E}-01$ \\
\hline & Total & $8.305 \mathrm{E}+09$ & $4.149 \mathrm{E}+12$ & $1.030 \mathrm{E}+14$ \\
\hline
\end{tabular}


Table C.19. Neutron source spectrum normalized to 1 and total intensity at 1 FA calculated by IPPE-K using CARE (n/s)

\begin{tabular}{|c|c|c|c|c|}
\hline $\begin{array}{c}\text { N } \\
\text { gr. }\end{array}$ & $\begin{array}{c}\text { Energy } \\
\text { boundaries, MeV }\end{array}$ & MOX-W & MOX-R & UO $_{2}$ \\
\hline 1 & $14.5-6.5$ & $8.281 \mathrm{E}-03$ & $8.383 \mathrm{E}-03$ & $1.251 \mathrm{E}-02$ \\
\hline 2 & $6.5-4.5$ & $4.592 \mathrm{E}-02$ & $4.779 \mathrm{E}-02$ & $7.134 \mathrm{E}-02$ \\
\hline 3 & $4.0-2.5$ & $1.365 \mathrm{E}-01$ & $1.503 \mathrm{E}-01$ & $1.628 \mathrm{E}-01$ \\
\hline 4 & $2.5-1.4$ & $2.446 \mathrm{E}-01$ & $2.563 \mathrm{E}-01$ & $2.627 \mathrm{E}-01$ \\
\hline 5 & $1.4-0.8$ & $1.587 \mathrm{E}-01$ & $1.783 \mathrm{E}-01$ & $1.987 \mathrm{E}-01$ \\
\hline 6 & $0.8-0.4$ & $1.372 \mathrm{E}-01$ & $1.532 \mathrm{E}-01$ & $1.482 \mathrm{E}-01$ \\
\hline 7 & $0.4-0.2$ & $9.169 \mathrm{E}-02$ & $7.942 \mathrm{E}-02$ & $7.262 \mathrm{E}-02$ \\
\hline 8 & $0.2-0.1$ & $6.231 \mathrm{E}-02$ & $4.990 \mathrm{E}-02$ & $3.432 \mathrm{E}-02$ \\
\hline 9 & $0.1-0.0465$ & $6.279 \mathrm{E}-02$ & $4.214 \mathrm{E}-02$ & $2.182 \mathrm{E}-02$ \\
\hline 10 & $4.65 \mathrm{E}-2-2.15 \mathrm{E}-2$ & $2.837 \mathrm{E}-02$ & $1.877 \mathrm{E}-02$ & $8.810 \mathrm{E}-03$ \\
\hline 11 & $2.15 \mathrm{E}-2-1 . \mathrm{E}-2$ & $1.284 \mathrm{E}-02$ & $8.404 \mathrm{E}-03$ & $3.626 \mathrm{E}-03$ \\
\hline 12 & $1 \mathrm{E}-2-4.65 \mathrm{E}-3$ & $5.896 \mathrm{E}-03$ & $3.829 \mathrm{E}-03$ & $1.541 \mathrm{E}-03$ \\
\hline 13 & $4.65 \mathrm{E}-3-2.15 \mathrm{E}-3$ & $2.711 \mathrm{E}-03$ & $1.751 \mathrm{E}-03$ & $6.688 \mathrm{E}-04$ \\
\hline 14 & $2.15 \mathrm{E}-3-1 . \mathrm{E}-3$ & $1.243 \mathrm{E}-03$ & $7.998 \mathrm{E}-04$ & $2.942 \mathrm{E}-04$ \\
\hline 15 & $1 \mathrm{E}-3-4.65 \mathrm{E}-4$ & $5.767 \mathrm{E}-04$ & $3.699 \mathrm{E}-04$ & $1.323 \mathrm{E}-04$ \\
\hline 16 & $4.65 \mathrm{E}-4-2.15 \mathrm{E}-4$ & $2.670 \mathrm{E}-04$ & $1.709 \mathrm{E}-04$ & $5.995 \mathrm{E}-05$ \\
\hline 17 & $2.15 \mathrm{E}-4-1 . \mathrm{E}-4$ & $1.230 \mathrm{E}-04$ & $7.867 \mathrm{E}-05$ & $2.722 \mathrm{E}-05$ \\
\hline 18 & $1 \mathrm{E}-4-4.65 \mathrm{E}-5$ & $5.726 \mathrm{E}-05$ & $3.658 \mathrm{E}-05$ & $1.253 \mathrm{E}-05$ \\
\hline 19 & $4.65 \mathrm{E}-5-2.15 \mathrm{E}-5$ & $2.657 \mathrm{E}-05$ & $1.696 \mathrm{E}-05$ & $5.774 \mathrm{E}-06$ \\
\hline 20 & $2.15 \mathrm{E}-5-1 . \mathrm{E}-5$ & $1.226 \mathrm{E}-05$ & $7.826 \mathrm{E}-06$ & $2.652 \mathrm{E}-06$ \\
\hline 21 & $1 \mathrm{E}-5-4.65 \mathrm{E}-6$ & $5.713 \mathrm{E}-06$ & $3.645 \mathrm{E}-06$ & $1.231 \mathrm{E}-06$ \\
\hline 22 & $4.65 \mathrm{E}-6-2.15 \mathrm{E}-6$ & $2.653 \mathrm{E}-06$ & $1.692 \mathrm{E}-06$ & $5.705 \mathrm{E}-07$ \\
\hline 23 & $2.15 \mathrm{E}-6-1 . \mathrm{E}-6$ & $1.225 \mathrm{E}-06$ & $7.813 \mathrm{E}-07$ & $2.630 \mathrm{E}-07$ \\
\hline 24 & $1 \mathrm{E}-6-4.65 \mathrm{E}-7$ & $5.709 \mathrm{E}-07$ & $3.640 \mathrm{E}-07$ & $1.224 \mathrm{E}-07$ \\
\hline 25 & $4.65 \mathrm{E}-7-2.15 \mathrm{E}-7$ & $2.652 \mathrm{E}-07$ & $1.691 \mathrm{E}-07$ & $5.683 \mathrm{E}-08$ \\
\hline 26 & $2.53 \mathrm{E}-8$ & $2.289 \mathrm{E}-07$ & $1.460 \mathrm{E}-07$ & $4.903 \mathrm{E}-08$ \\
\hline Total & & $2.041 \mathrm{E}+06$ & $1.842 \mathrm{E}+07$ & $5.727 \mathrm{E}+03$ \\
\hline
\end{tabular}


Table C.20. Gamma source spectrum normalized to 1 and total intensity at 1 FA calculated by IPPE-K for storage time $t=0$ (photons/s)

\begin{tabular}{|c|c|c|c|c|}
\hline $\begin{array}{c}\mathbf{N} \\
\text { gr. }\end{array}$ & $\begin{array}{c}\text { Energy } \\
\text { boundaries, MeV }\end{array}$ & $\mathbf{U O}_{\mathbf{2}}$ & MOX-W & MOX-R \\
\hline $1-9$ & $11.0-0.75$ & & & \\
\hline 10 & $0.75-0.35$ & $9.643 \mathrm{E}-05$ & $6.837 \mathrm{E}-09$ & $3.050 \mathrm{E}-10$ \\
\hline 11 & $0.35-0.15$ & $1.172 \mathrm{E}-01$ & $8.314 \mathrm{E}-06$ & $3.709 \mathrm{E}-07$ \\
\hline 12 & $0.15-0.08$ & $5.910 \mathrm{E}-02$ & $4.191 \mathrm{E}-06$ & $1.869 \mathrm{E}-07$ \\
\hline 13 & $0.08-0.04$ & $1.083 \mathrm{E}-02$ & $4.227 \mathrm{E}-02$ & $1.816 \mathrm{E}-01$ \\
\hline 14 & $0.04-0.02$ & $0.000 \mathrm{E}+00$ & $2.775 \mathrm{E}-03$ & $1.192 \mathrm{E}-02$ \\
\hline 15 & $0.02-0.01$ & $8.128 \mathrm{E}-01$ & $9.549 \mathrm{E}-01$ & $8.065 \mathrm{E}-01$ \\
\hline & Total & $5.834 \mathrm{E}+09$ & $3.933 \mathrm{E}+12$ & $8.754 \mathrm{E}+13$ \\
\hline
\end{tabular}

Table C.21. Gamma source spectrum normalized to 1 and total intensity at 1 FA calculated by IPPE-K for storage time $t=10$ years (photons/s)

\begin{tabular}{|c|c|c|c|c|}
\hline $\begin{array}{c}\mathbf{N} \\
\text { gr. }\end{array}$ & $\begin{array}{c}\text { Energy } \\
\text { boundaries, }\end{array}$ & MOX-W & MOX-R & UO $_{2}$ \\
\hline 5 & $11-4.5$ & 0. & 0. & 0. \\
\hline 6 & $4.5-2.5$ & $6.348 \mathrm{E}-07$ & $1.643 \mathrm{E}-06$ & $0.000 \mathrm{E}+00$ \\
\hline 7 & $2.5-1.75$ & $3.945 \mathrm{E}-07$ & $1.945 \mathrm{E}-08$ & $2.422 \mathrm{E}-04$ \\
\hline 8 & $1.75-1.25$ & $7.205 \mathrm{E}-07$ & $2.118 \mathrm{E}-07$ & $3.994 \mathrm{E}-04$ \\
\hline 9 & $1.25-0.75$ & $9.642 \mathrm{E}-06$ & $6.531 \mathrm{E}-07$ & $5.870 \mathrm{E}-03$ \\
\hline 10 & $0.75-0.35$ & $3.430 \mathrm{E}-06$ & $3.490 \mathrm{E}-06$ & $1.335 \mathrm{E}-03$ \\
\hline 11 & $0.35-0.15$ & $3.882 \mathrm{E}-05$ & $6.560 \mathrm{E}-05$ & $8.318 \mathrm{E}-02$ \\
\hline 12 & $0.15-0.08$ & $1.221 \mathrm{E}-04$ & $1.406 \mathrm{E}-04$ & $8.577 \mathrm{E}-02$ \\
\hline 13 & $0.08-0.04$ & $1.303 \mathrm{E}-01$ & $2.741 \mathrm{E}-01$ & $3.220 \mathrm{E}-02$ \\
\hline 14 & $0.04-0.02$ & $8.553 \mathrm{E}-03$ & $1.799 \mathrm{E}-02$ & $2.145 \mathrm{E}-02$ \\
\hline 15 & $0.02-0.01$ & $8.609 \mathrm{E}-01$ & $7.077 \mathrm{E}-01$ & $7.696 \mathrm{E}-01$ \\
\hline & Total & $5.639 \mathrm{E}+12$ & $2.179 \mathrm{E}+14$ & $8.300 \mathrm{E}+09$ \\
\hline
\end{tabular}




\section{APPENDIX D}

CALCULATION RESULTS FOR TASK IIc: SHIELDING AND HEAT GENERATION STUDY FOR SPENT FUEL WITH A CASK 



\section{APPENDIX D}

\section{CALCULATION RESULTS FOR TASK IIc: SHIELDING AND HEAT GENERATION STUDY FOR SPENT FUEL WITH A CASK}

\section{D.1 The Desired Results}

In this task a study of shielding and radioactive characteristics of a Cask with spent fuel at a transportation is performed.

It is assumed a model of a Cask for 12 FAs with spent fuel. The geometric specifications of the Cask given in Table A.6 and in Fig.A.4 of the Benchmark Description.

Before moving FAs with spent fuel in the Cask it is assumed a storing its in a pool storage just like that which was described in Task I.

For calculation of the spent fuel compositions a pin-cell irradiation is to be performed with a discharge burnup of $60 \mathrm{GWd} / \mathrm{MTHM}$ at an average power $166 \mathrm{~W} / \mathrm{cm}$. The pin-cell cylinder specifications are: $\mathrm{r}_{1, \text { fuel }}=0.386 \mathrm{~cm}, \mathrm{r}_{2, \text { clad }}=0.4582 \mathrm{~cm}, \mathrm{r}_{3, \bmod }=0.7015 \mathrm{~cm}$. The initial fuel compositions are given in Table A. 2 of the Description. The composition of moderator is given in Table A.3. The operated temperatures should be used: $\mathrm{T}_{\text {fuel }}=1027 \mathrm{~K}, \mathrm{~T}_{\text {clad }}=579 \mathrm{~K}, \mathrm{~T}_{\bmod }=579 \mathrm{~K}$.

For each type of fuel should be calculated:

- Nuclide composition of actinides and fission products in spent fuel.

- Its activity.

- Dose rates at distance from the surface of the Cask equal to 0, 0.5, 1 and 2 meter.

- Heat generation for one FA total and fractionally for actinides and fission products in spent fuel via time of disposition 3 and 10 days, and 1, 3, 10 and 100 years.

\section{D.2 Short Description of the Used Methods}

Participated results:

\begin{tabular}{|l|l|}
\hline Participants & ID \\
\hline ABBN+WIMS/D4 +CARE + ANISN + ABBN-93 & IPPE-K \\
\hline \hline ABBN+MAYAK+ ORIGEN + TWODANT + ABBN-93 & IPPE-Z \\
\hline ABBN+MAYAK+ORIGEN+ ANISN + CASK & IPPE-L \\
\hline
\end{tabular}

IPPE-K (A. Kotchetkov, G. Khohlov, G.Jerdev)

The spent fuel composition was calculated using ABBN-WIMS system using WIMS/D4 for neutronics calculations

The sources of neutron and gamma emission for the spent fuel composition but also the heat generation were computed with the code CARE. 
The dose rates and transport calculations were performed with ANISN code. The CONSYST code with 26 neutron and 15 photon group ABBN-90 data set was used for calculations of mixture crosssections and transferring data to the CCC-254/ANISN formats.

\section{IPPE-Z (S. Zabrodskaia , G. Manturov, A.Tsiboulia)}

Code system MAYAK allows to calculate neutron-physical characteristics of reactor system with account of changes of their isotopic composition during burnup process. In this particular case, the KENOVI Monte-Carlo code was used for the neutronics calculations and the code ORIGEN-S used for calculation of the spent fuel composition taking into account the burnup of $60 \mathrm{GWd} / \mathrm{MTHM}$ at the average power $166 \mathrm{~W} / \mathrm{cm}$. The CONSYST code was used for 299 group mixture cross-section calculations in $\mathrm{P}_{3}$ order of anisotropy approximation with group constants set ABBN-93.

Under the ORIGEN calculations all original ORIGEN libraries of neutron cross sections are replaced by (1) the calculated with the CONSYST code and (2) data for all other nuclides are taken from external ABBN libraries of fission products FP and actinides ACT. The library of masses is used too.

The sources of neutron and gamma emission and the heat generation for the spent fuel composition were computed with ORIGEN-S code. Under these calculations all needed for ORIGEN averaged crosssections were calculated by the COCNSYST code with the ABBN-93 data set.

The dose rates and transport calculations were performed with TWODANT code in $\mathrm{P}_{3}$ order of anisotropy approximation using group constants set ABBN-93 with 299 neutron and 15 photon groups. The CONSYST code was used for calculations of mixture cross-sections and transferring data to the CCC547/TWODANT formats. Two types of calculations were performed: (1) 1-D calculations with infinite height of the Cask and (2) 2-D RZ calculations taking into account the real geometry of the Cask model.

\section{IPPE-L (V. Levanov, A.Tsiboulia)}

At the calculations of the radiation heating the actinides and fission products were taken into account. The heating from fission products calculated by formula:

$$
Q=N \cdot\left(q\left(t_{s}, a, k\right)-q\left(t_{o p}+t_{s}, a, k\right)\right)[\mathrm{W}]
$$

Function $q(t, a, k)$ :

$$
q(t, a, k)=\sum_{i=1}^{33} m_{i}(a, k) \cdot \exp \left(-n_{i} \cdot t\right)
$$

$N$-operating power, $\mathrm{W}$

$t_{s}$ - cooling time, $\mathrm{s}$

$t_{o p}$ - operating time, $\mathrm{s}$

$\mathrm{a}$ - contribution of $\mathrm{Pu}$ into fission power

$\mathrm{k}$ - factor for Cs -134

The dose rates calculations near the container with displaced 12 irradiated FAs were carried out with the code ANISN using the constants system CASK. 


\section{D.3 Comparison of the Calculation Results}

The participated results on the dose rates calculations and their comparison are given in Tables D.1D.7.

Table D.1. $\mathrm{UO}_{2}$ - Comparison of dose rates $[\mu \mathrm{Sv} / \mathrm{h}]$ calculated by different methods

\begin{tabular}{|c|c|c|c|c|}
\hline \multirow[t]{2}{*}{$\begin{array}{c}\text { Comparison Case } \\
\text { UO2 }\end{array}$} & $\begin{array}{c}\text { [IPPE-K] } \\
\text { CARE+ } \\
\text { ANISN, } \\
\text { ABBN-90 } \\
26 \text { N +15 G }\end{array}$ & \multicolumn{2}{|c|}{$\begin{array}{c}\text { [IPPE-M] } \\
\text { ORIGEN+ } \\
\text { TWODANT, } \\
\text { ABBN-93 } \\
299 \text { N + } 15 \text { G }\end{array}$} & \multirow[t]{2}{*}{$\begin{array}{c}\text { [IPPE-L] } \\
\text { ANISN, } \\
\text { CASK } \\
22 \mathrm{~N}+18 \mathrm{G}, \\
\text { using analytic } \\
\text { expressions } \\
\end{array}$} \\
\hline & & 2-D & 1-D & \\
\hline \multicolumn{5}{|l|}{ SOURCE: } \\
\hline neutron & $6.79 \mathrm{E}+8$ & $5.47 \mathrm{E}+8$ & $5.47 \mathrm{E}+8$ & $533 \mathrm{E}+8$ \\
\hline gamma & $7.71000 \mathrm{e}+15$ & $5.57 \mathrm{E}+15$ & $5.57 \mathrm{E}+15$ & \\
\hline \multicolumn{5}{|l|}{ DOSE RATES: } \\
\hline \multicolumn{5}{|l|}{12 FA in a CASK } \\
\hline$k$-eff used & 0.18 & 0.18 & 0.18 & 0.3 \\
\hline neutron & 59 & 60 & 62 & 170 \\
\hline gamma & 490 & 413 & 428 & 460 \\
\hline On surface of CASK & 550 & 473 & 490 & 630 \\
\hline At distance $0.5 \mathrm{~m}$ & 310 & 250 & 278 & 460 \\
\hline At distance $1.0 \mathrm{~m}$ & 234 & 174 & 210 & 350 \\
\hline At distance $2.0 \mathrm{~m}$ & 155 & 91 & 139 & 230 \\
\hline
\end{tabular}


Table D.2. MOX WEAPON - Comparison of dose rates $[\mu \mathrm{Sv} / \mathrm{h}]$ calculated by different methods

\begin{tabular}{|c|c|c|c|c|}
\hline \multirow[t]{2}{*}{$\begin{array}{c}\text { Comparison Case } \\
\text { MOX-R }\end{array}$} & \multirow[t]{2}{*}{$\begin{array}{c}\text { [IPPE-K] } \\
\text { CARE+ } \\
\text { ANISN, } \\
\text { ABBN-90 } \\
26 \text { N +15 G }\end{array}$} & \multicolumn{2}{|c|}{$\begin{array}{c}\text { [IPPE-M] } \\
\text { ORIGEN+ } \\
\text { TWODANT, } \\
\text { ABBN-93 } \\
299 \text { N + 15 G }\end{array}$} & \multirow[t]{2}{*}{$\begin{array}{c}\text { [IPPE-L] } \\
\\
\text { ANISN, } \\
\text { CASK } \\
22 \mathrm{~N}+18 \mathrm{G}, \\
\text { using analytic } \\
\text { expressions }\end{array}$} \\
\hline & & 2-D & 1-D & \\
\hline \multicolumn{5}{|l|}{ SOURCE } \\
\hline neutron & $1.89 \mathrm{E}+9$ & $1.67 \mathrm{E}+9$ & $1.65 \mathrm{E}+9$ & $1.62 \mathrm{E}+9$ \\
\hline gamma & $1.06 \mathrm{E}+16$ & $6.91+15$ & $6.91+15$ & \\
\hline \multicolumn{5}{|l|}{ DOSE RATES: } \\
\hline \multicolumn{5}{|l|}{ 12FA in a CASK } \\
\hline k-eff used & 0.21 & 0.21 & 0.21 & 0.30 \\
\hline neutron & 175 & 202 & 177 & 500 \\
\hline gamma & 875 & 973 & 1055 & 1060 \\
\hline On surface of CASK & 1050 & 1175 & 1233 & 1560 \\
\hline At distance $0.5 \mathrm{~m}$ & 2587 & 600 & 6888 & 1200 \\
\hline At distance $1.0 \mathrm{~m}$ & 4441 & 410 & 517 & 880 \\
\hline At distance $2.0 \mathrm{~m}$ & 291 & 208 & 342 & 570 \\
\hline
\end{tabular}


Table D.3. MOX REACTOR - Comparison of dose rates $[\mu \mathrm{Sv} / \mathrm{h}]$ calculated by different methods

\begin{tabular}{|c|c|c|c|c|}
\hline \multirow[t]{2}{*}{$\begin{array}{c}\text { Comparison Case } \\
\text { MOX-W }\end{array}$} & \multirow[t]{2}{*}{$\begin{array}{c}\text { [IPPE-K] } \\
\text { CARE+ } \\
\text { ANISN, } \\
\text { ABBN-90 } \\
28 \text { N +15 G }\end{array}$} & \multicolumn{2}{|c|}{$\begin{array}{c}\text { [IPPE-M] } \\
\text { ORIGEN+ } \\
\text { TWODANT, } \\
\text { ABBN-93 } \\
299 \text { N + 15 G }\end{array}$} & \multirow[t]{2}{*}{$\begin{array}{c}\text { [IPPE-L] } \\
\text { ANISN, } \\
\text { CASK } \\
22 \mathrm{~N}+18 \mathrm{G}, \\
\text { using analytic } \\
\text { expressions } \\
\end{array}$} \\
\hline & & 2-D & 1-D & \\
\hline \multicolumn{5}{|l|}{ SOURCE } \\
\hline neutron & $5.54 \mathrm{E}+9$ & $5.68 \mathrm{E}+9$ & $5.68 \mathrm{E}+9$ & $5.48 \mathrm{E}+9$ \\
\hline gamma & $1.03 \mathrm{E}+16$ & $6.75+15$ & $6.75+15$ & \\
\hline \multicolumn{5}{|l|}{ DOSE RATES: } \\
\hline \multicolumn{5}{|l|}{12 FA in a CASK } \\
\hline$k$-eff used & 0.26 & 0.26 & 0.26 & 0.30 \\
\hline neutron & 566 & 690 & 643 & 1700 \\
\hline gamma & 2100 & 2810 & 2821 & 3160 \\
\hline On surface of CASK & 2666 & 3500 & 3464 & 4860 \\
\hline At distance $0.5 \mathrm{~m}$ & 1473 & 1752 & 1906 & 3600 \\
\hline At distance $1.0 \mathrm{~m}$ & 1104 & 1173 & 1430 & 2700 \\
\hline At distance $2.0 \mathrm{~m}$ & 729 & 587 & 944 & 1800 \\
\hline
\end{tabular}


Table D.4. The masses of actinides in one irradiated FA(g) after 3 years cooling time UOX

\begin{tabular}{|c|c|c|}
\hline Nuclide & IPPE-K & IPPE-Z,-L \\
\hline U-232 *E-2 & 0.122 & 1.075 \\
\hline U-233 *E-3 & 0.872 & 3.605 \\
\hline$\overline{\mathrm{U}-234{ }^{*} \mathrm{E}+1}$ & 7.584 & 7.376 \\
\hline U-235 *E+3 & 2.181 & 2.549 \\
\hline $\bar{U}-236{ }^{\star} \mathrm{E}+3$ & 2.410 & 2.317 \\
\hline $\bar{U}$ U-237 *E-5 & & 2.108 \\
\hline U-238 *E+5 & 3.645 & 3.653 \\
\hline $\mathrm{Np}-237^{*} \mathrm{E}+2$ & 3.149 & 3.065 \\
\hline Np-238*E-8 & & 7.481 \\
\hline Np-239*E-5 & & 9.914 \\
\hline Pu-236*E-4 & 8.476 & 75.910 \\
\hline Pu-238*E+2 & 2.867 & 1.383 \\
\hline Pu-239*E+3 & 2.500 & 2.579 \\
\hline Pu-240*E+3 & 1.176 & 1.284 \\
\hline Pu-241*E+2 & 6.569 & 6.943 \\
\hline Pu-242*E+2 & 4.589 & 4.429 \\
\hline Am-241*E+2 & 1.237 & 1.317 \\
\hline Am-242*E-6 & 5.028 & 4.809 \\
\hline Am-242mE-1 & 4.181 & 3.726 \\
\hline $\mathrm{Am}^{2443^{*} \mathrm{E}+2}$ & 1.360 & 1.149 \\
\hline Clm-242*E-1 & 1.092 & 1.142 \\
\hline Cm-243*E-1 & 3.216 & 3.170 \\
\hline Cm-244*E+1 & 6.073 & 4.737 \\
\hline $\mathrm{Cm}-245^{\star} \mathrm{E}+0$ & 4.929 & 2.975 \\
\hline Cm-246*E-1 & 1.372 & 5.745 \\
\hline Cm-247*E-3 & 3.582 & 9.459 \\
\hline Cm-248*E-4 & 5.841 & 8.118 \\
\hline Total & $3.749 \mathrm{E}+05$ & $3.760 \mathrm{E}+05$ \\
\hline
\end{tabular}


Table D.5. The masses of actinides in one irradiated FA ( $\mathrm{g}$ ) after 3 years cooling time MOX (weapon)

\begin{tabular}{|c|c|c|}
\hline Nuclide & IPPE-K & IPPE-Z,-L \\
\hline $\bar{U}-232{ }^{*} \mathrm{E}-3$ & 0.428 & 3.750 \\
\hline U-233 *E-4 & 0.488 & 3.835 \\
\hline $\mathrm{U}-234{ }^{*} \mathrm{E}+0$ & 5.831 & 5.982 \\
\hline $\bar{U}-235^{*} \mathrm{E}+2$ & 2.040 & 2.126 \\
\hline $\mathrm{U}-236{ }^{*} \mathrm{E}+2$ & 1.110 & 1.105 \\
\hline $\bar{U}-237^{*} \mathrm{E}-5$ & & 5.724 \\
\hline $\bar{U}-238{ }^{*} \mathrm{E}+5$ & 3.998 & 3.993 \\
\hline Np-237E+1 & 7.909 & 7.726 \\
\hline Np-238*E-7 & & 3.771 \\
\hline Np-239*E-4 & & 2.586 \\
\hline Pu-236*E-3 & 0.272 & 2.462 \\
\hline Pu-238*E+2 & 1.459 & 1.506 \\
\hline Pu-239E+3 & 4.491 & 4.743 \\
\hline Pu-240*E+3 & 3.224 & 3.479 \\
\hline Pu-241*E+3 & 1.816 & 1.886 \\
\hline Pu-242*E+3 & 1.019 & 1.061 \\
\hline Am-241*E+2 & 3.821 & 3.995 \\
\hline Am-242*E-5 & 2.758 & 2.580 \\
\hline Am-242m*E+0 & 2.294 & 1.995 \\
\hline Am243*E+2 & 3.454 & 2.971 \\
\hline Clm-242*E-1 & 3.758 & 4.107 \\
\hline Cm-243*E+0 & 1.320 & 1.430 \\
\hline Cm-244*E+2 & 1.702 & 1.442 \\
\hline $\mathrm{Cm}-245^{\star} \mathrm{E}+1$ & 1.909 & 1.347 \\
\hline Cm-246*E+0 & 0.647 & 2.118 \\
\hline C Cm-247*E-2 & $\begin{array}{l}1.941 \\
\end{array}$ & 4.402 \\
\hline Cm-248*E-3 & 3.839 & 4.006 \\
\hline Total & $4.118 \mathrm{E}+05$ & $4.119 \mathrm{E}+05$ \\
\hline
\end{tabular}


Table D.6. The masses of actinides in one irradiated FA (g) after 3 years cooling time MOX (reactor)

\begin{tabular}{|c|c|c|}
\hline Nuclide & IPPE-K & IPPE-Z,-L \\
\hline U-232 *E-3 & 0.995 & 4.338 \\
\hline U-233 *E-4 & 2.252 & 7.599 \\
\hline$\overline{\mathrm{U}-234{ }^{*} \mathrm{E}+1}$ & 2.466 & 2.517 \\
\hline $\bar{U}-235{ }^{*} \mathrm{E}+2$ & 2.672 & 2.771 \\
\hline $\bar{U}-236{ }^{\star} \mathrm{E}+2$ & 1.054 & 1.062 \\
\hline $\bar{U}$ U-237 *E-5 & & 8.988 \\
\hline U-238 *E+5 & 3.924 & 3.920 \\
\hline Np-237*E+1 & 8.404 & 7.640 \\
\hline Np-238*E-6 & & 1.052 \\
\hline Np-239*E-4 & & 6.265 \\
\hline Pu-236*E-3 & 0.4239 & 2.594 \\
\hline Pu-238*E+2 & 5.891 & 6.027 \\
\hline Pu-239*E+3 & 6.108 & 6.478 \\
\hline Pu-240*E+3 & 4.931 & 5.265 \\
\hline Pu-241*E+3 & 2.849 & 2.963 \\
\hline Pu-242*E+3 & 2.426 & 2.350 \\
\hline Am-241*E+2 & 6.881 & 7.183 \\
\hline Am-242*E-5 & 7.964 & 7.385 \\
\hline Am-242m*E+0 & 6.624 & 5.718 \\
\hline Am243*E+2 & 7.171 & 7.248 \\
\hline Clm-242*E-1 & 7.977 & 8.689 \\
\hline Cm-243*E+0 & 3.853 & 4.244 \\
\hline $\mathrm{Cm}-244^{*} \mathrm{E}+2$ & 4.966 & 4.902 \\
\hline $\mathrm{Cm}-245^{\star} \mathrm{E}+1$ & 8.023 & 6.656 \\
\hline $\mathrm{Cm}-246^{*} \mathrm{E}+1$ & 3.846 & 1.074 \\
\hline Clm-247*E-1 & $\begin{array}{l}1.403 \\
\end{array}$ & 2.837 \\
\hline Cm-248*E-2 & 3.578 & 2.857 \\
\hline Total & $4.118 \mathrm{E}+05$ & $4.122 \mathrm{E}+05$ \\
\hline
\end{tabular}


Table D.7. Heat generation via cooling time for 1 irradiated FA, Wt

\begin{tabular}{|c|c|c|c|c|c|c|c|c|}
\hline Fuel & & $\begin{array}{l}\text { IPPE } \\
\text { results }\end{array}$ & 3 day & 10 day & 1 year & 3 year & 10 year & 100 year \\
\hline \multirow[t]{9}{*}{ MOX-W } & \multirow[t]{3}{*}{ Actin. } & $\mathbf{K}$ & $1.73 \mathrm{E}+4$ & $5.84 \mathrm{E}+3$ & $1.62 \mathrm{E}+3$ & $6.50 \mathrm{E}+2$ & $5.60 \mathrm{E}+2$ & $2.93 \mathrm{E}+2$ \\
\hline & & $\mathbf{Z}$ & $1.78 \mathrm{E}+4$ & $7.11 \mathrm{E}+3$ & $1.69 \mathrm{E}+3$ & $6.34 \mathrm{E}+2$ & $5.44 \mathrm{E}+2$ & $3.16 \mathrm{E}+2$ \\
\hline & & $\mathbf{L}$ & $5.75 \mathrm{E}+3$ & $5.60 \mathrm{E}+3$ & $1.68 \mathrm{E}+3$ & $6.14 \mathrm{E}+2$ & $5.18 \mathrm{E}+2$ & $2.82 \mathrm{E}+2$ \\
\hline & \multirow[t]{3}{*}{ FP } & $\mathbf{K}$ & $5.81 \mathrm{E}+4$ & $4.16 \mathrm{E}+4$ & $7.12 \mathrm{E}+3$ & $2.36 \mathrm{E}+3$ & $5.44 \mathrm{E}+2$ & $5.31 \mathrm{E}+1$ \\
\hline & & $\mathbf{Z}$ & $6.50 \mathrm{E}+4$ & $4.56 \mathrm{E}+4$ & $8.46 \mathrm{E}+3$ & $2.91 \mathrm{E}+3$ & $6.40 \mathrm{E}+2$ & $5.78 \mathrm{E}+1$ \\
\hline & & $\mathbf{L}$ & $6.62 \mathrm{E}+4$ & $4.54 \mathrm{E}+4$ & $7.38 \mathrm{E}+3$ & $2.46 \mathrm{E}+3$ & $6.38 \mathrm{E}+2$ & $6.59 \mathrm{E}+1$ \\
\hline & \multirow{3}{*}{ SUM } & $\mathbf{K}$ & $7.54 \mathrm{E}+4$ & $4.74 \mathrm{E}+4$ & $8.74 \mathrm{E}+3$ & $3.01 \mathrm{E}+3$ & $1.10 \mathrm{E}+3$ & $3.64 \mathrm{E}+2$ \\
\hline & & $\mathbf{Z}$ & $8.28 \mathrm{E}+4$ & $5.27 \mathrm{E}+4$ & $1.01 \mathrm{E}+4$ & $3.55 \mathrm{E}+3$ & $1.18 \mathrm{E}+3$ & $3.74 \mathrm{E}+2$ \\
\hline & & $\mathbf{L}$ & $7.19 \mathrm{E}+4$ & $5.10 \mathrm{E}+4$ & $9.08 \mathrm{E}+3$ & $3.08 \mathrm{E}+3$ & $1.16 \mathrm{E}+3$ & $3.48 \mathrm{E}+2$ \\
\hline \multirow[t]{9}{*}{ MOX-R } & \multirow[t]{3}{*}{ Actin. } & $\mathbf{K}$ & $2.27 \mathrm{E}+4$ & $1.21 \mathrm{E}+4$ & $3.98 \mathrm{E}+3$ & $1.86 \mathrm{E}+3$ & $1.50 \mathrm{E}+3$ & $5.65 \mathrm{E}+2$ \\
\hline & & $\mathbf{Z}$ & $2.37 \mathrm{E}+4$ & $1.38 \mathrm{E}+4$ & $4.26 \mathrm{E}+3$ & $1.99 \mathrm{E}+3$ & $1.64 \mathrm{E}+3$ & $6.22 \mathrm{E}+2$ \\
\hline & & $\mathbf{L}$ & $1.28 \mathrm{E}+4$ & $1.26 \mathrm{E}+4$ & $4.24 \mathrm{E}+3$ & $1.94 \mathrm{E}+3$ & $1.58 \mathrm{E}+3$ & $5.82 \mathrm{E}+2$ \\
\hline & \multirow[t]{3}{*}{$\mathbf{F P}$} & $\mathbf{K}$ & $5.81 \mathrm{E}+4$ & $4.19 \mathrm{E}+4$ & $7.19 \mathrm{E}+3$ & $2.35 \mathrm{E}+3$ & $5.35 \mathrm{E}+2$ & $5.29 \mathrm{E}+1$ \\
\hline & & $\mathbf{Z}$ & $6.39 \mathrm{E}+4$ & $4.45 \mathrm{E}+4$ & $8.41 \mathrm{E}+3$ & $2.88 \mathrm{E}+3$ & $6.34 \mathrm{E}+2$ & $5.72 \mathrm{E}+1$ \\
\hline & & $\mathbf{L}$ & $6.62 \mathrm{E}+4$ & $4.54 \mathrm{E}+4$ & $7.38 \mathrm{E}+3$ & $2.46 \mathrm{E}+3$ & $6.38 \mathrm{E}+2$ & $6.59 \mathrm{E}+1$ \\
\hline & \multirow[t]{3}{*}{ SUM } & $\mathbf{K}$ & $8.08 \mathrm{E}+4$ & $5.40 \mathrm{E}+4$ & $1.12 \mathrm{E}+4$ & $4.21 \mathrm{E}+3$ & $2.03 \mathrm{E}+3$ & $6.18 \mathrm{E}+2$ \\
\hline & & $\mathbf{Z}$ & $8.76 \mathrm{E}+4$ & $5.88 \mathrm{E}+4$ & $1.27 \mathrm{E}+4$ & $4.87 \mathrm{E}+3$ & $2.27 \mathrm{E}+3$ & $6.79 \mathrm{E}+2$ \\
\hline & & $\mathbf{L}$ & $7.90 \mathrm{E}+4$ & $5.79 \mathrm{E}+4$ & $1.16 \mathrm{E}+4$ & $4.40 \mathrm{E}+3$ & $2.21 \mathrm{E}+3$ & $6.48 \mathrm{E}+2$ \\
\hline \multirow[t]{9}{*}{ UOX } & \multirow[t]{3}{*}{ Actin. } & $\mathbf{K}$ & $1.53 \mathrm{E}+4$ & $2.63 \mathrm{E}+3$ & $6.52 \mathrm{E}+2$ & $3.64 \mathrm{E}+2$ & $3.22 \mathrm{E}+2$ & $1.62 \mathrm{E}+2$ \\
\hline & & $\mathbf{Z}$ & $1.51 \mathrm{E}+4$ & $3.58 \mathrm{E}+3$ & $5.54 \mathrm{E}+2$ & $2.59 \mathrm{E}+2$ & $2.32 \mathrm{E}+2$ & $1.38 \mathrm{E}+2$ \\
\hline & & $\mathbf{L}$ & $1.68 \mathrm{E}+3$ & $1.64 \mathrm{E}+3$ & $5.52 \mathrm{E}+2$ & $2.52 \mathrm{E}+2$ & $2.20 \mathrm{E}+2$ & $1.18 \mathrm{E}+2$ \\
\hline & \multirow[t]{3}{*}{ FP } & $\mathbf{K}$ & $5.26 \mathrm{E}+4$ & $3.74 \mathrm{E}+4$ & $5.37 \mathrm{E}+3$ & $1.82 \mathrm{E}+3$ & $5.25 \mathrm{E}+2$ & $5.35 \mathrm{E}+1$ \\
\hline & & $\mathbf{Z}$ & $6.29 \mathrm{E}+4$ & $4.37 \mathrm{E}+4$ & $6.91 \mathrm{E}+3$ & $2.46 \mathrm{E}+3$ & $6.65 \mathrm{E}+2$ & $6.45 \mathrm{E}+1$ \\
\hline & & $\mathbf{L}$ & $6.58 \mathrm{E}+4$ & $4.58 \mathrm{E}+4$ & $5.92 \mathrm{E}+3$ & $1.99 \mathrm{E}+3$ & $6.88 \mathrm{E}+2$ & $7.36 \mathrm{E}+1$ \\
\hline & \multirow[t]{3}{*}{ SUM } & $\mathbf{K}$ & $6.80 \mathrm{E}+4$ & $4.00 \mathrm{E}+4$ & $6.02 \mathrm{E}+3$ & $2.18 \mathrm{E}+3$ & $8.48 \mathrm{E}+2$ & $2.15 \mathrm{E}+2$ \\
\hline & & $\mathbf{Z}$ & $7.80 \mathrm{E}+4$ & $4.73 E+4$ & $7.46 \mathrm{E}+3$ & $2.72 \mathrm{E}+3$ & $8.97 \mathrm{E}+2$ & $2.02 \mathrm{E}+2$ \\
\hline & & $\mathbf{L}$ & $6.75 \mathrm{E}+4$ & $4.74 \mathrm{E}+4$ & $6.48 \mathrm{E}+3$ & $2.25 \mathrm{E}+3$ & $9.08 \mathrm{E}+2$ & $1.93 \mathrm{E}+2$ \\
\hline
\end{tabular}



APPENDIX E

RESULTS FOR $K_{\text {eff }}$ WITHOUT WATER POOL 



\section{APPENDIX E}

\section{RESULTS FOR $\boldsymbol{K}_{\text {eff }}$ WITHOUT WATER POOL}

For the calculation of the $k_{\text {eff }}$ for the dry ( $0 \mathrm{gm} / \mathrm{cc}$ of water) configuration, KENO-VI calculations were performed with the assemblies touching. That is, the $40 \mathrm{~cm}$ triangular pitch between assemblies was

reduced so that the assemblies were adjacent. The resulting values of $k_{\text {eff }}$ for the three types of fuel were as follows:

$$
\text { UOX - } 0.6636 \text {; MOX-R - } 0.7675 \text {; MOX-W - } 0.7075
$$

Note that these results agree well with some of the Russian data. Note that even though infinite arrays are modelled, the arrays are not infinite in the axial dimension (height is fixed). As the pitch between assemblies increases, the number of neutrons lost to axial leakage increase. 

ORNL/TM-1999/207

\section{INTERNAL DISTRIBUTION}

1. B. B. Bevard

2. S. M. Bowman

3. B. L. Broadhead

4. J. J. Carbajo 5-9. W. C. Carter

10. E. D. Collins

11. B. S. Cowell

12. R. L. Childs

13. M. D. DeHart

14. F. C. Difilippo

15. K. R. Elam

16. R. J. Ellis

17-21. M. B. Emmett

22. I. C. Gauld

23. J. C. Gehin

24. S. R. Greene

25. O. W. Hermann

26. R. Holdaway

27. D. F. Hollenbach

28. T. W. Horning
29. D. T. Ingersoll

30. M. J. Kania

31. M. A. Kuliasha

32. S. Ludwig

33. G. E. Michaels

34. D. L. Moses

35. B. D. Murphy

36-40. C. V. Parks

41. L. M. Petrie

42-46. R. T. Primm III

47. W. J. Reich

48. C. H. Shappert

49. C. C. Southmayd

50. J. C. Wagner

51. R. M. Westfall

52. G. L. Yoder, Jr.

53. Central Research Library

54. ORNL Laboratory Records-RC

\section{EXTERNAL DISTRIBUTION}

55. N. Abdurrahman, College of Engineering, Department of Mechanical Engineering, University of Texas, Austin, TX 78712

56. M. L. Adams, Department of Nuclear Engineering, Texas A\&M University, Zachry 129, College Station, TX 77843

57. D. Alberstein, Los Alamos National Laboratory, MS-E502, P.O. Box 1663, Los Alamos, NM 87545

58. J. Baker, Office of Fissile Materials Disposition, U.S. Department of Energy, MD-3, 1000 Independence Avenue SW, Washington, DC 20585

59. M. S. Chatterton, U.S. Nuclear Regulatory Commission, Office of Nuclear Reactor Regulation, MS O10 B3, Washington, DC 20555-0001

60. K. Chidester, Los Alamos National Laboratory, MS-E502, P.O. Box 1663, Los Alamos, NM 87545 
61. R. H. Clark, Duke Cogema Stone \& Weber, 400 South Tyrone Street, WC-32G, P.O. Box 1004, Charlotte, NC 28202

62. W. Danker, U.S. Department of Energy, MD-3, 1000 Independence Avenue SW, Washington, DC 20585

63. D. Dziadosz, Innsbruck Technical Center, 5000 Dominion Blvd., Glen Allen, VA 23060

64. T. Gould, Lawrence Livermore National Laboratory, P.O. Box 808, MS-L186, Livermore, CA 94551

65. L. Holgate, Office of Fissile Materials Dispostion, U.S. Department of Energy, MD-1/2, 1000 Independence Avenue SW, Washington, DC 20585

66. N. Fletcher, Office of Fissile Materials Dispostion, U.S. Department of Energy, MD-3, 1000 Independence Avenue SW, Washington, DC 20585

67. L. Jardine, Lawrence Livermore National Laboratory, P.O. Box 808, MS-L166, Livermore, CA 94551

68. Dr. Alexander Kalashnikov, Institute of Physics and Power Engineering, 1 Bondarenko Square, Obninsk, Kaluga Region, Russia 249020

69. D. E. Klein, Associate Vice Chancellor for Special Engineering Programs, The University of Texas System, 210 West Sixth Street, Austin, TX 78701

70. S. Nesbit, Duke Cogema Stone \& Weber, 400 South Tyrone Street, WC-32G, P.O. Box 1004, Charlotte, NC 28202

71. J. O. Nulton, Office of Fissile Materials Disposition, U.S. Department of Energy, MD-3, 1000 Independence Avenue SW, Washington, DC 20585

72-73. Office of Scientific and Technical Information, U.S. Department of Energy, P.O. Box 62, Oak Ridge, TN 37831

74. Office of the Assistant Manager for Energy Research and Development, Department of Energy, Oak Ridge Operations (DOE-ORO), P.O. Box 2008, Oak Ridge, TN 37831

75. S. L. Passman, Sandia National Laboratories, Suite 110, 950 L'Enfant Plaza, SW, Washington, DC 20024-2123

76. Dr. Alexander Pavlovitchev, Russian Research Center "Kurchatov Institute," Institute of Nuclear Reactors, VVER Division, VVER Physics Department, 123182, Kurchatov Square, 1, Moscow, Russia

77. K. L. Peddicord, Associate Vice Chancellor, Texas A\&M University, 120 Zachry, College Station, TX 77843-3133

78. W. D. Reece, Texas A\&M University, Department of Nuclear Engineering, Zachry 129, College Station, TX 77843-3133

79. U. Shoop, U.S. Nuclear Regulatory Commission, Office of Nuclear Reactor Regulation, MS O10 B3, Washington, DC 20555-0001 